\title{
Iterative methods for triple hierarchical variational inequalities with mixed equilibrium problems, variational inclusions, and variational inequalities constraints
}

\author{
Lu-Chuan Ceng ${ }^{1}$, Yen-Cherng Lin ${ }^{2 *}$ and Ching-Feng Wen ${ }^{3}$
}

\author{
"Correspondence: \\ yclin@mail.cmu.edu.tw \\ ${ }^{2}$ Department of Occupational \\ Safety and Health, College of Public \\ Health, China Medical University, \\ Taichung, 40421, Taiwan \\ Full list of author information is \\ available at the end of the article
}

\begin{abstract}
In this paper, we introduce and analyze a multi-step hybrid steepest-descent extragradient algorithm and multi-step composite Mann-type viscosity iterative algorithm for finding a solution of triple hierarchical variational inequalities defined over the common set of solutions of mixed equilibrium problems, variational inclusions, variational inequalities, and fixed point problems. Under appropriate assumptions, we prove that the proposed algorithms converge strongly to a common element of the fixed point set of a strict pseudocontractive mapping, a solution set of finitely many generalized mixed equilibrium problems, a solution set of finitely many variational inclusions, and a solution set of a general system of variational inequalities. Such an element is a unique solution of a triple hierarchical variational inequality problem. In addition, we also consider as an application the proposed algorithm to solve a hierarchical variational inequality problem defined over the set of common solutions of finitely many generalized mixed equilibrium problems, finitely many variational inclusions, and a general system of variational inequalities. The results obtained in this paper improve and extend the corresponding results announced by many other authors.

MSC: 49J30; 47H09; 47J20; 49M05
\end{abstract}

Keywords: triple hierarchical variational inequalities; generalized mixed equilibrium problems; variational inclusions; general system of variational inequalities; hybrid steepest-descent extragradient algorithm; composite Mann-type viscosity algorithm; fixed points

\section{Introduction and formulations}

Let $C$ be a nonempty, closed, and convex subset of a real Hilbert space $H$ and $A: C \rightarrow H$ be a nonlinear mapping on $C$. The variational inequality problem (VIP) defined by $C$ and $A$ is to find $x \in C$ such that

$$
\langle A x, y-x\rangle \geq 0, \quad \forall y \in C .
$$

The solution set of VIP (1.1) is denoted by $\operatorname{VI}(C, A)$. The theory of variational inequalities is well established area in nonlinear analysis and optimization. For further details on this topic, we refer to [1-5] and the references therein.

\section{包 Springer}

(c) 2015 Ceng et al.; licensee Springer. This is an Open Access article distributed under the terms of the Creative Commons Attribution License (http://creativecommons.org/licenses/by/4.0), which permits unrestricted use, distribution, and reproduction in any medium, provided the original work is properly credited. 
It is well known that if $A$ is a strongly monotone and Lipschitz-continuous mapping on $C$, then VIP (1.1) has a unique solution. Several iterative methods have been proposed in the literature to compute the approximate solutions of a VIP. Korpelevich?s extragradient method is one of them which was proposed by Korpelevich [6]. During the last two decades, this method received much attention from many authors, who improved and generalized it in various directions and ways; see, for example, [7-18] and the references therein.

Let $\varphi: C \rightarrow \mathbf{R}$ be a real-valued function, $A: C \rightarrow H$ be a nonlinear mapping and $\Theta$ : $C \times C \rightarrow \mathbb{R}$ be a bifunction. The generalized mixed equilibrium problem (GMEP) is to find $x \in C$ such that

$$
\Theta(x, y)+\varphi(y)-\varphi(x)+\langle A x, y-x\rangle \geq 0, \quad \forall y \in C .
$$

We denote the set of solutions of $\operatorname{GMEP}(1.2)$ by $\operatorname{GMEP}(\Theta, \varphi, A)$. The $\operatorname{GMEP}(1.2)$ is very general in the sense that it includes many problems as special cases, namely, optimization problems, variational inequalities, minimax problems, Nash equilibrium problems in noncooperative games, etc. For different aspects and solution methods, we refer to [7, 9, $10,14,18-20]$ and the references therein.

If $\varphi=0$, then GMEP (1.2) reduces to the generalized equilibrium problem (GEP) of finding $x \in C$ such that

$$
\Theta(x, y)+\langle A x, y-x\rangle \geq 0, \quad \forall y \in C .
$$

It was considered and studied in [21,22] and further studied in [23]. The set of solutions of GEP is denoted by $\operatorname{GEP}(\Theta, A)$.

If $A \equiv 0$, then GMEP (1.2) reduces to the mixed equilibrium problem (MEP) which is to find $x \in C$ such that

$$
\Theta(x, y)+\varphi(y)-\varphi(x) \geq 0, \quad \forall y \in C
$$

It was considered and studied in [24]. The set of solutions of MEP is denoted by $\operatorname{MEP}(\Theta, \varphi)$.

If $\varphi \equiv 0, A \equiv 0$, then GMEP reduces to the equilibrium problem (EP) which is to find $x \in C$ such that

$$
\Theta(x, y) \geq 0, \quad \forall y \in C .
$$

The set of solutions of EP is denoted by $\operatorname{EP}(\Theta)$. It is worth to mention that the EP is an unified model of several problems, namely, variational inequality problems, optimization problems, saddle point problems, complementarity problems, fixed point problems, Nash equilibrium problems, etc.

The common assumptions on a bifunction $\Theta: C \times C \rightarrow \mathbb{R}$ are the following:

(A1) $\Theta(x, x)=0$ for all $x \in C$;

(A2) $\Theta$ is monotone, i.e., $\Theta(x, y)+\Theta(y, x) \leq 0$ for any $x, y \in C$;

(A3) $\Theta$ is upper-hemicontinuous, i.e., for each $x, y, z \in C$,

$$
\limsup _{t \rightarrow 0^{+}} \Theta(t z+(1-t) x, y) \leq \Theta(x, y)
$$


(A4) $\Theta(x, \cdot)$ is convex and lower semicontinuous for each $x \in C$.

We also consider the assumptions (B0) and (B1) or (B2) on the function $\varphi: C \rightarrow \mathbb{R}$ :

(B0) $\varphi$ is lower semicontinuous and convex.

(B1) For each $x \in H$ and $r>0$, there exist a bounded subset $D_{x} \subset C$ and $y_{x} \in C$ such that, for any $z \in C \backslash D_{x}$,

$$
\Theta\left(z, y_{x}\right)+\varphi\left(y_{x}\right)-\varphi(z)+\frac{1}{r}\left\langle y_{x}-z, z-x\right\rangle<0 ;
$$

or

(B2) $C$ is a bounded set.

On the other hand, let $B: C \rightarrow H$ be a single-valued mapping and $R$ be a multi-valued mapping with $D(R)=C$. Consider the following variational inclusion: find $x \in C$ such that

$0 \in B x+R x$

We denote by $\mathrm{I}(B, R)$ the solution set of the variational inclusion (1.3). In particular, if $B \equiv R \equiv 0$, then $\mathrm{I}(B, R)=C$. If $B \equiv 0$, then problem (1.3) becomes the inclusion problem introduced by Rockafellar [25]. It is well known that problem (1.3) provides a convenient framework for the unified study of optimal solutions in many optimization related areas including mathematical programming, complementarity problems, variational inequalities, optimal control, mathematical economics, equilibria and game theory, etc. Let a set-valued mapping $R: D(R) \subset H \rightarrow 2^{H}$ be maximal monotone. We define the resolvent operator $J_{R, \lambda}: H \rightarrow \overline{D(R)}$ associated with $R$ and $\lambda$ by

$$
J_{R, \lambda}=(I+\lambda R)^{-1}, \quad \forall x \in H,
$$

where $\lambda$ is a positive number.

Huang [26] studied problem (1.3) in the case where $R$ is maximal monotone and $B$ is strongly monotone and Lipschitz continuous with $D(R)=C=H$. Subsequently, Zeng et al. [27] further studied this problem in a more general setting than in [26]. Moreover, Zeng et al. [27] obtained the same strong convergence result as in [26]. In addition, Zeng et al. [27] also gave the geometric convergence rate estimate for approximate solutions. Also, various types of iterative algorithms for solving variational inclusions have been further studied and developed; for more details, we refer to $[12,28,29]$ and the references therein.

Let $F_{1}, F_{2}: C \rightarrow H$ be two mappings. Consider the general system of variational inequalities (GSVI) of finding $\left(x^{*}, y^{*}\right) \in C \times C$ such that

$$
\begin{cases}\left\langle v_{1} F_{1} y^{*}+x^{*}-y^{*}, x-x^{*}\right\rangle \geq 0, & \forall x \in C, \\ \left\langle v_{2} F_{2} x^{*}+y^{*}-x^{*}, x-y^{*}\right\rangle \geq 0, & \forall x \in C,\end{cases}
$$

where $v_{1}>0$ and $v_{2}>0$ are two constants. It was considered and studied in [11, 15-17, 30]. In particular, if $F_{1} \equiv F_{2} \equiv A$, then the GSVI (1.4) reduces to the problem of finding $\left(x^{*}, y^{*}\right) \in C \times C$ such that

$$
\begin{cases}\left\langle v_{1} A y^{*}+x^{*}-y^{*}, x-x^{*}\right\rangle \geq 0, & \forall x \in C, \\ \left\langle v_{2} A x^{*}+y^{*}-x^{*}, x-y^{*}\right\rangle \geq 0, & \forall x \in C,\end{cases}
$$


which is studied by Verma [31] and is called a new system of variational inequalities (NSVI). Further, if $x^{*}=y^{*}$ additionally, then the NSVI reduces to the classical VIP (1.1). By considering $G:=P_{C}\left(I-v_{1} F_{1}\right) P_{C}\left(I-v_{2} F_{2}\right)$ and $y^{*}=P_{C}\left(I-v_{2} F_{2}\right) x^{*}$, where $P_{C}$ denotes the metric projection of $H$ onto $C$, Ceng et al. [16] transformed GSVI (1.4) into the following fixed point equation:

$$
G x^{*}=x^{*}
$$

A variational inequality problem defined over the set of fixed points of a mapping is called a hierarchical variational inequality problem.

Let $S$ and $T$ be two nonexpansive mappings. Yao et al. [32] considered the following hierarchical variational inequality problem (HVIP): find hierarchically a fixed point of $T$ which is a solution to the VIP for the monotone mapping $I-S$, namely, find $\tilde{x} \in \operatorname{Fix}(T)$ such that

$$
\langle(I-S) \tilde{x}, p-\tilde{x}\rangle \geq 0, \quad \forall p \in \operatorname{Fix}(T)
$$

The solution set of HVIP (1.7) is denoted by $\Lambda$. It is easy to check that solving the HVIP (1.7) is equivalent to solving the fixed point problem of the composite mapping $P_{\mathrm{Fix}(T)} S$, that is, find $\tilde{x} \in C$ such that $\tilde{x}=P_{\mathrm{Fix}(T)} S \tilde{x}$. Ceng et al. [33] introduced and analyzed an iterative algorithm for solving HVIP (1.7). They also studied the strong convergence of the sequences generated by their algorithm.

A variational inequality problem defined over the set of solutions of a hierarchical variational inequality problem is called a triple hierarchical variational inequality problem. For further details of triple hierarchical variational inequalities, we refer to [10,33-36] and the references therein. Very recently, Kong et al. [7] introduced and studied the following triple hierarchical variational inequality problem (THVIP) (over the fixed point set of a strictly pseudocontractive mapping) with a variational inequality constraint.

Problem 1.1 [7, Problem II] Let $F: C \rightarrow H$ be $\kappa$-Lipschitzian and $\eta$-strongly monotone on the nonempty, closed, and convex subset $C$ of $H$, where $\kappa$ and $\eta$ are positive constants. Let $A: C \rightarrow H$ be a monotone and $L$-Lipschitzian mapping, $V: C \rightarrow H$ be a $\rho$-contraction with coefficient $\rho \in[0,1), S: C \rightarrow C$ be a nonexpansive mapping, and $T: C \rightarrow C$ be a $\xi$-strictly pseudocontractive mapping with $\operatorname{Fix}(T) \cap \operatorname{VI}(C, A) \neq \emptyset$. Let $0<\mu<\frac{2 \eta}{\kappa^{2}}$ and $0<$ $\gamma \leq \tau$, where $\tau=1-\sqrt{1-\mu\left(2 \eta-\mu \kappa^{2}\right)}$. Then the objective is to find $x^{*} \in \Xi$ such that

$$
\left\langle(\mu F-\gamma V) x^{*}, x-x^{*}\right\rangle \geq 0, \quad \forall x \in \Xi
$$

where $\Xi$ denotes the solution set of the hierarchical variational inequality problem (HVIP) of finding $z^{*} \in \operatorname{Fix}(T) \cap \operatorname{VI}(C, A)$ such that

$$
\left\langle(\mu F-\gamma S) z^{*}, z-z^{*}\right\rangle \geq 0, \quad \forall z \in \operatorname{Fix}(T) \cap \operatorname{VI}(C, A) .
$$

They proposed an algorithm for solving Problem 1.1 and studied the convergence analysis for the sequences generated by the proposed algorithm.

In this paper, we introduce and study the following triple hierarchical variational inequality problem (THVIP) (defined over the fixed point set of a strictly pseudocontractive 
mapping) with constraints of finitely many GMEPs, finitely many variational inclusions, and a general system of variational inequalities.

Throughout the paper, $M$ and $N$ are assumed to be positive integers.

Problem 1.2 Assume that

(i) for each $j=1,2, F_{j}: C \rightarrow H$ is $\zeta_{j}$-inverse-strongly monotone and $F: H \rightarrow H$ is $\kappa$-Lipschitzian and $\eta$-strongly monotone with positive constants $\kappa, \eta>0$ such that $0<\gamma \leq \tau$ and $0<\mu<\frac{2 \eta}{\kappa^{2}}$ where $\tau=1-\sqrt{1-\mu\left(2 \eta-\mu \kappa^{2}\right)}$;

(ii) for each $k \in\{1,2, \ldots, M\}, \Theta_{k}: C \times C \rightarrow \mathbb{R}$ is a bifunction satisfying conditions (A1)-(A4) and $\varphi_{k}: C \rightarrow \mathbf{R} \cup\{+\infty\}$ is a proper lower semicontinuous and convex function with restriction (B1) or (B2);

(iii) for $k \in\{1,2, \ldots, M\}$ and $i \in\{1,2, \ldots, N\}, R_{i}: C \rightarrow 2^{H}$ is a maximal monotone mapping, and $A_{k}: H \rightarrow H$ and $B_{i}: C \rightarrow H$ are $\mu_{k}$-inverse-strongly monotone and $\eta_{i}$-inverse-strongly monotone, respectively;

(iv) $T: H \rightarrow H$ is a $\xi$-strictly pseudocontractive mapping, $S: H \rightarrow H$ is a nonexpansive mapping and $V: H \rightarrow H$ is a $\rho$-contraction with coefficient $\rho \in[0,1)$;

(v) $\operatorname{VI}(\Omega, \mu F-\gamma S) \neq \emptyset$ where $\Omega:=\bigcap_{k=1}^{M} \operatorname{GMEP}\left(\Theta_{k}, \varphi_{k}, A_{k}\right) \cap \bigcap_{i=1}^{N} \mathrm{I}\left(B_{i}, R_{i}\right) \cap \operatorname{GSVI}(G) \cap \operatorname{Fix}(T)$.

Then the objective is to find $x^{*} \in \Xi$ such that

$$
\left\langle(\mu F-\gamma V) x^{*}, x-x^{*}\right\rangle \geq 0, \quad \forall x \in \Xi
$$

where $\Xi:=\operatorname{VI}(\Omega, \mu F-\gamma S)$, that is, the solution set of the hierarchical variational inequality problem (HVIP) of finding $z^{*} \in \Omega$ such that

$$
\left\langle(\mu F-\gamma S) z^{*}, z-z^{*}\right\rangle \geq 0, \quad \forall z \in \Omega .
$$

Motivated and inspired by the above facts, we introduce and analyze two iterative methods for solving Problem 1.2, that is, THVIP 1.2. By combining Korpelevich?s extragradient method, the viscosity approximation method, the hybrid steepest-descent method, and Mann?s iteration method, we first propose a multi-step hybrid steepest-descent extragradient method. However, by combining Mann?s iteration method, Korpelevich?s extragradient method, the viscosity approximation method, the hybrid steepest-descent method, and the projection method, we propose a multi-step composite Mann-type viscosity iterative algorithm. We prove the strong convergence results for these methods. In particular, we prove that the proposed algorithms converge strongly to a common element $x^{*} \in \Omega:=\bigcap_{k=1}^{M} \operatorname{GMEP}\left(\Theta_{k}, \varphi_{k}, A_{k}\right) \cap \bigcap_{i=1}^{N} \mathrm{I}\left(B_{i}, R_{i}\right) \cap \operatorname{GSVI}(G) \cap \operatorname{Fix}(T)$ which is a unique solution of the THVIP 1.2. In addition, we also consider the application of the proposed algorithm for solving a hierarchical variational inequality problem with constraints of finitely many GMEPs, finitely many variational inclusions and GSVI (1.4). The results obtained in this paper improve and extend the corresponding results announced by many others.

\section{Preliminaries}

Throughout this paper, we assume that $H$ is a real Hilbert space whose inner product and norm are denoted by $\langle\cdot, \cdot \cdot\rangle$ and $\|\cdot\|$, respectively. Let $C$ be a nonempty, closed, and convex subset of $H$. We write $x_{n} \rightarrow x$ to indicate that the sequence $\left\{x_{n}\right\}$ converges weakly to $x$ 
and $x_{n} \rightarrow x$ to indicate that the sequence $\left\{x_{n}\right\}$ converges strongly to $x$. Moreover, we use $\omega_{w}\left(x_{n}\right)$ to denote the weak $\omega$-limit set of the sequence $\left\{x_{n}\right\}$, that is,

$$
\omega_{w}\left(x_{n}\right):=\left\{x \in H: x_{n_{i}} \rightarrow x \text { for some subsequence }\left\{x_{n_{i}}\right\} \text { of }\left\{x_{n}\right\}\right\} .
$$

Definition 2.1 A mapping $A: C \rightarrow H$ is called

(i) monotone if

$$
\langle A x-A y, x-y\rangle \geq 0, \quad \forall x, y \in C ;
$$

(ii) $\eta$-strongly monotone if there exists a constant $\eta>0$ such that

$$
\langle A x-A y, x-y\rangle \geq \eta\|x-y\|^{2}, \quad \forall x, y \in C ;
$$

(iii) $\zeta$-inverse-strongly monotone if there exists a constant $\zeta>0$ such that

$$
\langle A x-A y, x-y\rangle \geq \zeta\|A x-A y\|^{2}, \quad \forall x, y \in C .
$$

It is easy to see that the projection $P_{C}$ is 1-inverse-strongly monotone. Inverse-strongly monotone (also referred to as co-coercive) operators have been applied widely in solving practical problems in various fields. It is obvious that if $A$ is $\zeta$-inverse-strongly monotone, then $A$ is monotone and $\frac{1}{\zeta}$-Lipschitz continuous. Moreover, we also have for all $u, v \in C$ and $\lambda>0$,

$$
\begin{aligned}
\|(I-\lambda A) u-(I-\lambda A) v\|^{2} & =\|(u-v)-\lambda(A u-A v)\|^{2} \\
& =\|u-v\|^{2}-2 \lambda\langle A u-A v, u-v\rangle+\lambda^{2}\|A u-A v\|^{2} \\
& \leq\|u-v\|^{2}+\lambda(\lambda-2 \zeta)\|A u-A v\|^{2} .
\end{aligned}
$$

So, if $\lambda \leq 2 \zeta$, then $I-\lambda A$ is a nonexpansive mapping from $C$ to $H$.

The metric (or nearest point) projection from $H$ onto $C$ is the mapping $P_{C}: H \rightarrow C$ which assigns to each point $x \in H$ the unique point $P_{C} x \in C$ satisfying the property

$$
\left\|x-P_{C} x\right\|=\inf _{y \in C}\|x-y\|=: d(x, C)
$$

Some important properties of projections are gathered in the following proposition.

Proposition 2.1 For given $x \in H$ and $z \in C$ :

(i) $z=P_{C} x \Leftrightarrow\langle x-z, y-z\rangle \leq 0, \forall y \in C$;

(ii) $z=P_{C} x \Leftrightarrow\|x-z\|^{2} \leq\|x-y\|^{2}-\|y-z\|^{2}, \forall y \in C$;

(iii) $\left\langle P_{C} x-P_{C} y, x-y\right\rangle \geq\left\|P_{C} x-P_{C} y\right\|^{2}, \forall y \in H$. Consequently, $P_{C}$ is nonexpansive and monotone.

Definition 2.2 A mapping $T: H \rightarrow H$ is said to be

(a) nonexpansive if

$$
\|T x-T y\| \leq\|x-y\|, \quad \forall x, y \in H
$$


(b) firmly nonexpansive if $2 T-I$ is nonexpansive, or equivalently, if $T$ is 1 -inverse-strongly monotone (1-ism),

$$
\langle x-y, T x-T y\rangle \geq\|T x-T y\|^{2}, \quad \forall x, y \in H
$$

alternatively, $T$ is firmly nonexpansive if and only if $T$ can be expressed as

$$
T=\frac{1}{2}(I+S)
$$

where $S: H \rightarrow H$ is nonexpansive; projections are firmly nonexpansive.

It can easily be seen that if $T$ is nonexpansive, then $I-T$ is monotone.

Next we list some elementary conclusions for the MEP.

Proposition 2.2 [24] Assume that $\Theta: C \times C \rightarrow \mathbb{R}$ satisfies (A1)-(A4) and let $\varphi: C \rightarrow \mathbb{R}$ be a proper lower semicontinuous and convex function. Assume that either (B1) or (B2) holds. For $r>0$ and $x \in H$, define a mapping $T_{r}^{(\Theta, \varphi)}: H \rightarrow C$ as follows:

$$
T_{r}^{(\Theta, \varphi)}(x):=\left\{z \in C: \Theta(z, y)+\varphi(y)-\varphi(z)+\frac{1}{r}\langle y-z, z-x\rangle \geq 0, \forall y \in C\right\}
$$

for all $x \in H$. Then the following statements hold:

(i) For each $x \in H, T_{r}^{(\Theta, \varphi)}(x)$ is nonempty and single-valued;

(ii) $T_{r}^{(\Theta, \varphi)}$ is firmly nonexpansive, that is, for any $x, y \in H$,

$$
\left\|T_{r}^{(\Theta, \varphi)} x-T_{r}^{(\Theta, \varphi)} y\right\|^{2} \leq\left\langle T_{r}^{(\Theta, \varphi)} x-T_{r}^{(\Theta, \varphi)} y, x-y\right\rangle
$$

(iii) $\operatorname{Fix}\left(T_{r}^{(\Theta, \varphi)}\right)=\operatorname{MEP}(\Theta, \varphi)$;

(iv) $\operatorname{MEP}(\Theta, \varphi)$ is closed and convex;

(v) $\left\|T_{s}^{(\Theta, \varphi)} x-T_{t}^{(\Theta, \varphi)} x\right\|^{2} \leq \frac{s-t}{s}\left\langle T_{s}^{(\Theta, \varphi)} x-T_{t}^{(\Theta, \varphi)} x, T_{s}^{(\Theta, \varphi)} x-x\right\rangle$ for all $s, t>0$ and $x \in H$.

Ceng et al. [16] transformed the GSVI (1.4) into a fixed point problem in the following way.

Proposition 2.3 [16] For given $\bar{x}, \bar{y} \in C,(\bar{x}, \bar{y})$ is a solution of the GSVI (1.4) if and only if $\bar{x}$ is a fixed point of the mapping $G: C \rightarrow C$ defined by

$$
G x=P_{C}\left(I-v_{1} F_{1}\right) P_{C}\left(I-v_{2} F_{2}\right) x, \quad \forall x \in C,
$$

where $\bar{y}=P_{C}\left(I-v_{2} F_{2}\right) \bar{x}$.

In particular, if the mapping $F_{j}: C \rightarrow H$ is $\zeta_{j}$-inverse-strongly monotone for $j=1,2$, then the mapping $G$ is nonexpansive provided $v_{j} \in\left(0,2 \zeta_{j}\right]$ for $j=1,2$. We denote by $\operatorname{GSVI}(G)$ the fixed point set of the mapping $G$.

We need some facts and tools in a real Hilbert space $H$ which are listed as lemmas below.

Lemma 2.1 Let $X$ be a real inner product space. Then we have the following inequality:

$$
\|x+y\|^{2} \leq\|x\|^{2}+2\langle y, x+y\rangle, \quad \forall x, y \in X
$$


Lemma 2.2 Let $H$ be a real Hilbert space. Then the following hold:

(a) $\|x-y\|^{2}=\|x\|^{2}-\|y\|^{2}-2\langle x-y, y\rangle$ for all $x, y \in H$.

(b) $\|\lambda x+\mu y\|^{2}=\lambda\|x\|^{2}+\mu\|y\|^{2}-\lambda \mu\|x-y\|^{2}$ for all $x, y \in H$ and $\lambda, \mu \in[0,1]$ with $\lambda+\mu=1$.

(c) If $\left\{x_{n}\right\}$ is a sequence in $H$ such that $x_{n} \rightarrow x$, it follows that

$$
\limsup _{n \rightarrow \infty}\left\|x_{n}-y\right\|^{2}=\limsup _{n \rightarrow \infty}\left\|x_{n}-x\right\|^{2}+\|x-y\|^{2}, \quad \forall y \in H .
$$

It is clear that, in a real Hilbert space $H, T: C \rightarrow C$ is $\xi$-strictly pseudocontractive if and only if the following inequality holds:

$$
\langle T x-T y, x-y\rangle \leq\|x-y\|^{2}-\frac{1-\xi}{2}\|(I-T) x-(I-T) y\|^{2}, \quad \forall x, y \in C .
$$

This immediately implies that if $T$ is a $\xi$-strictly pseudocontractive mapping, then $I-T$ is $\frac{1-\xi}{2}$-inverse-strongly monotone; for further details, we refer to [37] and the references therein. It is well known that the class of strict pseudocontractions strictly includes the class of nonexpansive mappings and that the class of pseudocontractions strictly includes the class of strict pseudocontractions.

Lemma 2.3 [37, Proposition 2.1] Let C be a nonempty, closed, and convex subset of a real Hilbert space $H$ and $T: C \rightarrow C$ be a mapping.

(i) If $T$ is a $\xi$-strictly pseudocontractive mapping, then $T$ satisfies the Lipschitzian condition

$$
\|T x-T y\| \leq \frac{1+\xi}{1-\xi}\|x-y\|, \quad \forall x, y \in C .
$$

(ii) If $T$ is a $\xi$-strictly pseudocontractive mapping, then the mapping $I-T$ is semiclosed at 0 , that is, if $\left\{x_{n}\right\}$ is a sequence in $C$ such that $x_{n} \rightarrow \tilde{x}$ and $(I-T) x_{n} \rightarrow 0$, then $(I-T) \tilde{x}=0$.

(iii) If $T$ is $\xi$-(quasi-)strict pseudocontraction, then the fixed-point set $\operatorname{Fix}(T)$ of $T$ is closed and convex so that the projection $P_{\mathrm{Fix}(T)}$ is well defined.

Lemma 2.4 [11] Let C be a nonempty, closed, and convex subset of a real Hilbert space $H$. Let $T: C \rightarrow C$ be a $\xi$-strictly pseudocontractive mapping. Let $\gamma$ and $\delta$ be two nonnegative real numbers such that $(\gamma+\delta) \xi \leq \gamma$. Then

$$
\|\gamma(x-y)+\delta(T x-T y)\| \leq(\gamma+\delta)\|x-y\|, \quad \forall x, y \in C .
$$

Lemma 2.5 (Demiclosedness principle [38]) Let C be a nonempty, closed, and convex subset of a real Hilbert space $H$. Let $S$ be a nonexpansive self-mapping on $C$ with $\operatorname{Fix}(S) \neq \emptyset$. Then $I-S$ is demiclosed. That is, whenever $\left\{x_{n}\right\}$ is a sequence in $C$ weakly converging to some $x \in C$ and the sequence $\left\{(I-S) x_{n}\right\}$ strongly converges to some $y$, it follows that $(I-S) x=y$. Here $I$ is the identity operator of $H$.

Lemma 2.6 Let $A: C \rightarrow H$ be a monotone mapping. In the context of the variational inequality problem the characterization of the projection (see Proposition 2.1(i)) implies

$$
u \in \operatorname{VI}(C, A) \quad \Leftrightarrow \quad u=P_{C}(u-\lambda A u), \quad \forall \lambda>0 .
$$


Let $C$ be a nonempty, closed, and convex subset of a real Hilbert space $H$. Let $\lambda$ be a number in $(0,1]$ and let $\mu>0$. Associated with a nonexpansive mapping $T: C \rightarrow H$, we define the mapping $T^{\lambda}: C \rightarrow H$ by

$$
T^{\lambda} x:=T x-\lambda \mu F(T x), \quad \forall x \in C,
$$

where $F: H \rightarrow H$ is an operator such that, for some positive constants $\kappa, \eta>0, F$ is $\kappa$-Lipschitzian and $\eta$-strongly monotone on $H$; that is, $F$ satisfies the conditions:

$$
\|F x-F y\| \leq \kappa\|x-y\| \quad \text { and } \quad\langle F x-F y, x-y\rangle \geq \eta\|x-y\|^{2}
$$

for all $x, y \in H$.

Lemma 2.7 [39, Lemma 3.1] $T^{\lambda}$ is a contraction provided $0<\mu<\frac{2 \eta}{\kappa^{2}}$; that is,

$$
\left\|T^{\lambda} x-T^{\lambda} y\right\| \leq(1-\lambda \tau)\|x-y\|, \quad \forall x, y \in C,
$$

where $\tau=1-\sqrt{1-\mu\left(2 \eta-\mu \kappa^{2}\right)} \in(0,1]$.

\section{Remark 2.1}

(i) Since $F$ is $\kappa$-Lipschitzian and $\eta$-strongly monotone on $H$, we get $0<\eta \leq \kappa$. Hence, whenever $0<\mu<\frac{2 \eta}{\kappa^{2}}$, we have

$$
\begin{aligned}
0 & \leq(1-\mu \eta)^{2}=1-2 \mu \eta+\mu^{2} \eta^{2} \\
& \leq 1-2 \mu \eta+\mu^{2} \kappa^{2} \\
& <1-2 \mu \eta+\frac{2 \eta}{\kappa^{2}} \mu \kappa^{2}=1,
\end{aligned}
$$

which implies

$$
0<1-\sqrt{1-2 \mu \eta+\mu^{2} \kappa^{2}} \leq 1 .
$$

So, $\tau=1-\sqrt{1-\mu\left(2 \eta-\mu \kappa^{2}\right)} \in(0,1]$.

(ii) In Lemma 2.7, put $F=\frac{1}{2} I$ and $\mu=2$. Then we know that $\kappa=\eta=\frac{1}{2}$,

$0<\mu=2<\frac{2 \eta}{\kappa^{2}}=4$, and

$$
\tau=1-\sqrt{1-\mu\left(2 \eta-\mu \kappa^{2}\right)}=1-\sqrt{1-2\left(2 \times \frac{1}{2}-2 \times\left(\frac{1}{2}\right)^{2}\right)}=1 .
$$

Lemma 2.8 [39] Let $\left\{s_{n}\right\}$ be a sequence of nonnegative numbers satisfying the conditions

$$
s_{n+1} \leq\left(1-\alpha_{n}\right) s_{n}+\alpha_{n} \beta_{n}, \quad \forall n \geq 1,
$$

where $\left\{\alpha_{n}\right\}$ and $\left\{\beta_{n}\right\}$ are sequences of real numbers such that

(i) $\left\{\alpha_{n}\right\} \subset[0,1]$ and $\sum_{n=1}^{\infty} \alpha_{n}=\infty$, or equivalently,

$$
\prod_{n=1}^{\infty}\left(1-\alpha_{n}\right):=\lim _{n \rightarrow \infty} \prod_{k=1}^{n}\left(1-\alpha_{k}\right)=0 ;
$$


(ii) $\lim \sup _{n \rightarrow \infty} \beta_{n} \leq 0$, or $\sum_{n=1}^{\infty}\left|\alpha_{n} \beta_{n}\right|<\infty$.

Then $\lim _{n \rightarrow \infty} s_{n}=0$.

Finally, recall that a set-valued mapping $T: D(T) \subset H \rightarrow 2^{H}$ is called monotone if for all $x, y \in D(T), f \in T x$ and $g \in T y$ imply

$$
\langle f-g, x-y\rangle \geq 0
$$

A set-valued mapping $T$ is called maximal monotone if $T$ is monotone and $(I+\lambda T) D(T)=$ $H$ for each $\lambda>0$, where $I$ is the identity mapping of $H$. We denote by $G(T)$ the graph of $T$. It is well known that a monotone mapping $T$ is maximal if and only if, for $(x, f) \in H \times H$, $\langle f-g, x-y\rangle \geq 0$ for every $(y, g) \in G(T)$ implies $f \in T x$.

Next we provide an example to illustrate the concept of maximal monotone mapping.

Let $A: C \rightarrow H$ be a monotone, $k$-Lipschitz-continuous mapping and let $N_{C} v$ be the normal cone to $C$ at $v \in C$, that is,

$$
N_{C} v=\{u \in H:\langle v-p, u\rangle \geq 0, \forall p \in C\}
$$

Define

$$
\widetilde{T} v= \begin{cases}A v+N_{C} v, & \text { if } v \in C, \\ \emptyset, & \text { if } v \notin C .\end{cases}
$$

Then $\widetilde{T}$ is maximal monotone (see [25]) such that

$$
0 \in \widetilde{T} v \quad \Leftrightarrow \quad v \in \operatorname{VI}(C, A) .
$$

Let $R: D(R) \subset H \rightarrow 2^{H}$ be a maximal monotone mapping. Let $\lambda, \mu>0$ be two positive numbers.

Lemma 2.9 [40] We have the resolvent identity

$$
J_{R, \lambda} x=J_{R, \mu}\left(\frac{\mu}{\lambda} x+\left(1-\frac{\mu}{\lambda}\right) J_{R, \lambda} x\right), \quad \forall x \in H
$$

Remark 2.2 For $\lambda, \mu>0$, we have the following relation:

$$
\left\|J_{R, \lambda} x-J_{R, \mu} y\right\| \leq\|x-y\|+|\lambda-\mu|\left(\frac{1}{\lambda}\left\|J_{R, \lambda} x-y\right\|+\frac{1}{\mu}\left\|x-J_{R, \mu} y\right\|\right), \quad \forall x, y \in H .
$$

Indeed, whenever $\lambda \geq \mu$, utilizing Lemma 2.9 we deduce that

$$
\begin{aligned}
\left\|J_{R, \lambda} x-J_{R, \mu} y\right\| & =\left\|J_{R, \mu}\left(\frac{\mu}{\lambda} x+\left(1-\frac{\mu}{\lambda}\right) J_{R, \lambda} x\right)-J_{R, \mu} y\right\| \\
& \leq\left\|\frac{\mu}{\lambda} x+\left(1-\frac{\mu}{\lambda}\right) J_{R, \lambda} x-y\right\| \\
& \leq \frac{\mu}{\lambda}\|x-y\|+\left(1-\frac{\mu}{\lambda}\right)\left\|J_{R, \lambda} x-y\right\| \\
& \leq\|x-y\|+\frac{|\lambda-\mu|}{\lambda}\left\|J_{R, \lambda} x-y\right\| .
\end{aligned}
$$


Similarly, whenever $\lambda<\mu$, we get

$$
\left\|J_{R, \lambda} x-J_{R, \mu} y\right\| \leq\|x-y\|+\frac{|\lambda-\mu|}{\mu}\left\|x-J_{R, \mu} y\right\| .
$$

Combining the above two cases we conclude that (2.3) holds.

In terms of Huang [26] (see also [27]), we have the following property for the resolvent operator $J_{R, \lambda}: H \rightarrow \overline{D(R)}$.

Lemma 2.10 $J_{R, \lambda}$ is single-valued and firmly nonexpansive, that is,

$$
\left\langle J_{R, \lambda} x-J_{R, \lambda} y, x-y\right\rangle \geq\left\|J_{R, \lambda} x-J_{R, \lambda} y\right\|^{2}, \quad \forall x, y \in H .
$$

Consequently, $J_{R, \lambda}$ is nonexpansive and monotone.

Lemma 2.11 [12] Let $R$ be a maximal monotone mapping with $D(R)=C$. Then for any given $\lambda>0, u \in C$ is a solution of problem (1.5) if and only if $u \in C$ satisfies

$$
u=J_{R, \lambda}(u-\lambda B u) .
$$

Lemma 2.12 [27] Let $R$ be a maximal monotone mapping with $D(R)=C$ and let $B: C \rightarrow H$ be a strongly monotone, continuous, and single-valued mapping. Then for each $z \in H$, the equation $z \in(B+\lambda R) x$ has a unique solution $x_{\lambda}$ for $\lambda>0$.

Lemma 2.13 [12] Let $R$ be a maximal monotone mapping with $D(R)=C$ and $B: C \rightarrow H$ be a monotone, continuous, and single-valued mapping. Then $(I+\lambda(R+B)) C=H$ for each $\lambda>0$. In this case, $R+B$ is maximal monotone.

\section{Hybrid steepest-descent extragradient method and convergence results}

In this section, we introduce and analyze a multi-step hybrid steepest-descent extragradient algorithm for finding a solution of THVIP 1.2. This algorithm is based on Korpelevich?s extragradient method, the viscosity approximation method, the hybrid steepest-descent method, and Mann?s iteration method. We prove the strong convergence of the proposed algorithm to a unique solution of THVIP 1.2 under suitable conditions. In addition, we also consider the application of the proposed algorithm for solving a hierarchical variational inequality problem (HVIP).

In the rest of the paper, unless otherwise specified, we assume that $M$ and $N$ are positive integers, and $C$ is a nonempty, closed, and convex subset of a real Hilbert space $H$.

\section{Assumption 3.1}

(a) For each $k \in\{1,2, \ldots, M\}$, let $\Theta_{k}: C \times C \rightarrow \mathbb{R}$ be a bifunction satisfying (A1)-(A4) and $\varphi_{k}: C \rightarrow \mathbb{R} \cup\{+\infty\}$ be a proper lower semicontinuous and convex function with restriction (B1) or (B2).

(b) For each $k \in\{1,2, \ldots, M\}$ and each $i \in\{1,2, \ldots, N\}$, let $R_{i}: C \rightarrow 2^{H}$ be a maximal monotone mapping, and $A_{k}: H \rightarrow H$ and $B_{i}: C \rightarrow H$ be $\mu_{k}$-inverse-strongly monotone and $\eta_{i}$-inverse-strongly monotone, respectively. 
(c) Let $T: H \rightarrow H$ be a $\xi$-strictly pseudocontractive mapping, $S: H \rightarrow H$ be a nonexpansive mapping and $V: H \rightarrow H$ be a $\rho$-contraction with coefficient $\rho \in[0,1)$.

(d) For $j=1,2$, let $F_{j}: C \rightarrow H$ be $\zeta_{j}$-inverse-strongly monotone, and $F: H \rightarrow H$ be $\kappa$-Lipschitzian and $\eta$-strongly monotone with positive constants $\kappa, \eta>0$ such that $0<\mu<\frac{2 \eta}{\kappa^{2}}$ and $0<\gamma \leq \tau$, where $\tau=1-\sqrt{1-\mu\left(2 \eta-\mu \kappa^{2}\right)}$.

(e) $\Omega:=\bigcap_{k=1}^{M} \operatorname{GMEP}\left(\Theta_{k}, \varphi_{k}, A_{k}\right) \cap \bigcap_{i=1}^{N} \mathrm{I}\left(B_{i}, R_{i}\right) \cap \operatorname{GSVI}(G) \cap \operatorname{Fix}(T)$.

(f) The solution set $\Xi$ of HVIP (1.11) is nonempty.

(g) Let $\left\{\alpha_{n}\right\},\left\{\lambda_{n}\right\} \subset(0,1],\left\{\beta_{n}\right\},\left\{\gamma_{n}\right\},\left\{\delta_{n}\right\} \subset[0,1],\left\{\lambda_{i, n}\right\} \subset\left[a_{i}, b_{i}\right] \subset\left(0,2 \eta_{i}\right)$, and $\left\{r_{k, n}\right\} \subset\left[c_{k}, d_{k}\right] \subset\left(0,2 \mu_{k}\right)$, where $i \in\{1,2, \ldots, N\}$ and $k \in\{1,2, \ldots, M\}$.

We propose the following multi-step hybrid steepest-descent extragradient algorithm for finding a solution of THVIP 1.2.

Algorithm 3.1 For given arbitrarily $x_{0} \in H$, let $\left\{x_{n}\right\}$ be the sequence generated by

$$
\left\{\begin{array}{l}
u_{n}=T_{r_{M, n}}^{\left(\Theta_{M}, \varphi_{M}\right)}\left(I-r_{M, n} A_{M}\right) T_{r_{M-1, n}}^{\left(\Theta_{M-1}, \varphi_{M-1}\right)}\left(I-r_{M-1, n} A_{M-1}\right) \cdots T_{\left.r_{1, n}, \varphi_{1}\right)}^{\left(\Theta_{1}\right.}\left(I-r_{1, n} A_{1}\right) x_{n}, \\
v_{n}=J_{R_{N}, \lambda_{N, n}}\left(I-\lambda_{N, n} B_{N}\right) J_{R_{N-1}, \lambda_{N-1, n}}\left(I-\lambda_{N-1, n} B_{N-1}\right) \cdots J_{R_{1}, \lambda_{1, n}}\left(I-\lambda_{1, n} B_{1}\right) u_{n}, \\
y_{n}=\beta_{n} x_{n}+\gamma_{n} G v_{n}+\delta_{n} T G v_{n}, \\
x_{n+1}=\lambda_{n} \gamma\left(\alpha_{n} V x_{n}+\left(1-\alpha_{n}\right) S x_{n}\right)+\left(I-\lambda_{n} \mu F\right) y_{n}, \quad \forall n \geq 0,
\end{array}\right.
$$

where $G:=P_{C}\left(I-v_{1} F_{1}\right) P_{C}\left(I-v_{2} F_{2}\right)$ with $v_{j} \in\left(0,2 \zeta_{j}\right)$ for $j=1,2$.

If $V \equiv 0$, then Algorithm 3.1 reduces to the following algorithm.

Algorithm 3.2 For given arbitrarily $x_{0} \in H$, let $\left\{x_{n}\right\}$ be the sequence generated by

$$
\left\{\begin{array}{l}
u_{n}=T_{r_{M, n}}^{\left(\Theta_{M}, \varphi_{M}\right)}\left(I-r_{M, n} A_{M}\right) T_{r_{M-1, n}}^{\left(\Theta_{M-1}, \varphi_{M-1}\right)}\left(I-r_{M-1, n} A_{M-1}\right) \cdots T_{\left.r_{1, n}, \varphi_{1}\right)}^{\left(\Theta_{1}\right.}\left(I-r_{1, n} A_{1}\right) x_{n}, \\
v_{n}=J_{R_{N}, \lambda_{N, n}}\left(I-\lambda_{N, n} B_{N}\right) J_{R_{N-1}, \lambda_{N-1, n}}\left(I-\lambda_{N-1, n} B_{N-1}\right) \cdots J_{R_{1}, \lambda_{1, n}}\left(I-\lambda_{1, n} B_{1}\right) u_{n}, \\
y_{n}=\beta_{n} x_{n}+\gamma_{n} G v_{n}+\delta_{n} T G v_{n}, \\
x_{n+1}=\lambda_{n}\left(1-\alpha_{n}\right) \gamma S x_{n}+\left(I-\lambda_{n} \mu F\right) y_{n}, \quad \forall n \geq 0 .
\end{array}\right.
$$

The following result provides the convergence of the sequences generated by the above algorithm.

Theorem 3.1 In addition to Assumption 3.1, suppose that

(i) $\lim _{n \rightarrow \infty} \lambda_{n}=0, \sum_{n=0}^{\infty} \lambda_{n} \alpha_{n}=\infty$ and $\lim _{n \rightarrow \infty} \frac{1}{\lambda_{n} \alpha_{n}}\left|1-\frac{\alpha_{n-1}}{\alpha_{n}}\right|=0$;

(ii) $\limsup _{n \rightarrow \infty} \frac{\alpha_{n}}{\lambda_{n}}<\infty, \lim _{n \rightarrow \infty} \frac{1}{\lambda_{n} \alpha_{n}}\left|\frac{1}{\alpha_{n}}-\frac{1}{\alpha_{n-1}}\right|=0$ and $\lim _{n \rightarrow \infty} \frac{1}{\alpha_{n}^{2}}\left|1-\frac{\lambda_{n-1}}{\lambda_{n}}\right|=0$;

(iii) $\lim _{n \rightarrow \infty} \frac{\left|\beta_{n}-\beta_{n-1}\right|}{\lambda_{n} \alpha_{n}^{2}}=0$ and $\lim _{n \rightarrow \infty} \frac{\left|\gamma_{n}-\gamma_{n-1}\right|}{\lambda_{n} \alpha_{n}^{2}}=0$;

(iv) $\lim _{n \rightarrow \infty} \frac{\left|\lambda_{i, n}-\lambda_{i, n-1}\right|}{\lambda_{n} \alpha_{n}^{2}}=0$ and $\lim _{n \rightarrow \infty} \frac{\left|r_{k, n}-r_{k, n-1}\right|}{\lambda_{n} \alpha_{n}^{2}}=0$ for $i=1,2, \ldots, N$ and $k=1,2, \ldots, M$

(v) $\beta_{n}+\gamma_{n}+\delta_{n}=1,\left(\gamma_{n}+\delta_{n}\right) \xi \leq \gamma_{n}(\forall n \geq 0),\left\{\beta_{n}\right\} \subset[a, b] \subset(0,1)$, and $\liminf _{n \rightarrow \infty} \delta_{n}>0$;

(vi) $\|x-T x\| \geq \bar{k}[d(x, \Omega)]^{\theta}(\forall x \in C)$ and $\lim _{n \rightarrow \infty}\left(\lambda_{n}^{1 / \theta} / \alpha_{n}\right)=0$ for some $\bar{k}, \theta>0$.

Then the following conclusions hold.

(a) If $\left\{S x_{n}\right\}$ is bounded, then $\left\{x_{n}\right\}$ converges strongly to a point $x^{*} \in \Omega$ which is a unique solution of Problem 1.2 provided that $\left\|y_{n}-T x_{n}\right\|=o\left(\alpha_{n}\right)$. 
(b) If $\left\{S x_{n}\right\}$ is bounded, where $\left\{x_{n}\right\}$ is the sequence generated by (3.2), then $\left\{x_{n}\right\}$ converges strongly to a unique solution $x^{*} \in \Xi$ of the following VIP provided that $\left\|y_{n}-T x_{n}\right\|=o\left(\alpha_{n}\right)$ :

$$
\left\langle F x^{*}, x-x^{*}\right\rangle \geq 0, \quad \forall x \in \Xi
$$

Proof Put

$$
\Delta_{n}^{k}=T_{r_{k, n}}^{\left(\Theta_{k}, \varphi_{k}\right)}\left(I-r_{k, n} A_{k}\right) T_{r_{k-1, n}}^{\left(\Theta_{k-1}, \varphi_{k-1}\right)}\left(I-r_{k-1, n} A_{k-1}\right) \cdots T_{r_{1, n}}^{\left(\Theta_{1}, \varphi_{1}\right)}\left(I-r_{1, n} A_{1}\right) x_{n}
$$

for all $k \in\{1,2, \ldots, M\}$ and $n \geq 1$,

$$
\Lambda_{n}^{i}=J_{R_{i}, \lambda_{i, n}}\left(I-\lambda_{i, n} B_{i}\right) J_{R_{i-1}, \lambda_{i-1, n}}\left(I-\lambda_{i-1, n} B_{i-1}\right) \cdots J_{R_{1}, \lambda_{1, n}}\left(I-\lambda_{1, n} B_{1}\right)
$$

for all $i \in\{1,2, \ldots, N\}, \Delta_{n}^{0}=I$, and $\Lambda_{n}^{0}=I$, where $I$ is the identity mapping on $H$. Then we have $u_{n}=\Delta_{n}^{M} x_{n}$ and $v_{n}=\Lambda_{n}^{N} u_{n}$.

We divide the rest of the proof into several steps.

Step 1 . We prove that $\left\{x_{n}\right\}$ is bounded.

Indeed, take a fixed $p \in \Omega$ arbitrarily. Utilizing (2.1) and Proposition 2.2(ii), we have

$$
\begin{aligned}
\left\|u_{n}-p\right\|= & \left\|T_{r_{M, n}}^{\left(\Theta_{M}, \varphi_{M}\right)}\left(I-r_{M, n} B_{M}\right) \Delta_{n}^{M-1} x_{n}-T_{r_{M, n}}^{\left(\Theta_{M}, \varphi_{M}\right)}\left(I-r_{M, n} B_{M}\right) \Delta_{n}^{M-1} p\right\| \\
\leq & \left\|\left(I-r_{M, n} B_{M}\right) \Delta_{n}^{M-1} x_{n}-\left(I-r_{M, n} B_{M}\right) \Delta_{n}^{M-1} p\right\| \\
\leq & \left\|\Delta_{n}^{M-1} x_{n}-\Delta_{n}^{M-1} p\right\| \\
& \vdots \\
\leq & \left\|\Delta_{n}^{0} x_{n}-\Delta_{n}^{0} p\right\| \\
= & \left\|x_{n}-p\right\| .
\end{aligned}
$$

Utilizing (2.1) and Lemma 2.10, we have

$$
\begin{aligned}
\left\|v_{n}-p\right\|= & \left\|J_{R_{N}, \lambda_{N, n}}\left(I-\lambda_{N, n} A_{N}\right) \Lambda_{n}^{N-1} u_{n}-J_{R_{N}, \lambda_{N, n}}\left(I-\lambda_{N, n} A_{N}\right) \Lambda_{n}^{N-1} p\right\| \\
\leq & \left\|\left(I-\lambda_{N, n} A_{N}\right) \Lambda_{n}^{N-1} u_{n}-\left(I-\lambda_{N, n} A_{N}\right) \Lambda_{n}^{N-1} p\right\| \\
\leq & \left\|\Lambda_{n}^{N-1} u_{n}-\Lambda_{n}^{N-1} p\right\| \\
& \vdots \\
\leq & \left\|\Lambda_{n}^{0} u_{n}-\Lambda_{n}^{0} p\right\| \\
= & \left\|u_{n}-p\right\|
\end{aligned}
$$

which together with the last inequality implies that

$$
\left\|v_{n}-p\right\| \leq\left\|x_{n}-p\right\|
$$


Since $p=G p=P_{C}\left(I-v_{1} F_{1}\right) P_{C}\left(I-v_{2} F_{2}\right) p, F_{j}$ is $\zeta_{j}$-inverse-strongly monotone for $j=1,2$, and $0<v_{j} \leq 2 \zeta_{j}$ for $j=1,2$, we deduce that, for any $n \geq 0$,

$$
\begin{aligned}
&\left\|G v_{n}-p\right\|^{2} \\
&=\left\|P_{C}\left(I-v_{1} F_{1}\right) P_{C}\left(I-v_{2} F_{2}\right) v_{n}-P_{C}\left(I-v_{1} F_{1}\right) P_{C}\left(I-v_{2} F_{2}\right) p\right\|^{2} \\
& \leq\left\|\left(I-v_{1} F_{1}\right) P_{C}\left(I-v_{2} F_{2}\right) v_{n}-\left(I-v_{1} F_{1}\right) P_{C}\left(I-v_{2} F_{2}\right) p\right\|^{2} \\
&=\left\|\left[P_{C}\left(I-v_{2} F_{2}\right) v_{n}-P_{C}\left(I-v_{2} F_{2}\right) p\right]-v_{1}\left[F_{1} P_{C}\left(I-v_{2} F_{2}\right) v_{n}-F_{1} P_{C}\left(I-v_{2} F_{2}\right) p\right]\right\|^{2} \\
& \leq\left\|P_{C}\left(I-v_{2} F_{2}\right) v_{n}-P_{C}\left(I-v_{2} F_{2}\right) p\right\|^{2} \\
&+v_{1}\left(v_{1}-2 \zeta_{1}\right)\left\|F_{1} P_{C}\left(I-v_{2} F_{2}\right) v_{n}-F_{1} P_{C}\left(I-v_{2} F_{2}\right) p\right\|^{2} \\
& \leq\left\|P_{C}\left(I-v_{2} F_{2}\right) v_{n}-P_{C}\left(I-v_{2} F_{2}\right) p\right\|^{2} \\
& \leq\left\|\left(I-v_{2} F_{2}\right) v_{n}-\left(I-v_{2} F_{2}\right) p\right\|^{2} \\
&=\left\|\left(v_{n}-p\right)-v_{2}\left(F_{2} v_{n}-F_{2} p\right)\right\|^{2} \\
& \leq\left\|v_{n}-p\right\|^{2}+v_{2}\left(v_{2}-2 \zeta_{2}\right)\left\|F_{2} v_{n}-F_{2} p\right\|^{2} \\
& \leq\left\|v_{n}-p\right\|^{2} .
\end{aligned}
$$

(This shows that $G: C \rightarrow C$ is a nonexpansive mapping.) Since $\left(\gamma_{n}+\delta_{n}\right) \xi \leq \gamma_{n}$ for all $n \geq 0$ and $T$ is $\xi$-strictly pseudocontractive, utilizing Lemma 2.4, we obtain from (3.1), (3.4), and (3.5)

$$
\begin{aligned}
\left\|y_{n}-p\right\| & =\left\|\beta_{n} x_{n}+\gamma_{n} G v_{n}+\delta_{n} T G v_{n}-p\right\| \\
& =\left\|\beta_{n}\left(x_{n}-p\right)+\gamma_{n}\left(G v_{n}-p\right)+\delta_{n}\left(T G v_{n}-p\right)\right\| \\
& \leq \beta_{n}\left\|x_{n}-p\right\|+\left\|\gamma_{n}\left(G v_{n}-p\right)+\delta_{n}\left(T G v_{n}-p\right)\right\| \\
& \leq \beta_{n}\left\|x_{n}-p\right\|+\left(\gamma_{n}+\delta_{n}\right)\left\|G v_{n}-p\right\| \\
& \leq \beta_{n}\left\|x_{n}-p\right\|+\left(\gamma_{n}+\delta_{n}\right)\left\|v_{n}-p\right\| \\
& \leq \beta_{n}\left\|x_{n}-p\right\|+\left(\gamma_{n}+\delta_{n}\right)\left\|x_{n}-p\right\| \\
& =\left\|x_{n}-p\right\| .
\end{aligned}
$$

Noticing the boundedness of $\left\{S x_{n}\right\}$, we get $\sup _{n \geq 0}\left\|\gamma S x_{n}-\mu F p\right\| \leq \widehat{M}$ for some $\widehat{M}>0$. Moreover, utilizing Lemma 2.7, we deduce from (3.1), (3.6), and $0<\gamma \leq \tau$ that, for all $n \geq 0$,

$$
\begin{aligned}
&\left\|x_{n+1}-p\right\| \\
&=\left\|\lambda_{n} \gamma\left(\alpha_{n} V x_{n}+\left(1-\alpha_{n}\right) S x_{n}\right)+\left(I-\lambda_{n} \mu F\right) y_{n}-p\right\| \\
&=\left\|\lambda_{n} \gamma\left(\alpha_{n} V x_{n}+\left(1-\alpha_{n}\right) S x_{n}\right)-\lambda_{n} \mu F p+\left(I-\lambda_{n} \mu F\right) y_{n}-\left(I-\lambda_{n} \mu F\right) p\right\| \\
& \leq\left\|\lambda_{n} \gamma\left(\alpha_{n} V x_{n}+\left(1-\alpha_{n}\right) S x_{n}\right)-\lambda_{n} \mu F p\right\|+\left\|\left(I-\lambda_{n} \mu F\right) y_{n}-\left(I-\lambda_{n} \mu F\right) p\right\| \\
&= \lambda_{n}\left\|\alpha_{n}\left(\gamma V x_{n}-\mu F p\right)+\left(1-\alpha_{n}\right)\left(\gamma S x_{n}-\mu F p\right)\right\| \\
& \quad+\left\|\left(I-\lambda_{n} \mu F\right) y_{n}-\left(I-\lambda_{n} \mu F\right) p\right\|
\end{aligned}
$$




$$
\begin{aligned}
\leq & \lambda_{n}\left[\alpha_{n}\left\|\gamma V x_{n}-\mu F p\right\|+\left(1-\alpha_{n}\right)\left\|\gamma S x_{n}-\mu F p\right\|\right]+\left(1-\lambda_{n} \tau\right)\left\|y_{n}-p\right\| \\
\leq & \lambda_{n}\left[\alpha_{n}\left(\gamma\left\|V x_{n}-V p\right\|+\|\gamma V p-\mu F p\|\right)+\left(1-\alpha_{n}\right)\left\|\gamma S x_{n}-\mu F p\right\|\right] \\
& +\left(1-\lambda_{n} \tau\right)\left\|x_{n}-p\right\| \\
\leq & \lambda_{n}\left[\alpha_{n} \gamma \rho\left\|x_{n}-p\right\|+\alpha_{n}\|\gamma V p-\mu F p\|+\left(1-\alpha_{n}\right) \widehat{M}\right]+\left(1-\lambda_{n} \tau\right)\left\|x_{n}-p\right\| \\
\leq & \lambda_{n}\left[\alpha_{n} \gamma \rho\left\|x_{n}-p\right\|+\max \{\widehat{M},\|\gamma V p-\mu F p\|\}\right]+\left(1-\lambda_{n} \tau\right)\left\|x_{n}-p\right\| \\
\leq & \lambda_{n} \gamma \rho\left\|x_{n}-p\right\|+\lambda_{n} \max \{\widehat{M},\|\gamma V p-\mu F p\|\}+\left(1-\lambda_{n} \tau\right)\left\|x_{n}-p\right\| \\
= & \left(1-\lambda_{n}(\tau-\gamma \rho)\right)\left\|x_{n}-p\right\|+\lambda_{n} \max \{\widehat{M},\|\gamma V p-\mu F p\|\} \\
= & \left(1-\lambda_{n}(\tau-\gamma \rho)\right)\left\|x_{n}-p\right\|+\lambda_{n}(\tau-\gamma \rho) \max \left\{\frac{\widehat{M}}{\tau-\gamma \rho}, \frac{\|\gamma V p-\mu F p\|}{\tau-\gamma \rho}\right\} \\
\leq & \max \left\{\left\|x_{n}-p\right\|, \frac{\widehat{M}}{\tau-\gamma \rho}, \frac{\|\gamma V p-\mu F p\|}{\tau-\gamma \rho}\right\} .
\end{aligned}
$$

By induction, we obtain

$$
\left\|x_{n}-p\right\| \leq \max \left\{\left\|x_{0}-p\right\|, \frac{\widehat{M}}{\tau-\gamma \rho}, \frac{\|\gamma V p-\mu F p\|}{\tau-\gamma \rho}\right\}, \quad \forall n \geq 0
$$

Thus, $\left\{x_{n}\right\}$ is bounded and so are the sequences $\left\{u_{n}\right\},\left\{v_{n}\right\}$ and $\left\{y_{n}\right\}$.

Step 2. We prove that $\lim _{n \rightarrow \infty} \frac{\left\|x_{n+1}-x_{n}\right\|}{\alpha_{n}}=0$.

Indeed, utilizing (2.1) and (2.3), we obtain

$$
\begin{aligned}
\| v_{n+1}- & v_{n} \| \\
= & \left\|\Lambda_{n+1}^{N} u_{n+1}-\Lambda_{n}^{N} u_{n}\right\| \\
= & \left\|J_{R_{N}, \lambda_{N, n+1}}\left(I-\lambda_{N, n+1} B_{N}\right) \Lambda_{n+1}^{N-1} u_{n+1}-J_{R_{N}, \lambda_{N, n}}\left(I-\lambda_{N, n} B_{N}\right) \Lambda_{n}^{N-1} u_{n}\right\| \\
\leq & \left\|J_{R_{N}, \lambda_{N, n+1}}\left(I-\lambda_{N, n+1} B_{N}\right) \Lambda_{n+1}^{N-1} u_{n+1}-J_{R_{N}, \lambda_{N, n+1}}\left(I-\lambda_{N, n} B_{N}\right) \Lambda_{n+1}^{N-1} u_{n+1}\right\| \\
& +\left\|J_{R_{N}, \lambda_{N, n+1}}\left(I-\lambda_{N, n} B_{N}\right) \Lambda_{n+1}^{N-1} u_{n+1}-J_{R_{N}, \lambda_{N, n}}\left(I-\lambda_{N, n} B_{N}\right) \Lambda_{n}^{N-1} u_{n}\right\| \\
\leq & \left\|\left(I-\lambda_{N, n+1} B_{N}\right) \Lambda_{n+1}^{N-1} u_{n+1}-\left(I-\lambda_{N, n} B_{N}\right) \Lambda_{n+1}^{N-1} u_{n+1}\right\| \\
& +\left\|\left(I-\lambda_{N, n} B_{N}\right) \Lambda_{n+1}^{N-1} u_{n+1}-\left(I-\lambda_{N, n} B_{N}\right) \Lambda_{n}^{N-1} u_{n}\right\|+\left|\lambda_{N, n+1}-\lambda_{N, n}\right| \\
& \times\left(\frac{1}{\lambda_{N, n+1}}\left\|J_{R_{N}, \lambda_{N, n+1}}\left(I-\lambda_{N, n} B_{N}\right) \Lambda_{n+1}^{N-1} u_{n+1}-\left(I-\lambda_{N, n} B_{N}\right) \Lambda_{n}^{N-1} u_{n}\right\|\right. \\
& \left.+\frac{1}{\lambda_{N, n}}\left\|\left(I-\lambda_{N, n} B_{N}\right) \Lambda_{n+1}^{N-1} u_{n+1}-J_{R_{N}, \lambda_{N, n}}\left(I-\lambda_{N, n} B_{N}\right) \Lambda_{n}^{N-1} u_{n}\right\|\right) \\
\leq & \left|\lambda_{N, n+1}-\lambda_{N, n}\right|\left(\left\|B_{N} \Lambda_{n+1}^{N-1} u_{n+1}\right\|+\widetilde{M}\right)+\left\|\Lambda_{n+1}^{N-1} u_{n+1}-\Lambda_{n}^{N-1} u_{n}\right\| \\
\leq & \left|\lambda_{N, n+1}-\lambda_{N, n}\right|\left(\left\|B_{N} \Lambda_{n+1}^{N-1} u_{n+1}\right\|+\widetilde{M}\right) \\
& +\left|\lambda_{N-1, n+1}-\lambda_{N-1, n}\right|\left(\left\|B_{N-1} \Lambda_{n+1}^{N-2} u_{n+1}\right\|+\widetilde{M}\right)+\left\|\Lambda_{n+1}^{N-2} u_{n+1}-\Lambda_{n}^{N-2} u_{n}\right\| \\
& \quad \ldots \\
\leq & \left|\lambda_{N, n+1}-\lambda_{N, n}\right|\left(\left\|B_{N} \Lambda_{n+1}^{N-1} u_{n+1}\right\|+\widetilde{M}\right) \\
& +\left|\lambda_{N-1, n+1}-\lambda_{N-1, n}\right|\left(\left\|B_{N-1} \Lambda_{n+1}^{N-2} u_{n+1}\right\|+\widetilde{M}\right)
\end{aligned}
$$




$$
\begin{aligned}
& +\cdots+\left|\lambda_{1, n+1}-\lambda_{1, n}\right|\left(\left\|B_{1} \Lambda_{n+1}^{0} u_{n+1}\right\|+\tilde{M}\right)+\left\|\Lambda_{n+1}^{0} u_{n+1}-\Lambda_{n}^{0} u_{n}\right\| \\
\leq & \widetilde{M}_{0} \sum_{i=1}^{N}\left|\lambda_{i, n+1}-\lambda_{i, n}\right|+\left\|u_{n+1}-u_{n}\right\|
\end{aligned}
$$

where

$$
\begin{aligned}
& \sup _{n \geq 0}\left\{\frac{1}{\lambda_{N, n+1}}\left\|J_{R_{N}, \lambda_{N, n+1}}\left(I-\lambda_{N, n} B_{N}\right) \Lambda_{n+1}^{N-1} u_{n+1}-\left(I-\lambda_{N, n} B_{N}\right) \Lambda_{n}^{N-1} u_{n}\right\|\right. \\
& \left.\quad+\frac{1}{\lambda_{N, n}}\left\|\left(I-\lambda_{N, n} B_{N}\right) \Lambda_{n+1}^{N-1} u_{n+1}-J_{R_{N}, \lambda_{N, n}}\left(I-\lambda_{N, n} B_{N}\right) \Lambda_{n}^{N-1} u_{n}\right\|\right\} \leq \tilde{M}
\end{aligned}
$$

for some $\tilde{M}>0$ and $\sup _{n \geq 0}\left\{\sum_{i=1}^{N}\left\|B_{i} \Lambda_{n+1}^{i-1} u_{n+1}\right\|+\widetilde{M}\right\} \leq \widetilde{M}_{0}$ for some $\widetilde{M}_{0}>0$. Utilizing Proposition 2.2(ii), (v), we have

$$
\begin{aligned}
& \left\|u_{n+1}-u_{n}\right\| \\
& =\left\|\Delta_{n+1}^{M} x_{n+1}-\Delta_{n}^{M} x_{n}\right\|
\end{aligned}
$$

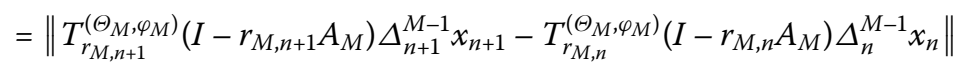

$$
\begin{aligned}
& \leq\left\|T_{r_{M, n+1}}^{\left(\Theta_{M}, \varphi_{M}\right)}\left(I-r_{M, n+1} A_{M}\right) \Delta_{n+1}^{M-1} x_{n+1}-T_{r_{M, n}}^{\left(\Theta_{M}, \varphi_{M}\right)}\left(I-r_{M, n} A_{M}\right) \Delta_{n+1}^{M-1} x_{n+1}\right\|
\end{aligned}
$$

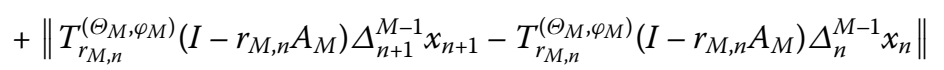

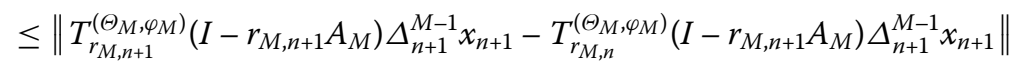

$$
\begin{aligned}
& +\left\|T_{r_{M, n}}^{\left(\Theta_{M}, \varphi_{M}\right)}\left(I-r_{M, n+1} A_{M}\right) \Delta_{n+1}^{M-1} x_{n+1}-T_{r_{M, n}}^{\left(\Theta_{M}, \varphi_{M}\right)}\left(I-r_{M, n} A_{M}\right) \Delta_{n+1}^{M-1} x_{n+1}\right\| \\
& +\left\|\left(I-r_{M, n} A_{M}\right) \Delta_{n+1}^{M-1} x_{n+1}-\left(I-r_{M, n} A_{M}\right) \Delta_{n}^{M-1} x_{n}\right\|
\end{aligned}
$$

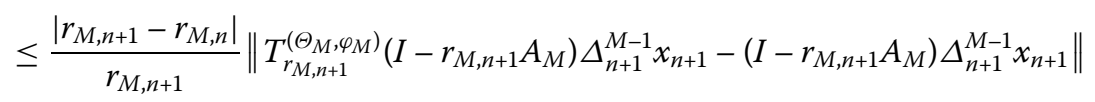

$$
\begin{aligned}
& +\left|r_{M, n+1}-r_{M, n}\right|\left\|A_{M} \Delta_{n+1}^{M-1} x_{n+1}\right\|+\left\|\Delta_{n+1}^{M-1} x_{n+1}-\Delta_{n}^{M-1} x_{n}\right\| \\
& =\left|r_{M, n+1}-r_{M, n}\right|\left[\left\|A_{M} \Delta_{n+1}^{M-1} x_{n+1}\right\|+\frac{1}{r_{M, n+1}} \| T_{r_{M, n+1}^{\left(\Theta_{M}, \varphi_{M}\right)}\left(I-r_{M, n+1} A_{M}\right) \Delta_{n+1}^{M-1} x_{n+1}}\right. \\
& \left.-\left(I-r_{M, n+1} A_{M}\right) \Delta_{n+1}^{M-1} x_{n+1} \|\right]+\left\|\Delta_{n+1}^{M-1} x_{n+1}-\Delta_{n}^{M-1} x_{n}\right\| \\
& \leq\left|r_{M, n+1}-r_{M, n}\right|\left[\left\|A_{M} \Delta_{n+1}^{M-1} x_{n+1}\right\|+\frac{1}{r_{M, n+1}} \| T_{r_{M, n+1}^{\left(\Theta_{M}, \varphi_{M}\right)}\left(I-r_{M, n+1} A_{M}\right) \Delta_{n+1}^{M-1} x_{n+1}}\right. \\
& \left.-\left(I-r_{M, n+1} A_{M}\right) \Delta_{n+1}^{M-1} x_{n+1} \|\right]+\cdots+\left|r_{1, n+1}-r_{1, n}\right|\left[\left\|A_{1} \Delta_{n+1}^{0} x_{n+1}\right\|\right.
\end{aligned}
$$

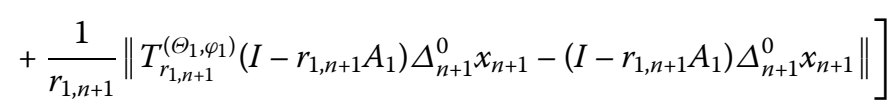

$$
\begin{aligned}
& +\left\|\Delta_{n+1}^{0} x_{n+1}-\Delta_{n}^{0} x_{n}\right\| \\
& \leq \tilde{M}_{1} \sum_{k=1}^{M}\left|r_{k, n+1}-r_{k, n}\right|+\left\|x_{n+1}-x_{n}\right\|
\end{aligned}
$$


where $\tilde{M}_{1}>0$ is a constant such that, for each $n \geq 0$,

$$
\begin{aligned}
& \sum_{k=1}^{M}\left[\left\|A_{k} \Delta_{n+1}^{k-1} x_{n+1}\right\|+\frac{1}{r_{k, n+1}}\left\|T_{r_{k, n+1}}^{\left(\Theta_{k}, \varphi_{k}\right)}\left(I-r_{k, n+1} A_{k}\right) \Delta_{n+1}^{k-1} x_{n+1}-\left(I-r_{k, n+1} A_{k}\right) \Delta_{n+1}^{k-1} x_{n+1}\right\|\right] \\
& \quad \leq \widetilde{M}_{1} .
\end{aligned}
$$

Furthermore, define $y_{n}=\beta_{n} x_{n}+\left(1-\beta_{n}\right) w_{n}$ for all $n \geq 0$. It follows that

$$
\begin{aligned}
& w_{n+1}-w_{n} \\
&= \frac{y_{n+1}-\beta_{n+1} x_{n+1}}{1-\beta_{n+1}}-\frac{y_{n}-\beta_{n} x_{n}}{1-\beta_{n}} \\
&= \frac{\gamma_{n+1} G v_{n+1}+\delta_{n+1} T G v_{n+1}}{1-\beta_{n+1}}-\frac{\gamma_{n} G v_{n}+\delta_{n} T G v_{n}}{1-\beta_{n}} \\
&= \frac{\gamma_{n+1}\left(G v_{n+1}-G v_{n}\right)+\delta_{n+1}\left(T G v_{n+1}-T G v_{n}\right)}{1-\beta_{n+1}} \\
&+\left(\frac{\gamma_{n+1}}{1-\beta_{n+1}}-\frac{\gamma_{n}}{1-\beta_{n}}\right) G v_{n}+\left(\frac{\delta_{n+1}}{1-\beta_{n+1}}-\frac{\delta_{n}}{1-\beta_{n}}\right) T G v_{n} .
\end{aligned}
$$

Since $\left(\gamma_{n}+\delta_{n}\right) \xi \leq \gamma_{n}$ for all $n \geq 0$, utilizing Lemma 2.4, we have

$$
\left\|\gamma_{n+1}\left(G v_{n+1}-G v_{n}\right)+\delta_{n+1}\left(T G v_{n+1}-T G v_{n}\right)\right\| \leq\left(\gamma_{n+1}+\delta_{n+1}\right)\left\|G v_{n+1}-G v_{n}\right\| .
$$

Hence, it follows from (3.7)-(3.10) that

$$
\begin{aligned}
\left\|w_{n+1}-w_{n}\right\| & \\
\leq & \frac{\left\|\gamma_{n+1}\left(G v_{n+1}-G v_{n}\right)+\delta_{n+1}\left(T G v_{n+1}-T G v_{n}\right)\right\|}{1-\beta_{n+1}} \\
& +\left|\frac{\gamma_{n+1}}{1-\beta_{n+1}}-\frac{\gamma_{n}}{1-\beta_{n}}\right|\left\|G v_{n}\right\|+\left|\frac{\delta_{n+1}}{1-\beta_{n+1}}-\frac{\delta_{n}}{1-\beta_{n}}\right|\left\|T G v_{n}\right\| \\
\leq & \frac{\left(\gamma_{n+1}+\delta_{n+1}\right)}{1-\beta_{n+1}}\left\|G v_{n+1}-G v_{n}\right\|+\left|\frac{\gamma_{n+1}}{1-\beta_{n+1}}-\frac{\gamma_{n}}{1-\beta_{n}}\right|\left(\left\|G v_{n}\right\|+\left\|T G v_{n}\right\|\right) \\
= & \left\|G v_{n+1}-G v_{n}\right\|+\left|\frac{\gamma_{n+1}}{1-\beta_{n+1}}-\frac{\gamma_{n}}{1-\beta_{n}}\right|\left(\left\|G v_{n}\right\|+\left\|T G v_{n}\right\|\right) \\
\leq & \left\|v_{n+1}-v_{n}\right\|+\left|\frac{\gamma_{n+1}}{1-\beta_{n+1}}-\frac{\gamma_{n}}{1-\beta_{n}}\right|\left(\left\|G v_{n}\right\|+\left\|T G v_{n}\right\|\right) \\
\leq & \widetilde{M}_{0} \sum_{i=1}^{N}\left|\lambda_{i, n+1}-\lambda_{i, n}\right|+\left\|u_{n+1}-u_{n}\right\|+\left|\frac{\gamma_{n+1}}{1-\beta_{n+1}}-\frac{\gamma_{n}}{1-\beta_{n}}\right|\left(\left\|G v_{n}\right\|+\left\|T G v_{n}\right\|\right) \\
\leq & \widetilde{M}_{0} \sum_{i=1}^{N}\left|\lambda_{i, n+1}-\lambda_{i, n}\right|+\widetilde{M}_{1} \sum_{k=1}^{M}\left|r_{k, n+1}-r_{k, n}\right|+\left\|x_{n+1}-x_{n}\right\| \\
& +\left|\frac{\gamma_{n+1}}{1-\beta_{n+1}}-\frac{\gamma_{n}}{1-\beta_{n}}\right|\left(\left\|G v_{n}\right\|+\left\|T G v_{n}\right\|\right) .
\end{aligned}
$$

In the meantime, simple calculation shows that

$$
y_{n+1}-y_{n}=\beta_{n}\left(x_{n+1}-x_{n}\right)+\left(1-\beta_{n}\right)\left(w_{n+1}-w_{n}\right)+\left(\beta_{n+1}-\beta_{n}\right)\left(x_{n+1}-w_{n+1}\right) .
$$


So, it follows from (3.11) that

$$
\begin{aligned}
\left\|y_{n+1}-y_{n}\right\| & \\
\leq & \beta_{n}\left\|x_{n+1}-x_{n}\right\|+\left(1-\beta_{n}\right)\left\|w_{n+1}-w_{n}\right\|+\left|\beta_{n+1}-\beta_{n}\right|\left\|x_{n+1}-w_{n+1}\right\| \\
\leq & \beta_{n}\left\|x_{n+1}-x_{n}\right\|+\left(1-\beta_{n}\right)\left[\tilde{M}_{0} \sum_{i=1}^{N}\left|\lambda_{i, n+1}-\lambda_{i, n}\right|+\tilde{M}_{1} \sum_{k=1}^{M}\left|r_{k, n+1}-r_{k, n}\right|+\left\|x_{n+1}-x_{n}\right\|\right. \\
& \left.+\left|\frac{\gamma_{n+1}}{1-\beta_{n+1}}-\frac{\gamma_{n}}{1-\beta_{n}}\right|\left(\left\|G v_{n}\right\|+\left\|T G v_{n}\right\|\right)\right]+\left|\beta_{n+1}-\beta_{n}\right||| x_{n+1}-w_{n+1} \| \\
\leq & \left\|x_{n+1}-x_{n}\right\|+\tilde{M}_{0} \sum_{i=1}^{N}\left|\lambda_{i, n+1}-\lambda_{i, n}\right|+\tilde{M}_{1} \sum_{k=1}^{M}\left|r_{k, n+1}-r_{k, n}\right| \\
& +\frac{\left|\gamma_{n+1}-\gamma_{n}\right|\left(1-\beta_{n}\right)+\gamma_{n}\left|\beta_{n+1}-\beta_{n}\right|}{1-\beta_{n+1}}\left(\left\|G v_{n}\right\|+\left\|T G v_{n}\right\|\right) \\
& +\left|\beta_{n+1}-\beta_{n}\right|\left\|x_{n+1}-w_{n+1}\right\| \\
\leq & \left\|x_{n+1}-x_{n}\right\|+\tilde{M}_{0} \sum_{i=1}^{N}\left|\lambda_{i, n+1}-\lambda_{i, n}\right|+\tilde{M}_{1} \sum_{k=1}^{M}\left|r_{k, n+1}-r_{k, n}\right| \\
& +\left|\gamma_{n+1}-\gamma_{n}\right| \frac{\left\|G v_{n}\right\|+\left\|T G v_{n}\right\|}{1-b}+\left|\beta_{n+1}-\beta_{n}\right|\left(\left\|x_{n+1}-w_{n+1}\right\|+\frac{\left\|G v_{n}\right\|+\left\|T G v_{n}\right\|}{1-b}\right) \\
\leq & \left\|x_{n+1}-x_{n}\right\| \\
& +\widetilde{M}_{2}\left(\sum_{i=1}^{N}\left|\lambda_{i, n+1}-\lambda_{i, n}\right|+\sum_{k=1}^{M}\left|r_{k, n+1}-r_{k, n}\right|+\left|\gamma_{n+1}-\gamma_{n}\right|+\left|\beta_{n+1}-\beta_{n}\right|\right),
\end{aligned}
$$

where $\sup _{n \geq 0}\left\{\left\|x_{n+1}-w_{n+1}\right\|+\frac{\left\|G v_{n}\right\|+\left\|T G v_{n}\right\|}{1-b}+\widetilde{M}_{0}+\widetilde{M}_{1}\right\} \leq \widetilde{M}_{2}$ for some $\widetilde{M}_{2}>0$.

On the other hand, define $z_{n}:=\alpha_{n} V x_{n}+\left(1-\alpha_{n}\right) S x_{n}$ for all $n \geq 0$. Then as well known $x_{n+1}=\lambda_{n} \gamma z_{n}+\left(I-\lambda_{n} \mu F\right) y_{n}$ for all $n \geq 0$. Simple calculations show that

$$
\left\{\begin{aligned}
z_{n+1}-z_{n}= & \left(\alpha_{n+1}-\alpha_{n}\right)\left(V x_{n}-S x_{n}\right)+\alpha_{n+1}\left(V x_{n+1}-V x_{n}\right) \\
+ & \left(1-\alpha_{n+1}\right)\left(S x_{n+1}-S x_{n}\right) \\
x_{n+2}-x_{n+1}= & \left(\lambda_{n+1}-\lambda_{n}\right)\left(\gamma z_{n}-\mu F y_{n}\right)+\lambda_{n+1} \gamma\left(z_{n+1}-z_{n}\right) \\
& +\left(I-\lambda_{n+1} \mu F\right) y_{n+1}-\left(I-\lambda_{n+1} \mu F\right) y_{n} .
\end{aligned}\right.
$$

Since $V$ is a $\rho$-contraction with coefficient $\rho \in[0,1)$ and $S$ is a nonexpansive mapping, we conclude that

$$
\begin{aligned}
\left\|z_{n+1}-z_{n}\right\| & \leq\left|\alpha_{n+1}-\alpha_{n}\right|\left\|V x_{n}-S x_{n}\right\|+\alpha_{n+1}\left\|V x_{n+1}-V x_{n}\right\|+\left(1-\alpha_{n+1}\right)\left\|S x_{n+1}-S x_{n}\right\| \\
& \leq\left|\alpha_{n+1}-\alpha_{n}\right|\left\|V x_{n}-S x_{n}\right\|+\alpha_{n+1} \rho\left\|x_{n+1}-x_{n}\right\|+\left(1-\alpha_{n+1}\right)\left\|x_{n+1}-x_{n}\right\| \\
& =\left(1-\alpha_{n+1}(1-\rho)\right)\left\|x_{n+1}-x_{n}\right\|+\left|\alpha_{n+1}-\alpha_{n}\right|\left\|V x_{n}-S x_{n}\right\|,
\end{aligned}
$$

which, together with (3.12) and $0<\gamma \leq \tau$, implies that

$$
\begin{aligned}
& \left\|x_{n+2}-x_{n+1}\right\| \\
& \quad \leq\left|\lambda_{n+1}-\lambda_{n}\right|\left\|\gamma z_{n}-\mu F y_{n}\right\|+\lambda_{n+1} \gamma\left\|z_{n+1}-z_{n}\right\|
\end{aligned}
$$




$$
\begin{aligned}
& +\left\|\left(I-\lambda_{n+1} \mu F\right) y_{n+1}-\left(I-\lambda_{n+1} \mu F\right) y_{n}\right\| \\
\leq & \left|\lambda_{n+1}-\lambda_{n}\right|\left\|\gamma z_{n}-\mu F y_{n}\right\|+\lambda_{n+1} \gamma\left\|z_{n+1}-z_{n}\right\|+\left(1-\lambda_{n+1} \tau\right)\left\|y_{n+1}-y_{n}\right\| \\
\leq & \left|\lambda_{n+1}-\lambda_{n}\right|\left\|\gamma z_{n}-\mu F y_{n}\right\|+\lambda_{n+1} \gamma\left[\left(1-\alpha_{n+1}(1-\rho)\right)\left\|x_{n+1}-x_{n}\right\|\right. \\
& \left.+\left|\alpha_{n+1}-\alpha_{n}\right|\left\|V x_{n}-S x_{n}\right\|\right]+\left(1-\lambda_{n+1} \tau\right)\left[\left\|x_{n+1}-x_{n}\right\|+\tilde{M}_{2}\left(\sum_{i=1}^{N}\left|\lambda_{i, n+1}-\lambda_{i, n}\right|\right.\right. \\
& \left.\left.+\sum_{k=1}^{M}\left|r_{k, n+1}-r_{k, n}\right|+\left|\gamma_{n+1}-\gamma_{n}\right|+\left|\beta_{n+1}-\beta_{n}\right|\right)\right] \\
\leq & \left(1-\lambda_{n+1}(\tau-\gamma)-\lambda_{n+1} \alpha_{n+1} \gamma(1-\rho)\right)\left\|x_{n+1}-x_{n}\right\|+\left|\lambda_{n+1}-\lambda_{n}\right|\left\|\gamma z_{n}-\mu F y_{n}\right\| \\
& +\left|\alpha_{n+1}-\alpha_{n}\right|\left\|V x_{n}-S x_{n}\right\|+\tilde{M}_{2}\left(\sum_{i=1}^{N}\left|\lambda_{i, n+1}-\lambda_{i, n}\right|\right. \\
& \left.+\sum_{k=1}^{M}\left|r_{k, n+1}-r_{k, n}\right|+\left|\gamma_{n+1}-\gamma_{n}\right|+\left|\beta_{n+1}-\beta_{n}\right|\right) \\
\leq & \left(1-\lambda_{n+1} \alpha_{n+1} \gamma(1-\rho)\right)\left\|x_{n+1}-x_{n}\right\|+\tilde{M}_{3}\left\{\sum_{i=1}^{N}\left|\lambda_{i, n+1}-\lambda_{i, n}\right|+\sum_{k=1}^{M}\left|r_{k, n+1}-r_{k, n}\right|\right. \\
& \left.+\left|\lambda_{n+1}-\lambda_{n}\right|+\left|\alpha_{n+1}-\alpha_{n}\right|+\left|\beta_{n+1}-\beta_{n}\right|+\left|\gamma_{n+1}-\gamma_{n}\right|\right\},
\end{aligned}
$$

where $\sup _{n \geq 0}\left\{\left\|\gamma z_{n}-\mu F y_{n}\right\|+\left\|V x_{n}-S x_{n}\right\|+\widetilde{M}_{2}\right\} \leq \widetilde{M}_{3}$ for some $\widetilde{M}_{3}>0$. Consequently,

$$
\begin{aligned}
\frac{\left\|x_{n+1}-x_{n}\right\|}{\alpha_{n}} & \left(1-\lambda_{n} \alpha_{n} \gamma(1-\rho)\right) \frac{|| x_{n}-x_{n-1} \|}{\alpha_{n}}+\tilde{M}_{3}\left\{\sum_{i=1}^{N} \frac{\left|\lambda_{i, n}-\lambda_{i, n-1}\right|}{\alpha_{n}}+\sum_{k=1}^{M} \frac{\left|r_{k, n}-r_{k, n-1}\right|}{\alpha_{n}}\right. \\
& \left.+\frac{\left|\lambda_{n}-\lambda_{n-1}\right|}{\alpha_{n}}+\frac{\left|\alpha_{n}-\alpha_{n-1}\right|}{\alpha_{n}}+\frac{\left|\beta_{n}-\beta_{n-1}\right|}{\alpha_{n}}+\frac{\left|\gamma_{n}-\gamma_{n-1}\right|}{\alpha_{n}}\right\} \\
= & \left(1-\lambda_{n} \alpha_{n} \gamma(1-\rho)\right) \frac{|| x_{n}-x_{n-1} \mid}{\alpha_{n-1}}+\left(1-\lambda_{n} \alpha_{n} \gamma(1-\rho)\right) \| x_{n}-x_{n-1}||\left(\frac{1}{\alpha_{n}}-\frac{1}{\alpha_{n-1}}\right) \\
& +\tilde{M}_{3}\left\{\sum_{i=1}^{N} \frac{\left|\lambda_{i, n}-\lambda_{i, n-1}\right|}{\alpha_{n}}+\sum_{k=1}^{M} \frac{\left|r_{k, n}-r_{k, n-1}\right|}{\alpha_{n}}+\frac{\left|\lambda_{n}-\lambda_{n-1}\right|}{\alpha_{n}}\right. \\
& \left.+\frac{\left|\alpha_{n}-\alpha_{n-1}\right|}{\alpha_{n}}+\frac{\left|\beta_{n}-\beta_{n-1}\right|}{\alpha_{n}}+\frac{\left|\gamma_{n}-\gamma_{n-1}\right|}{\alpha_{n}}\right\} \\
\leq & \left(1-\lambda_{n} \alpha_{n} \gamma(1-\rho)\right) \frac{|| x_{n}-x_{n-1} \|}{\alpha_{n-1}}+\lambda_{n} \alpha_{n} \gamma(1-\rho) \cdot \frac{\tilde{M}_{4}}{\gamma(1-\rho)}\left\{\frac{1}{\lambda_{n} \alpha_{n}}\left|\frac{1}{\alpha_{n}}-\frac{1}{\alpha_{n-1}}\right|\right. \\
& +\sum_{i=1}^{N} \frac{\left|\lambda_{i, n}-\lambda_{i, n-1}\right|}{\lambda_{n} \alpha_{n}^{2}}+\sum_{k=1}^{M} \frac{\left|r_{k, n}-r_{k, n-1}\right|}{\lambda_{n} \alpha_{n}^{2}}+\frac{1}{\alpha_{n}^{2}} \mid 1-\frac{\lambda_{n-1} \mid}{\lambda_{n} \mid} \\
& \left.+\frac{1}{\lambda_{n} \alpha_{n}}\left|1-\frac{\alpha_{n-1}}{\alpha_{n}}\right|+\frac{\left|\beta_{n}-\beta_{n-1}\right|}{\lambda_{n} \alpha_{n}^{2}}+\frac{\left|\gamma_{n}-\gamma_{n-1}\right|}{\lambda_{n} \alpha_{n}^{2}}\right\},
\end{aligned}
$$


where $\sup _{n \geq 1}\left\{\left\|x_{n}-x_{n-1}\right\|+\widetilde{M}_{3}\right\} \leq \widetilde{M}_{4}$ for some $\widetilde{M}_{4}>0$. From conditions (i)-(iv), it follows that $\sum_{n=0}^{\infty} \lambda_{n} \alpha_{n} \gamma(1-\rho)=\infty$ and

$$
\begin{gathered}
\lim _{n \rightarrow \infty} \frac{\widetilde{M}_{4}}{\gamma(1-\rho)}\left\{\frac{1}{\lambda_{n} \alpha_{n}}\left|\frac{1}{\alpha_{n}}-\frac{1}{\alpha_{n-1}}\right|+\sum_{i=1}^{N} \frac{\left|\lambda_{i, n}-\lambda_{i, n-1}\right|}{\lambda_{n} \alpha_{n}^{2}}+\sum_{k=1}^{M} \frac{\left|r_{k, n}-r_{k, n-1}\right|}{\lambda_{n} \alpha_{n}^{2}}\right. \\
\left.+\frac{1}{\alpha_{n}^{2}}\left|1-\frac{\lambda_{n-1}}{\lambda_{n}}\right|+\frac{1}{\lambda_{n} \alpha_{n}}\left|1-\frac{\alpha_{n-1}}{\alpha_{n}}\right|+\frac{\left|\beta_{n}-\beta_{n-1}\right|}{\lambda_{n} \alpha_{n}^{2}}+\frac{\left|\gamma_{n}-\gamma_{n-1}\right|}{\lambda_{n} \alpha_{n}^{2}}\right\}=0 .
\end{gathered}
$$

Thus, utilizing Lemma 2.8, we immediately conclude that

$$
\lim _{n \rightarrow \infty} \frac{\left\|x_{n+1}-x_{n}\right\|}{\alpha_{n}}=0
$$

So, from $\alpha_{n} \rightarrow 0$, it follows that

$$
\lim _{n \rightarrow \infty}\left\|x_{n+1}-x_{n}\right\|=0
$$

Step 3. We prove that $\lim _{n \rightarrow \infty}\left\|x_{n}-u_{n}\right\|=0, \lim _{n \rightarrow \infty}\left\|x_{n}-v_{n}\right\|=0, \lim _{n \rightarrow \infty}\left\|v_{n}-G v_{n}\right\|=$ 0 , and $\lim _{n \rightarrow \infty}\left\|v_{n}-T v_{n}\right\|=0$.

Indeed, utilizing Lemmas 2.1 and 2.2(b), from (3.1), (3.4)-(3.5), and $0<\gamma \leq \tau$, we deduce that

$$
\begin{aligned}
\left\|y_{n}-p\right\|^{2} & \left\|\beta_{n} x_{n}+\gamma_{n} G v_{n}+\delta_{n} T G v_{n}-p\right\|^{2} \\
= & \left\|\beta_{n}\left(x_{n}-p\right)+\left(1-\beta_{n}\right)\left(\frac{\gamma_{n} G v_{n}+\delta_{n} T G v_{n}}{1-\beta_{n}}-p\right)\right\|^{2} \\
= & \beta_{n}\left\|x_{n}-p\right\|^{2}+\left(1-\beta_{n}\right)\left\|\frac{\gamma_{n} G v_{n}+\delta_{n} T G v_{n}}{1-\beta_{n}}-p\right\|^{2} \\
& -\beta_{n}\left(1-\beta_{n}\right)\left\|\frac{\gamma_{n} G v_{n}+\delta_{n} T G v_{n}}{1-\beta_{n}}-x_{n}\right\|^{2} \\
= & \beta_{n}\left\|x_{n}-p\right\|^{2}+\left(1-\beta_{n}\right)\left\|\frac{\gamma_{n}\left(G v_{n}-p\right)+\delta_{n}\left(T G v_{n}-p\right)}{1-\beta_{n}}\right\|^{2} \\
& -\beta_{n}\left(1-\beta_{n}\right)\left\|\frac{y_{n}-x_{n}}{1-\beta_{n}}\right\| \|^{2} \\
\leq & \beta_{n}\left\|x_{n}-p\right\|^{2}+\left(1-\beta_{n}\right) \frac{\left(\gamma_{n}+\delta_{n}\right)^{2}\left\|G v_{n}-p\right\|^{2}}{\left(1-\beta_{n}\right)^{2}}-\frac{\beta_{n}}{1-\beta_{n}}\left\|y_{n}-x_{n}\right\|^{2} \\
= & \beta_{n}\left\|x_{n}-p\right\|^{2}+\left(1-\beta_{n}\right)\left\|G v_{n}-p\right\|^{2}-\frac{\beta_{n}}{1-\beta_{n}}\left\|y_{n}-x_{n}\right\|^{2} \\
\leq & \beta_{n}\left\|x_{n}-p\right\|^{2}+\left(1-\beta_{n}\right)\left\|v_{n}-p\right\|^{2}-\frac{\beta_{n}}{1-\beta_{n}}\left\|y_{n}-x_{n}\right\|^{2} \\
\leq & \beta_{n}\left\|x_{n}-p\right\|^{2}+\left(1-\beta_{n}\right)\left\|x_{n}-p\right\|^{2}-\frac{\beta_{n}}{1-\beta_{n}}\left\|y_{n}-x_{n}\right\|^{2} \\
= & \left\|x_{n}-p\right\|^{2}-\frac{\beta_{n}}{1-\beta_{n}}\left\|y_{n}-x_{n}\right\|^{2},
\end{aligned}
$$


and hence

$$
\begin{aligned}
& \left\|x_{n+1}-p\right\|^{2} \\
& =\left\|\lambda_{n} \gamma\left(\alpha_{n} V x_{n}+\left(1-\alpha_{n}\right) S x_{n}\right)+\left(I-\lambda_{n} \mu F\right) y_{n}-p\right\|^{2} \\
& =\left\|\lambda_{n} \gamma\left(\alpha_{n} V x_{n}+\left(1-\alpha_{n}\right) S x_{n}\right)-\lambda_{n} \mu F p+\left(I-\lambda_{n} \mu F\right) y_{n}-\left(I-\lambda_{n} \mu F\right) p\right\|^{2} \\
& =\left\|\lambda_{n}\left[\alpha_{n}\left(\gamma V x_{n}-\mu F p\right)+\left(1-\alpha_{n}\right)\left(\gamma S x_{n}-\mu F p\right)\right]+\left(I-\lambda_{n} \mu F\right) y_{n}-\left(I-\lambda_{n} \mu F\right) p\right\|^{2} \\
& =\| \lambda_{n}\left[\alpha_{n}\left(\gamma V x_{n}-\gamma V p\right)+\left(1-\alpha_{n}\right)\left(\gamma S x_{n}-\gamma S p\right)\right]+\left(I-\lambda_{n} \mu F\right) y_{n}-\left(I-\lambda_{n} \mu F\right) p \\
& +\lambda_{n}\left[\alpha_{n}(\gamma V p-\mu F p)+\left(1-\alpha_{n}\right)(\gamma S p-\mu F p)\right] \|^{2} \\
& \leq \| \lambda_{n}\left[\alpha_{n}\left(\gamma V x_{n}-\gamma V p\right)+\left(1-\alpha_{n}\right)\left(\gamma S x_{n}-\gamma S p\right)\right] \\
& +\left(I-\lambda_{n} \mu F\right) y_{n}-\left(I-\lambda_{n} \mu F\right) p \|^{2} \\
& +2 \lambda_{n} \alpha_{n}\left\langle(\gamma V p-\mu F p), x_{n+1}-p\right\rangle+2 \lambda_{n}\left(1-\alpha_{n}\right)\left\langle(\gamma S p-\mu F p), x_{n+1}-p\right\rangle \\
& \leq\left[\lambda_{n}\left\|\alpha_{n}\left(\gamma V x_{n}-\gamma V p\right)+\left(1-\alpha_{n}\right)\left(\gamma S x_{n}-\gamma S p\right)\right\|\right. \\
& \left.+\left\|\left(I-\lambda_{n} \mu F\right) y_{n}-\left(I-\lambda_{n} \mu F\right) p\right\|\right]^{2} \\
& +2 \lambda_{n} \alpha_{n}\left\langle(\gamma V p-\mu F p), x_{n+1}-p\right\rangle+2 \lambda_{n}\left(1-\alpha_{n}\right)\left\langle(\gamma S p-\mu F p), x_{n+1}-p\right\rangle \\
& \leq\left[\lambda_{n}\left(\alpha_{n} \gamma \rho\left\|x_{n}-p\right\|+\left(1-\alpha_{n}\right) \gamma\left\|x_{n}-p\right\|\right)+\left(1-\lambda_{n} \tau\right)\left\|y_{n}-p\right\|\right]^{2} \\
& +2 \lambda_{n} \alpha_{n}\left\langle(\gamma V p-\mu F p), x_{n+1}-p\right\rangle+2\left(1-\alpha_{n}\right) \lambda_{n}\left|(\gamma S p-\mu F p), x_{n+1}-p\right\rangle \\
& =\left[\lambda_{n}\left(1-\alpha_{n}(1-\rho)\right) \gamma\left\|x_{n}-p\right\|+\left(1-\lambda_{n} \tau\right)\left\|y_{n}-p\right\|\right]^{2} \\
& +2 \lambda_{n} \alpha_{n}\left\langle(\gamma V p-\mu F p), x_{n+1}-p\right\rangle+2 \lambda_{n}\left(1-\alpha_{n}\right)\left\langle(\gamma S p-\mu F p), x_{n+1}-p\right\rangle \\
& \leq\left[\lambda_{n} \gamma\left\|x_{n}-p\right\|+\left(1-\lambda_{n} \tau\right)\left\|y_{n}-p\right\|\right]^{2} \\
& +2 \lambda_{n} \alpha_{n}\left\langle(\gamma V p-\mu F p), x_{n+1}-p\right\rangle+2 \lambda_{n}\left(1-\alpha_{n}\right)\left\langle(\gamma S p-\mu F p), x_{n+1}-p\right\rangle \\
& =\left[\lambda_{n} \tau \cdot \frac{\gamma}{\tau}\left\|x_{n}-p\right\|+\left(1-\lambda_{n} \tau\right)\left\|y_{n}-p\right\|\right]^{2} \\
& +2 \lambda_{n} \alpha_{n}\left\langle(\gamma V p-\mu F p), x_{n+1}-p\right\rangle+2 \lambda_{n}\left(1-\alpha_{n}\right)\left\langle(\gamma S p-\mu F p), x_{n+1}-p\right\rangle \\
& \leq \lambda_{n} \frac{\gamma^{2}}{\tau}\left\|x_{n}-p\right\|+\left(1-\lambda_{n} \tau\right)\left\|y_{n}-p\right\|^{2} \\
& +2 \lambda_{n} \alpha_{n}\left\langle(\gamma V p-\mu F p), x_{n+1}-p\right\rangle+2 \lambda_{n}\left(1-\alpha_{n}\right)\left\langle(\gamma S p-\mu F p), x_{n+1}-p\right\rangle \\
& \leq \lambda_{n} \cdot \frac{\gamma^{2}}{\tau}\left\|x_{n}-p\right\|+\left(1-\lambda_{n} \tau\right)\left[\left\|x_{n}-p\right\|^{2}-\frac{\beta_{n}}{1-\beta_{n}}\left\|y_{n}-x_{n}\right\|^{2}\right] \\
& +2 \lambda_{n} \alpha_{n}\left\langle(\gamma V p-\mu F p), x_{n+1}-p\right\rangle+2 \lambda_{n}\left(1-\alpha_{n}\right)\left\langle(\gamma S p-\mu F p), x_{n+1}-p\right\rangle \\
& =\left(1-\lambda_{n} \frac{\tau^{2}-\gamma^{2}}{\tau}\right)\left\|x_{n}-p\right\|^{2}-\frac{\beta_{n}\left(1-\lambda_{n} \tau\right)}{1-\beta_{n}}\left\|y_{n}-x_{n}\right\|^{2} \\
& +2 \lambda_{n} \alpha_{n}\left\langle(\gamma V p-\mu F p), x_{n+1}-p\right\rangle+2 \lambda_{n}\left(1-\alpha_{n}\right)\left\langle(\gamma S p-\mu F p), x_{n+1}-p\right\rangle \\
& \leq\left\|x_{n}-p\right\|^{2}-\frac{\beta_{n}\left(1-\lambda_{n} \tau\right)}{1-\beta_{n}}\left\|y_{n}-x_{n}\right\|^{2} \\
& +2 \lambda_{n} \alpha_{n}\|\gamma V p-\mu F p\|\left\|x_{n+1}-p\right\|+2 \lambda_{n}\|\gamma S p-\mu F p\|\left\|x_{n+1}-p\right\|,
\end{aligned}
$$


which together with $\left\{\beta_{n}\right\} \subset[a, b] \subset(0,1)$, immediately yields

$$
\begin{aligned}
& \frac{a\left(1-\lambda_{n} \tau\right)}{1-a}\left\|y_{n}-x_{n}\right\|^{2} \\
& \leq \frac{\beta_{n}\left(1-\lambda_{n} \tau\right)}{1-\beta_{n}}\left\|y_{n}-x_{n}\right\|^{2} \\
& \leq\left\|x_{n}-p\right\|^{2}-\left\|x_{n+1}-p\right\|^{2}+2 \lambda_{n} \alpha_{n}\|\gamma V p-\mu F p\|\left\|x_{n+1}-p\right\| \\
& \quad+2 \lambda_{n}\|\gamma S p-\mu F p\|\left\|x_{n+1}-p\right\| \\
& \leq\left\|x_{n}-x_{n+1}\right\|\left(\left\|x_{n}-p\right\|+\left\|x_{n+1}-p\right\|\right) \\
&+2 \lambda_{n} \alpha_{n}\|\gamma V p-\mu F p\|\left\|x_{n+1}-p\right\|+2 \lambda_{n}\|\gamma S p-\mu F p\|\left\|x_{n+1}-p\right\| .
\end{aligned}
$$

Since $\lambda_{n} \rightarrow 0, \alpha_{n} \rightarrow 0,\left\|x_{n+1}-x_{n}\right\| \rightarrow 0$, and $\left\{x_{n}\right\}$ is bounded, we have

$$
\lim _{n \rightarrow \infty}\left\|y_{n}-x_{n}\right\|=0
$$

Observe that

$$
\begin{aligned}
\left\|\Delta_{n}^{k} x_{n}-p\right\|^{2} & =\left\|T_{r_{k, n}}^{\left(\Theta_{k}, \varphi_{k}\right)}\left(I-r_{k, n} A_{k}\right) \Delta_{n}^{k-1} x_{n}-T_{r_{k, n}}^{\left(\Theta_{k}, \varphi_{k}\right)}\left(I-r_{k, n} A_{k}\right) p\right\|^{2} \\
& \leq\left\|\left(I-r_{k, n} A_{k}\right) \Delta_{n}^{k-1} x_{n}-\left(I-r_{k, n} A_{k}\right) p\right\|^{2} \\
& \leq\left\|\Delta_{n}^{k-1} x_{n}-p\right\|^{2}+r_{k, n}\left(r_{k, n}-2 \mu_{k}\right)\left\|A_{k} \Delta_{n}^{k-1} x_{n}-A_{k} p\right\|^{2} \\
& \leq\left\|x_{n}-p\right\|^{2}+r_{k, n}\left(r_{k, n}-2 \mu_{k}\right)\left\|A_{k} \Delta_{n}^{k-1} x_{n}-A_{k} p\right\|^{2}
\end{aligned}
$$

and

$$
\begin{aligned}
\left\|\Lambda_{n}^{i} u_{n}-p\right\|^{2} & =\left\|J_{R_{i}, \lambda_{i, n}}\left(I-\lambda_{i, n} B_{i}\right) \Lambda_{n}^{i-1} u_{n}-J_{R_{i}, \lambda_{i, n}}\left(I-\lambda_{i, n} B_{i}\right) p\right\|^{2} \\
& \leq\left\|\left(I-\lambda_{i, n} B_{i}\right) \Lambda_{n}^{i-1} u_{n}-\left(I-\lambda_{i, n} B_{i}\right) p\right\|^{2} \\
& \leq\left\|\Lambda_{n}^{i-1} u_{n}-p\right\|^{2}+\lambda_{i, n}\left(\lambda_{i, n}-2 \eta_{i}\right)\left\|B_{i} \Lambda_{n}^{i-1} u_{n}-B_{i} p\right\|^{2} \\
& \leq\left\|u_{n}-p\right\|^{2}+\lambda_{i, n}\left(\lambda_{i, n}-2 \eta_{i}\right)\left\|B_{i} \Lambda_{n}^{i-1} u_{n}-B_{i} p\right\|^{2} \\
& \leq\left\|x_{n}-p\right\|^{2}+\lambda_{i, n}\left(\lambda_{i, n}-2 \eta_{i}\right)\left\|B_{i} \Lambda_{n}^{i-1} u_{n}-B_{i} p\right\|^{2}
\end{aligned}
$$

for $i \in\{1,2, \ldots, N\}$ and $k \in\{1,2, \ldots, M\}$. Combining (3.14), (3.17), and (3.18), we get

$$
\begin{aligned}
\left\|y_{n}-p\right\|^{2} \leq & \beta_{n}\left\|x_{n}-p\right\|^{2}+\left(1-\beta_{n}\right)\left\|v_{n}-p\right\|^{2}-\frac{\beta_{n}}{1-\beta_{n}}\left\|y_{n}-x_{n}\right\|^{2} \\
\leq & \beta_{n}\left\|x_{n}-p\right\|^{2}+\left(1-\beta_{n}\right)\left\|v_{n}-p\right\|^{2} \\
\leq & \beta_{n}\left\|x_{n}-p\right\|^{2}+\left(1-\beta_{n}\right)\left\|\Lambda_{n}^{i} u_{n}-p\right\|^{2} \\
\leq & \beta_{n}\left\|x_{n}-p\right\|^{2}+\left(1-\beta_{n}\right)\left[\left\|u_{n}-p\right\|^{2}+\lambda_{i, n}\left(\lambda_{i, n}-2 \eta_{i}\right)\left\|B_{i} \Lambda_{n}^{i-1} u_{n}-B_{i} p\right\|^{2}\right] \\
\leq & \beta_{n}\left\|x_{n}-p\right\|^{2}+\left(1-\beta_{n}\right)\left[\left\|\Delta_{n}^{k} x_{n}-p\right\|^{2}+\lambda_{i, n}\left(\lambda_{i, n}-2 \eta_{i}\right)\left\|B_{i} \Lambda_{n}^{i-1} u_{n}-B_{i} p\right\|^{2}\right] \\
\leq & \beta_{n}\left\|x_{n}-p\right\|^{2}+\left(1-\beta_{n}\right)\left[\left\|x_{n}-p\right\|^{2}+r_{k, n}\left(r_{k, n}-2 \mu_{k}\right)\left\|A_{k} \Delta_{n}^{k-1} x_{n}-A_{k} p\right\|^{2}\right. \\
& \left.+\lambda_{i, n}\left(\lambda_{i, n}-2 \eta_{i}\right)\left\|B_{i} \Lambda_{n}^{i-1} u_{n}-B_{i} p\right\|^{2}\right]
\end{aligned}
$$




$$
\begin{aligned}
= & \left\|x_{n}-p\right\|^{2}+\left(1-\beta_{n}\right)\left[r_{k, n}\left(r_{k, n}-2 \mu_{k}\right)\left\|A_{k} \Delta_{n}^{k-1} x_{n}-A_{k} p\right\|^{2}\right. \\
& \left.+\lambda_{i, n}\left(\lambda_{i, n}-2 \eta_{i}\right)\left\|B_{i} \Lambda_{n}^{i-1} u_{n}-B_{i} p\right\|^{2}\right],
\end{aligned}
$$

which immediately leads to

$$
\begin{aligned}
& \left(1-\beta_{n}\right)\left[r_{k, n}\left(2 \mu_{k}-r_{k, n}\right)\left\|A_{k} \Delta_{n}^{k-1} x_{n}-A_{k} p\right\|^{2}+\lambda_{i, n}\left(2 \eta_{i}-\lambda_{i, n}\right)\left\|B_{i} \Lambda_{n}^{i-1} u_{n}-B_{i} p\right\|^{2}\right] \\
& \quad \leq\left\|x_{n}-p\right\|^{2}-\left\|y_{n}-p\right\|^{2} \\
& \quad \leq\left\|x_{n}-y_{n}\right\|\left(\left\|x_{n}-p\right\|+\left\|y_{n}-p\right\|\right) .
\end{aligned}
$$

Since $\left\|x_{n}-y_{n}\right\| \rightarrow 0,\left\{\beta_{n}\right\} \subset[a, b] \subset(0,1),\left\{\lambda_{i, n}\right\} \subset\left[a_{i}, b_{i}\right] \subset\left(0,2 \eta_{i}\right),\left\{r_{k, n}\right\} \subset\left[c_{k}, d_{k}\right] \subset$ $\left(0,2 \mu_{k}\right), i \in\{1,2, \ldots, N\}, k \in\{1,2, \ldots, M\}$, and $\left\{x_{n}\right\},\left\{y_{n}\right\}$ are bounded sequences, we have

$$
\lim _{n \rightarrow \infty}\left\|A_{k} \Delta_{n}^{k-1} x_{n}-A_{k} p\right\|=0 \quad \text { and } \quad \lim _{n \rightarrow \infty}\left\|B_{i} \Lambda_{n}^{i-1} u_{n}-B_{i} p\right\|=0
$$

for all $k \in\{1,2, \ldots, M\}$ and $i \in\{1,2, \ldots, N\}$.

Furthermore, by Proposition 2.2(ii) and Lemma 2.2(a), we have

$$
\begin{aligned}
&\left\|\Delta_{n}^{k} x_{n}-p\right\|^{2} \\
&=\left\|T_{r_{k, n}}^{\left(\Theta_{k}, \varphi_{k}\right)}\left(I-r_{k, n} A_{k}\right) \Delta_{n}^{k-1} x_{n}-T_{r_{k, n}}^{\left(\Theta_{k}, \varphi_{k}\right)}\left(I-r_{k, n} A_{k}\right) p\right\|^{2} \\
& \leq\left\langle\left(I-r_{k, n} A_{k}\right) \Delta_{n}^{k-1} x_{n}-\left(I-r_{k, n} A_{k}\right) p, \Delta_{n}^{k} x_{n}-p\right\rangle \\
&= \frac{1}{2}\left(\left\|\left(I-r_{k, n} A_{k}\right) \Delta_{n}^{k-1} x_{n}-\left(I-r_{k, n} A_{k}\right) p\right\|^{2}+\left\|\Delta_{n}^{k} x_{n}-p\right\|^{2}\right. \\
&\left.\quad-\left\|\left(I-r_{k, n} A_{k}\right) \Delta_{n}^{k-1} x_{n}-\left(I-r_{k, n} A_{k}\right) p-\left(\Delta_{n}^{k} x_{n}-p\right)\right\|^{2}\right) \\
& \leq \frac{1}{2}\left(\left\|\Delta_{n}^{k-1} x_{n}-p\right\|^{2}+\left\|\Delta_{n}^{k} x_{n}-p\right\|^{2}-\left\|\Delta_{n}^{k-1} x_{n}-\Delta_{n}^{k} x_{n}-r_{k, n}\left(A_{k} \Delta_{n}^{k-1} x_{n}-A_{k} p\right)\right\|^{2}\right),
\end{aligned}
$$

which implies that

$$
\begin{aligned}
\left\|\Delta_{n}^{k} x_{n}-p\right\|^{2} \\
\leq \\
\leq\left\|\Delta_{n}^{k-1} x_{n}-p\right\|^{2}-\left\|\Delta_{n}^{k-1} x_{n}-\Delta_{n}^{k} x_{n}-r_{k, n}\left(A_{k} \Delta_{n}^{k-1} x_{n}-A_{k} p\right)\right\|^{2} \\
=\left\|\Delta_{n}^{k-1} x_{n}-p\right\|^{2}-\left\|\Delta_{n}^{k-1} x_{n}-\Delta_{n}^{k} x_{n}\right\|^{2}-r_{k, n}^{2}\left\|A_{k} \Delta_{n}^{k-1} x_{n}-A_{k} p\right\|^{2} \\
\quad+2 r_{k, n}\left(\Delta_{n}^{k-1} x_{n}-\Delta_{n}^{k} x_{n}, A_{k} \Delta_{n}^{k-1} x_{n}-A_{k} p\right\rangle \\
\leq\left\|\Delta_{n}^{k-1} x_{n}-p\right\|^{2}-\left\|\Delta_{n}^{k-1} x_{n}-\Delta_{n}^{k} x_{n}\right\|^{2}+2 r_{k, n}\left\|\Delta_{n}^{k-1} x_{n}-\Delta_{n}^{k} x_{n}\right\|\left\|A_{k} \Delta_{n}^{k-1} x_{n}-A_{k} p\right\| \\
\leq\left\|x_{n}-p\right\|^{2}-\left\|\Delta_{n}^{k-1} x_{n}-\Delta_{n}^{k} x_{n}\right\|^{2}+2 r_{k, n}\left\|\Delta_{n}^{k-1} x_{n}-\Delta_{n}^{k} x_{n}\right\|\left\|A_{k} \Delta_{n}^{k-1} x_{n}-A_{k} p\right\| . \text { (3.20) }
\end{aligned}
$$

By Lemma 2.2(a) and Lemma 2.10, we obtain

$$
\begin{aligned}
& \left\|\Lambda_{n}^{i} u_{n}-p\right\|^{2} \\
& \quad=\left\|J_{R_{i}, \lambda_{i, n}}\left(I-\lambda_{i, n} B_{i}\right) \Lambda_{n}^{i-1} u_{n}-J_{R_{i}, \lambda_{i, n}}\left(I-\lambda_{i, n} B_{i}\right) p\right\|^{2} \\
& \quad \leq\left\langle\left(I-\lambda_{i, n} B_{i}\right) \Lambda_{n}^{i-1} u_{n}-\left(I-\lambda_{i, n} B_{i}\right) p, \Lambda_{n}^{i} u_{n}-p\right\rangle \\
& \quad=\frac{1}{2}\left(\left\|\left(I-\lambda_{i, n} B_{i}\right) \Lambda_{n}^{i-1} u_{n}-\left(I-\lambda_{i, n} B_{i}\right) p\right\|^{2}+\left\|\Lambda_{n}^{i} u_{n}-p\right\|^{2}\right.
\end{aligned}
$$




$$
\begin{aligned}
& \left.-\left\|\left(I-\lambda_{i, n} B_{i}\right) \Lambda_{n}^{i-1} u_{n}-\left(I-\lambda_{i, n} B_{i}\right) p-\left(\Lambda_{n}^{i} u_{n}-p\right)\right\|^{2}\right) \\
\leq & \frac{1}{2}\left(\left\|\Lambda_{n}^{i-1} u_{n}-p\right\|^{2}+\left\|\Lambda_{n}^{i} u_{n}-p\right\|^{2}-\left\|\Lambda_{n}^{i-1} u_{n}-\Lambda_{n}^{i} u_{n}-\lambda_{i, n}\left(B_{i} \Lambda_{n}^{i-1} u_{n}-B_{i} p\right)\right\|^{2}\right) \\
\leq & \frac{1}{2}\left(\left\|u_{n}-p\right\|^{2}+\left\|\Lambda_{n}^{i} u_{n}-p\right\|^{2}-\left\|\Lambda_{n}^{i-1} u_{n}-\Lambda_{n}^{i} u_{n}-\lambda_{i, n}\left(B_{i} \Lambda_{n}^{i-1} u_{n}-B_{i} p\right)\right\|^{2}\right) \\
\leq & \frac{1}{2}\left(\left\|x_{n}-p\right\|^{2}+\left\|\Lambda_{n}^{i} u_{n}-p\right\|^{2}-\left\|\Lambda_{n}^{i-1} u_{n}-\Lambda_{n}^{i} u_{n}-\lambda_{i, n}\left(B_{i} \Lambda_{n}^{i-1} u_{n}-B_{i} p\right)\right\|^{2}\right),
\end{aligned}
$$

which immediately leads to

$$
\begin{aligned}
& \left\|\Lambda_{n}^{i} u_{n}-p\right\|^{2} \\
& \quad \leq\left\|x_{n}-p\right\|^{2}-\left\|\Lambda_{n}^{i-1} u_{n}-\Lambda_{n}^{i} u_{n}-\lambda_{i, n}\left(B_{i} \Lambda_{n}^{i-1} u_{n}-B_{i} p\right)\right\|^{2} \\
& =\left\|x_{n}-p\right\|^{2}-\left\|\Lambda_{n}^{i-1} u_{n}-\Lambda_{n}^{k} u_{n}\right\|^{2}-\lambda_{i, n}^{2}\left\|B_{i} \Lambda_{n}^{i-1} u_{n}-B_{i} p\right\|^{2} \\
& \quad+2 \lambda_{i, n}\left\langle\Lambda_{n}^{i-1} u_{n}-\Lambda_{n}^{i} u_{n}, B_{i} \Lambda_{n}^{i-1} u_{n}-B_{i} p\right\rangle \\
& \leq\left\|x_{n}-p\right\|^{2}-\left\|\Lambda_{n}^{i-1} u_{n}-\Lambda_{n}^{i} u_{n}\right\|^{2}+2 \lambda_{i, n}\left\|\Lambda_{n}^{i-1} u_{n}-\Lambda_{n}^{i} u_{n}\right\|\left\|B_{i} \Lambda_{n}^{i-1} u_{n}-B_{i} p\right\| .
\end{aligned}
$$

Combining (3.14) and (3.21), we conclude that

$$
\begin{aligned}
\left\|y_{n}-p\right\|^{2} \leq & \beta_{n}\left\|x_{n}-p\right\|^{2}+\left(1-\beta_{n}\right)\left\|v_{n}-p\right\|^{2}-\frac{\beta_{n}}{1-\beta_{n}}\left\|y_{n}-x_{n}\right\|^{2} \\
\leq & \beta_{n}\left\|x_{n}-p\right\|^{2}+\left(1-\beta_{n}\right)\left\|v_{n}-p\right\|^{2} \\
\leq & \beta_{n}\left\|x_{n}-p\right\|^{2}+\left(1-\beta_{n}\right)\left\|\Lambda_{n}^{i} u_{n}-p\right\|^{2} \\
\leq & \beta_{n}\left\|x_{n}-p\right\|^{2}+\left(1-\beta_{n}\right)\left[\left\|x_{n}-p\right\|^{2}-\left\|\Lambda_{n}^{i-1} u_{n}-\Lambda_{n}^{i} u_{n}\right\|^{2}\right. \\
& \left.+2 \lambda_{i, n}\left\|\Lambda_{n}^{i-1} u_{n}-\Lambda_{n}^{i} u_{n}\right\|\left\|B_{i} \Lambda_{n}^{i-1} u_{n}-B_{i} p\right\|\right] \\
\leq & \left\|x_{n}-p\right\|^{2}-\left(1-\beta_{n}\right)\left\|\Lambda_{n}^{i-1} u_{n}-\Lambda_{n}^{i} u_{n}\right\|^{2} \\
& +2 \lambda_{i, n}\left\|\Lambda_{n}^{i-1} u_{n}-\Lambda_{n}^{i} u_{n}\right\|\left\|B_{i} \Lambda_{n}^{i-1} u_{n}-B_{i} p\right\|,
\end{aligned}
$$

which yields

$$
\begin{aligned}
& \left(1-\beta_{n}\right)\left\|\Lambda_{n}^{i-1} u_{n}-\Lambda_{n}^{i} u_{n}\right\|^{2} \\
& \quad \leq\left\|x_{n}-p\right\|^{2}-\left\|y_{n}-p\right\|^{2}+2 \lambda_{i, n}\left\|\Lambda_{n}^{i-1} u_{n}-\Lambda_{n}^{i} u_{n}\right\|\left\|B_{i} \Lambda_{n}^{i-1} u_{n}-B_{i} p\right\| \\
& \quad \leq\left\|x_{n}-y_{n}\right\|\left(\left\|x_{n}-p\right\|+\left\|y_{n}-p\right\|\right)+2 \lambda_{i, n}\left\|\Lambda_{n}^{i-1} u_{n}-\Lambda_{n}^{i} u_{n}\right\|\left\|B_{i} \Lambda_{n}^{i-1} u_{n}-B_{i} p\right\| .
\end{aligned}
$$

Since $\left\{\beta_{n}\right\} \subset[a, b] \subset(0,1),\left\{\lambda_{i, n}\right\} \subset\left[a_{i}, b_{i}\right] \subset\left(0,2 \eta_{i}\right), i=1,2, \ldots, N$, and $\left\{u_{n}\right\},\left\{x_{n}\right\}$, and $\left\{y_{n}\right\}$ are bounded sequences, we deduce from (3.19) and $\left\|x_{n}-y_{n}\right\| \rightarrow 0$ that

$$
\lim _{n \rightarrow \infty}\left\|\Lambda_{n}^{i-1} u_{n}-\Lambda_{n}^{i} u_{n}\right\|=0, \quad \forall i \in\{1,2, \ldots, N\}
$$

Also, combining (3.3), (3.14), and (3.20), we deduce that

$$
\begin{aligned}
\left\|y_{n}-p\right\|^{2} & \leq \beta_{n}\left\|x_{n}-p\right\|^{2}+\left(1-\beta_{n}\right)\left\|v_{n}-p\right\|^{2}-\frac{\beta_{n}}{1-\beta_{n}}\left\|y_{n}-x_{n}\right\|^{2} \\
& \leq \beta_{n}\left\|x_{n}-p\right\|^{2}+\left(1-\beta_{n}\right)\left\|v_{n}-p\right\|^{2}
\end{aligned}
$$




$$
\begin{aligned}
\leq & \beta_{n}\left\|x_{n}-p\right\|^{2}+\left(1-\beta_{n}\right)\left\|u_{n}-p\right\|^{2} \\
\leq & \beta_{n}\left\|x_{n}-p\right\|^{2}+\left(1-\beta_{n}\right)\left\|\Delta_{n}^{k} x_{n}-p\right\|^{2} \\
\leq & \beta_{n}\left\|x_{n}-p\right\|^{2}+\left(1-\beta_{n}\right)\left[\left\|x_{n}-p\right\|^{2}-\left\|\Delta_{n}^{k-1} x_{n}-\Delta_{n}^{k} x_{n}\right\|^{2}\right. \\
& \left.+2 r_{k, n}\left\|\Delta_{n}^{k-1} x_{n}-\Delta_{n}^{k} x_{n}\right\|\left\|A_{k} \Delta_{n}^{k-1} x_{n}-A_{k} p\right\|\right] \\
\leq & \left\|x_{n}-p\right\|^{2}-\left(1-\beta_{n}\right)\left\|\Delta_{n}^{k-1} x_{n}-\Delta_{n}^{k} x_{n}\right\|^{2} \\
& +2 r_{k, n}\left\|\Delta_{n}^{k-1} x_{n}-\Delta_{n}^{k} x_{n}\right\|\left\|A_{k} \Delta_{n}^{k-1} x_{n}-A_{k} p\right\|,
\end{aligned}
$$

which yields

$$
\begin{aligned}
& \left(1-\beta_{n}\right)\left\|\Delta_{n}^{k-1} x_{n}-\Delta_{n}^{k} x_{n}\right\|^{2} \\
& \quad \leq\left\|x_{n}-p\right\|^{2}-\left\|y_{n}-p\right\|^{2}+2 r_{k, n}\left\|\Delta_{n}^{k-1} x_{n}-\Delta_{n}^{k} x_{n}\right\|\left\|A_{k} \Delta_{n}^{k-1} x_{n}-A_{k} p\right\| \\
& \quad \leq\left\|x_{n}-y_{n}\right\|\left(\left\|x_{n}-p\right\|+\left\|y_{n}-p\right\|\right)+2 r_{k, n}\left\|\Delta_{n}^{k-1} x_{n}-\Delta_{n}^{k} x_{n}\right\|\left\|A_{k} \Delta_{n}^{k-1} x_{n}-A_{k} p\right\| .
\end{aligned}
$$

Since $\left\{\beta_{n}\right\} \subset[a, b] \subset(0,1),\left\{r_{k, n}\right\} \subset\left[c_{k}, d_{k}\right] \subset\left(0,2 \mu_{k}\right)$ for $k=1,2, \ldots, M$, and $\left\{x_{n}\right\},\left\{y_{n}\right\}$ are bounded sequences, we deduce from (3.19) and $\left\|x_{n}-y_{n}\right\| \rightarrow 0$ that

$$
\lim _{n \rightarrow \infty}\left\|\Delta_{n}^{k-1} x_{n}-\Delta_{n}^{k} x_{n}\right\|=0, \quad \forall k \in\{1,2, \ldots, M\}
$$

Hence, from (3.22) and (3.23), we get

$$
\begin{aligned}
\left\|x_{n}-u_{n}\right\| & =\left\|\Delta_{n}^{0} x_{n}-\Delta_{n}^{M} x_{n}\right\| \\
& \leq\left\|\Delta_{n}^{0} x_{n}-\Delta_{n}^{1} x_{n}\right\|+\left\|\Delta_{n}^{1} x_{n}-\Delta_{n}^{2} x_{n}\right\|+\cdots+\left\|\Delta_{n}^{M-1} x_{n}-\Delta_{n}^{M} x_{n}\right\| \\
& \rightarrow 0 \quad \text { as } n \rightarrow \infty
\end{aligned}
$$

and

$$
\begin{aligned}
\left\|u_{n}-v_{n}\right\| & =\left\|\Lambda_{n}^{0} u_{n}-\Lambda_{n}^{N} u_{n}\right\| \\
& \leq\left\|\Lambda_{n}^{0} u_{n}-\Lambda_{n}^{1} u_{n}\right\|+\left\|\Lambda_{n}^{1} u_{n}-\Lambda_{n}^{2} u_{n}\right\|+\cdots+\left\|\Lambda_{n}^{N-1} u_{n}-\Lambda_{n}^{N} u_{n}\right\| \\
& \rightarrow 0 \quad \text { as } n \rightarrow \infty
\end{aligned}
$$

respectively. Thus, from (3.24) and (3.25), we obtain

$$
\begin{aligned}
\left\|x_{n}-v_{n}\right\| & \leq\left\|x_{n}-u_{n}\right\|+\left\|u_{n}-v_{n}\right\| \\
& \rightarrow 0 \quad \text { as } n \rightarrow \infty .
\end{aligned}
$$

On the other hand, for simplicity, we write $\tilde{p}=P_{C}\left(I-v_{2} F_{2}\right) p, \tilde{v}_{n}=P_{C}\left(I-v_{2} F_{2}\right) v_{n}$, and $k_{n}=G v_{n}=P_{C}\left(I-v_{1} F_{1}\right) \tilde{v}_{n}$ for all $n \geq 1$. Then

$$
p=G p=P_{C}\left(I-v_{1} F_{1}\right) \tilde{p}=P_{C}\left(I-v_{1} F_{1}\right) P_{C}\left(I-v_{2} F_{2}\right) p .
$$


We now show that $\lim _{n \rightarrow \infty}\left\|G v_{n}-v_{n}\right\|=0$, that is, $\lim _{n \rightarrow \infty}\left\|k_{n}-v_{n}\right\|=0$. As a matter of fact, for $p \in \Omega$, it follows from (3.4), (3.5), and (3.14) that

$$
\begin{aligned}
\left\|y_{n}-p\right\|^{2} \leq & \beta_{n}\left\|x_{n}-p\right\|^{2}+\left(1-\beta_{n}\right)\left\|G v_{n}-p\right\|^{2}-\frac{\beta_{n}}{1-\beta_{n}}\left\|y_{n}-x_{n}\right\|^{2} \\
\leq & \beta_{n}\left\|x_{n}-p\right\|^{2}+\left(1-\beta_{n}\right)\left\|G v_{n}-p\right\|^{2} \\
= & \beta_{n}\left\|x_{n}-p\right\|^{2}+\left(1-\beta_{n}\right)\left\|k_{n}-p\right\|^{2} \\
\leq & \beta_{n}\left\|x_{n}-p\right\|^{2}+\left(1-\beta_{n}\right)\left[\left\|\tilde{v}_{n}-\tilde{p}\right\|^{2}+v_{1}\left(v_{1}-2 \zeta_{1}\right)\left\|F_{1} \tilde{v}_{n}-F_{1} \tilde{p}\right\|^{2}\right] \\
\leq & \beta_{n}\left\|x_{n}-p\right\|^{2}+\left(1-\beta_{n}\right)\left[\left\|v_{n}-p\right\|^{2}+v_{2}\left(v_{2}-2 \zeta_{2}\right)\left\|F_{2} v_{n}-F_{2} p\right\|^{2}\right. \\
& \left.+v_{1}\left(v_{1}-2 \zeta_{1}\right)\left\|F_{1} \tilde{v}_{n}-F_{1} \tilde{p}\right\|^{2}\right] \\
\leq & \beta_{n}\left\|x_{n}-p\right\|^{2}+\left(1-\beta_{n}\right)\left[\left\|x_{n}-p\right\|^{2}+v_{2}\left(v_{2}-2 \zeta_{2}\right)\left\|F_{2} v_{n}-F_{2} p\right\|^{2}\right. \\
& \left.+v_{1}\left(v_{1}-2 \zeta_{1}\right)\left\|F_{1} \tilde{v}_{n}-F_{1} \tilde{p}\right\|^{2}\right] \\
= & \left\|x_{n}-p\right\|^{2}+\left(1-\beta_{n}\right)\left[v_{2}\left(v_{2}-2 \zeta_{2}\right)\left\|F_{2} v_{n}-F_{2} p\right\|^{2}\right. \\
& \left.+v_{1}\left(v_{1}-2 \zeta_{1}\right)\left\|F_{1} \tilde{v}_{n}-F_{1} \tilde{p}\right\|^{2}\right],
\end{aligned}
$$

which immediately yields

$$
\begin{aligned}
& \left(1-\beta_{n}\right)\left[v_{2}\left(2 \zeta_{2}-v_{2}\right)\left\|F_{2} v_{n}-F_{2} p\right\|^{2}+v_{1}\left(2 \zeta_{1}-v_{1}\right)\left\|F_{1} \tilde{v}_{n}-F_{1} \tilde{p}\right\|^{2}\right] \\
& \quad \leq\left\|x_{n}-p\right\|^{2}-\left\|y_{n}-p\right\|^{2} \\
& \quad \leq\left\|x_{n}-y_{n}\right\|\left(\left\|x_{n}-p\right\|+\left\|y_{n}-p\right\|\right) .
\end{aligned}
$$

Since $\left\|x_{n}-y_{n}\right\| \rightarrow 0,\left\{\beta_{n}\right\} \subset[a, b] \subset(0,1), v_{j} \in\left(0,2 \zeta_{j}\right), j=1,2$, and $\left\{x_{n}\right\},\left\{y_{n}\right\}$ are bounded sequences, we have

$$
\lim _{n \rightarrow \infty}\left\|F_{2} v_{n}-F_{2} p\right\|=0 \quad \text { and } \quad \lim _{n \rightarrow \infty}\left\|F_{1} \tilde{v}_{n}-F_{1} \tilde{p}\right\|=0 .
$$

Also, in terms of the firm nonexpansivity of $P_{C}$ and the $\zeta_{j}$-inverse-strong monotonicity of $F_{j}$ for $j=1,2$, we obtain from $v_{j} \in\left(0,2 \zeta_{j}\right), j=1,2$, and (3.5)

$$
\begin{aligned}
\left\|\tilde{v}_{n}-\tilde{p}\right\|^{2}= & \left\|P_{C}\left(I-v_{2} F_{2}\right) v_{n}-P_{C}\left(I-v_{2} F_{2}\right) p\right\|^{2} \\
\leq & \left\langle\left(I-v_{2} F_{2}\right) v_{n}-\left(I-v_{2} F_{2}\right) p, \tilde{v}_{n}-\tilde{p}\right\rangle \\
= & \frac{1}{2}\left[\left\|\left(I-v_{2} F_{2}\right) v_{n}-\left(I-v_{2} F_{2}\right) p\right\|^{2}+\left\|\tilde{v}_{n}-\tilde{p}\right\|^{2}\right. \\
& \left.-\left\|\left(I-v_{2} F_{2}\right) v_{n}-\left(I-v_{2} F_{2}\right) p-\left(\tilde{v}_{n}-\tilde{p}\right)\right\|^{2}\right] \\
\leq & \frac{1}{2}\left[\left\|v_{n}-p\right\|^{2}+\left\|\tilde{v}_{n}-\tilde{p}\right\|^{2}\right. \\
& \left.-\left\|\left(v_{n}-\tilde{v}_{n}\right)-v_{2}\left(F_{2} v_{n}-F_{2} p\right)-(p-\tilde{p})\right\|^{2}\right] \\
= & \frac{1}{2}\left[\left\|v_{n}-p\right\|^{2}+\left\|\tilde{v}_{n}-\tilde{p}\right\|^{2}-\left\|\left(v_{n}-\tilde{v}_{n}\right)-(p-\tilde{p})\right\|^{2}\right. \\
& \left.+2 v_{2}\left(\left(v_{n}-\tilde{v}_{n}\right)-(p-\tilde{p}), F_{2} v_{n}-F_{2} p\right\rangle-v_{2}^{2}\left\|F_{2} v_{n}-F_{2} p\right\|^{2}\right]
\end{aligned}
$$


and

$$
\begin{aligned}
\left\|k_{n}-p\right\|^{2}= & \left\|P_{C}\left(I-v_{1} F_{1}\right) \tilde{v}_{n}-P_{C}\left(I-v_{1} F_{1}\right) \tilde{p}\right\|^{2} \\
\leq & \left\langle\left(I-v_{1} F_{1}\right) \tilde{v}_{n}-\left(I-v_{1} F_{1}\right) \tilde{p}, k_{n}-p\right\rangle \\
= & \frac{1}{2}\left[\left\|\left(I-v_{1} F_{1}\right) \tilde{v}_{n}-\left(I-v_{1} F_{1}\right) \tilde{p}\right\|^{2}+\left\|k_{n}-p\right\|^{2}\right. \\
& \left.-\left\|\left(I-v_{1} F_{1}\right) \tilde{v}_{n}-\left(I-v_{1} F_{1}\right) \tilde{p}-\left(k_{n}-p\right)\right\|^{2}\right] \\
\leq & \frac{1}{2}\left[\left\|\tilde{v}_{n}-\tilde{p}\right\|^{2}+\left\|k_{n}-p\right\|^{2}-\left\|\left(\tilde{v}_{n}-k_{n}\right)+(p-\tilde{p})\right\|^{2}\right. \\
& \left.+2 v_{1}\left\langle F_{1} \tilde{v}_{n}-F_{1} \tilde{p},\left(\tilde{v}_{n}-k_{n}\right)+(p-\tilde{p})\right\rangle-v_{1}^{2}\left\|F_{1} \tilde{v}_{n}-F_{1} \tilde{p}\right\|^{2}\right] \\
\leq & \frac{1}{2}\left[\left\|v_{n}-p\right\|^{2}+\left\|w_{n}-p\right\|^{2}-\left\|\left(\tilde{v}_{n}-k_{n}\right)+(p-\tilde{p})\right\|^{2}\right. \\
& \left.+2 v_{1}\left\langle F_{1} \tilde{v}_{n}-F_{1} \tilde{p},\left(\tilde{v}_{n}-k_{n}\right)+(p-\tilde{p})\right\rangle\right] .
\end{aligned}
$$

Thus, we have

$$
\begin{aligned}
\left\|\tilde{v}_{n}-\tilde{p}\right\|^{2} \leq & \left\|v_{n}-p\right\|^{2}-\left\|\left(v_{n}-\tilde{v}_{n}\right)-(p-\tilde{p})\right\|^{2} \\
& +2 v_{2}\left(\left(v_{n}-\tilde{v}_{n}\right)-(p-\tilde{p}), F_{2} v_{n}-F_{2} p\right\rangle-v_{2}^{2}\left\|F_{2} v_{n}-F_{2} p\right\|^{2}
\end{aligned}
$$

and

$$
\begin{aligned}
\left\|k_{n}-p\right\|^{2} \leq & \left\|v_{n}-p\right\|^{2}-\left\|\left(\tilde{v}_{n}-k_{n}\right)+(p-\tilde{p})\right\|^{2} \\
& +2 v_{1}\left\|F_{1} \tilde{v}_{n}-F_{1} \tilde{p}\right\|\left\|\left(\tilde{v}_{n}-k_{n}\right)+(p-\tilde{p})\right\| .
\end{aligned}
$$

Consequently, from (3.4), (3.27), and (3.29), it follows that

$$
\begin{aligned}
\left\|y_{n}-p\right\|^{2} \leq & \beta_{n}\left\|x_{n}-p\right\|^{2}+\left(1-\beta_{n}\right)\left[\left\|\tilde{v}_{n}-\tilde{p}\right\|^{2}+v_{1}\left(v_{1}-2 \zeta_{1}\right)\left\|F_{1} \tilde{v}_{n}-F_{1} \tilde{p}\right\|^{2}\right] \\
\leq & \beta_{n}\left\|x_{n}-p\right\|^{2}+\left(1-\beta_{n}\right)\left\|\tilde{v}_{n}-\tilde{p}\right\|^{2} \\
\leq & \beta_{n}\left\|x_{n}-p\right\|^{2}+\left(1-\beta_{n}\right)\left[\left\|v_{n}-p\right\|^{2}-\left\|\left(v_{n}-\tilde{v}_{n}\right)-(p-\tilde{p})\right\|^{2}\right. \\
& \left.+2 v_{2}\left(\left(v_{n}-\tilde{v}_{n}\right)-(p-\tilde{p}), F_{2} v_{n}-F_{2} p\right\rangle-v_{2}^{2}\left\|F_{2} v_{n}-F_{2} p\right\|^{2}\right] \\
\leq & \beta_{n}\left\|x_{n}-p\right\|^{2}+\left(1-\beta_{n}\right)\left[\left\|x_{n}-p\right\|^{2}-\left\|\left(v_{n}-\tilde{v}_{n}\right)-(p-\tilde{p})\right\|^{2}\right. \\
& \left.+2 v_{2}\left\|\left(v_{n}-\tilde{v}_{n}\right)-(p-\tilde{p})\right\|\left\|F_{2} v_{n}-F_{2} p\right\|\right] \\
\leq & \left\|x_{n}-p\right\|^{2}-\left(1-\beta_{n}\right)\left\|\left(v_{n}-\tilde{v}_{n}\right)-(p-\tilde{p})\right\|^{2} \\
& +2 v_{2}\left\|\left(v_{n}-\tilde{v}_{n}\right)-(p-\tilde{p})\right\|\left\|F_{2} v_{n}-F_{2} p\right\|,
\end{aligned}
$$

which hence leads to

$$
\begin{aligned}
& \left(1-\beta_{n}\right)\left\|\left(v_{n}-\tilde{v}_{n}\right)-(p-\tilde{p})\right\|^{2} \\
& \quad \leq\left\|x_{n}-p\right\|^{2}-\left\|y_{n}-p\right\|^{2}+2 v_{2}\left\|\left(v_{n}-\tilde{v}_{n}\right)-(p-\tilde{p})\right\|\left\|F_{2} v_{n}-F_{2} p\right\| \\
& \quad \leq\left\|x_{n}-y_{n}\right\|\left(\left\|x_{n}-p\right\|+\left\|y_{n}-p\right\|\right)+2 v_{2}\left\|\left(v_{n}-\tilde{v}_{n}\right)-(p-\tilde{p})\right\|\left\|F_{2} v_{n}-F_{2} p\right\| .
\end{aligned}
$$


Since $\left\|x_{n}-y_{n}\right\| \rightarrow 0,\left\{\beta_{n}\right\} \subset[a, b] \subset(0,1), v_{2} \in\left(0,2 \zeta_{2}\right)$, and $\left\{x_{n}\right\},\left\{y_{n}\right\},\left\{v_{n}\right\},\left\{\tilde{v}_{n}\right\}$ are bounded sequences, we obtain from (3.28)

$$
\lim _{n \rightarrow \infty}\left\|\left(v_{n}-\tilde{v}_{n}\right)-(p-\tilde{p})\right\|=0
$$

Furthermore, from (3.4), (3.27), and (3.30), it follows that

$$
\begin{aligned}
\left\|y_{n}-p\right\|^{2} \leq & \beta_{n}\left\|x_{n}-p\right\|^{2}+\left(1-\beta_{n}\right)\left\|k_{n}-p\right\|^{2} \\
\leq & \beta_{n}\left\|x_{n}-p\right\|^{2}+\left(1-\beta_{n}\right)\left[\left\|v_{n}-p\right\|^{2}-\left\|\left(\tilde{v}_{n}-k_{n}\right)+(p-\tilde{p})\right\|^{2}\right. \\
& \left.+2 v_{1}\left\|F_{1} \tilde{v}_{n}-F_{1} \tilde{p}\right\|\left\|\left(\tilde{v}_{n}-k_{n}\right)+(p-\tilde{p})\right\|\right] \\
\leq & \beta_{n}\left\|x_{n}-p\right\|^{2}+\left(1-\beta_{n}\right)\left[\left\|x_{n}-p\right\|^{2}-\left\|\left(\tilde{v}_{n}-k_{n}\right)+(p-\tilde{p})\right\|^{2}\right. \\
& \left.+2 v_{1}\left\|F_{1} \tilde{v}_{n}-F_{1} \tilde{p}\right\|\left\|\left(\tilde{v}_{n}-k_{n}\right)+(p-\tilde{p})\right\|\right] \\
= & \left\|x_{n}-p\right\|^{2}-\left(1-\beta_{n}\right)\left\|\left(\tilde{v}_{n}-k_{n}\right)+(p-\tilde{p})\right\|^{2} \\
& +2 v_{1}\left\|F_{1} \tilde{v}_{n}-F_{1} \tilde{p}\right\|\left\|\left(\tilde{v}_{n}-k_{n}\right)+(p-\tilde{p})\right\|,
\end{aligned}
$$

which hence yields

$$
\begin{aligned}
& \left(1-\beta_{n}\right)\left\|\left(\tilde{v}_{n}-k_{n}\right)+(p-\tilde{p})\right\|^{2} \\
& \quad \leq\left\|x_{n}-p\right\|^{2}-\left\|y_{n}-p\right\|^{2}+2 v_{1}\left\|F_{1} \tilde{v}_{n}-F_{1} \tilde{p}\right\|\left\|\left(\tilde{v}_{n}-k_{n}\right)+(p-\tilde{p})\right\| \\
& \quad \leq\left\|x_{n}-y_{n}\right\|\left(\left\|x_{n}-p\right\|+\left\|y_{n}-p\right\|\right)+2 v_{1}\left\|F_{1} \tilde{v}_{n}-F_{1} \tilde{p}\right\|\left\|\left(\tilde{v}_{n}-k_{n}\right)+(p-\tilde{p})\right\| .
\end{aligned}
$$

Since $\left\|x_{n}-y_{n}\right\| \rightarrow 0,\left\{\beta_{n}\right\} \subset[a, b] \subset(0,1), v_{1} \in\left(0,2 \zeta_{1}\right)$, and $\left\{x_{n}\right\},\left\{y_{n}\right\},\left\{k_{n}\right\},\left\{\tilde{v}_{n}\right\}$ are bounded sequences, we obtain from (3.28)

$$
\lim _{n \rightarrow \infty}\left\|\left(\tilde{v}_{n}-k_{n}\right)+(p-\tilde{p})\right\|=0
$$

Note that

$$
\left\|v_{n}-k_{n}\right\| \leq\left\|\left(v_{n}-\tilde{v}_{n}\right)-(p-\tilde{p})\right\|+\left\|\left(\tilde{v}_{n}-k_{n}\right)+(p-\tilde{p})\right\| .
$$

Hence, from (3.31) and (3.32), we get

$$
\lim _{n \rightarrow \infty}\left\|v_{n}-G v_{n}\right\|=\lim _{n \rightarrow \infty}\left\|v_{n}-k_{n}\right\|=0
$$

Also, observe that

$$
y_{n}-x_{n}=\gamma_{n}\left(G v_{n}-x_{n}\right)+\delta_{n}\left(T G v_{n}-x_{n}\right), \quad \forall n \geq 0 \text {. }
$$

Hence, we obtain

$$
\begin{aligned}
\delta_{n}\left\|T G v_{n}-v_{n}\right\| & \leq \delta_{n}\left\|T G v_{n}-x_{n}\right\|+\delta_{n}\left\|x_{n}-v_{n}\right\| \\
& =\left\|y_{n}-x_{n}-\gamma_{n}\left(G v_{n}-x_{n}\right)\right\|+\delta_{n}\left\|x_{n}-v_{n}\right\| \\
& \leq\left\|y_{n}-x_{n}\right\|+\gamma_{n}\left\|G v_{n}-x_{n}\right\|+\delta_{n}\left\|x_{n}-v_{n}\right\|
\end{aligned}
$$




$$
\begin{aligned}
& \leq\left\|y_{n}-x_{n}\right\|+\gamma_{n}\left\|G v_{n}-v_{n}\right\|+\gamma_{n}\left\|v_{n}-x_{n}\right\|+\delta_{n}\left\|x_{n}-v_{n}\right\| \\
& =\left\|y_{n}-x_{n}\right\|+\gamma_{n}\left\|G v_{n}-v_{n}\right\|+\left(\gamma_{n}+\delta_{n}\right)\left\|x_{n}-v_{n}\right\| \\
& \leq\left\|y_{n}-x_{n}\right\|+\left\|G v_{n}-v_{n}\right\|+\left\|x_{n}-v_{n}\right\| .
\end{aligned}
$$

So, from $\liminf _{n \rightarrow \infty} \delta_{n}>0$, (3.16), (3.26), and (3.33), it follows that

$$
\lim _{n \rightarrow \infty}\left\|T G v_{n}-v_{n}\right\|=0 .
$$

In addition, noticing that

$$
\begin{aligned}
\left\|T v_{n}-v_{n}\right\| & \leq\left\|T v_{n}-T G v_{n}\right\|+\left\|T G v_{n}-v_{n}\right\| \\
& \leq\left\|v_{n}-G v_{n}\right\|+\left\|T G v_{n}-v_{n}\right\|,
\end{aligned}
$$

we know from (3.33) and (3.34) that

$$
\lim _{n \rightarrow \infty}\left\|T v_{n}-v_{n}\right\|=0
$$

Step 4. We prove that $\omega_{w}\left(x_{n}\right) \subset \Omega$.

Indeed, since $H$ is reflexive and $\left\{x_{n}\right\}$ is bounded, there exists at least a weak convergence subsequence of $\left\{x_{n}\right\}$. Hence it is well known that $\omega_{w}\left(x_{n}\right) \neq \emptyset$. Now, take an arbitrary $w \in$ $\omega_{w}\left(x_{n}\right)$. Then there exists a subsequence $\left\{x_{n_{i}}\right\}$ of $\left\{x_{n}\right\}$ such that $x_{n_{i}} \rightarrow w$. From (3.22)-(3.24) and (3.26) we have $u_{n_{i}} \rightarrow w, v_{n_{i}} \rightarrow w, \Lambda_{n_{i}}^{m} u_{n_{i}} \rightarrow w$, and $\Delta_{n_{i}}^{k} x_{n_{i}} \rightarrow w$, where $m \in\{1,2, \ldots, N\}$ and $k \in\{1,2, \ldots, M\}$. Utilizing Lemma 2.3(ii), we deduce from $v_{n_{i}} \rightarrow w$ and (3.35) that $w \in \operatorname{Fix}(T)$. In the meantime, utilizing Lemma 2.5, we obtain from $v_{n_{i}} \rightarrow w$ and (3.33) $w \in \operatorname{GSVI}(G)$. Next, we prove that $w \in \bigcap_{m=1}^{N} \mathrm{I}\left(B_{m}, R_{m}\right)$. As a matter of fact, since $B_{m}$ is $\eta_{m}$-inverse-strongly monotone, $B_{m}$ is a monotone and Lipschitz continuous mapping. It follows from Lemma 2.13 that $R_{m}+B_{m}$ is maximal monotone. Let $(v, g) \in G\left(R_{m}+B_{m}\right)$, that is, $g-B_{m} v \in R_{m} v$. Again, since $\Lambda_{n}^{m} u_{n}=J_{R_{m}, \lambda_{m, n}}\left(I-\lambda_{m, n} B_{m}\right) \Lambda_{n}^{m-1} u_{n}, n \geq 1, m \in\{1,2, \ldots, N\}$, we have

$$
\Lambda_{n}^{m-1} u_{n}-\lambda_{m, n} B_{m} \Lambda_{n}^{m-1} u_{n} \in\left(I+\lambda_{m, n} R_{m}\right) \Lambda_{n}^{m} u_{n}
$$

that is,

$$
\frac{1}{\lambda_{m, n}}\left(\Lambda_{n}^{m-1} u_{n}-\Lambda_{n}^{m} u_{n}-\lambda_{m, n} B_{m} \Lambda_{n}^{m-1} u_{n}\right) \in R_{m} \Lambda_{n}^{m} u_{n} .
$$

In terms of the monotonicity of $R_{m}$, we get

$$
\left\langle v-\Lambda_{n}^{m} u_{n}, g-B_{m} v-\frac{1}{\lambda_{m, n}}\left(\Lambda_{n}^{m-1} u_{n}-\Lambda_{n}^{m} u_{n}-\lambda_{m, n} B_{m} \Lambda_{n}^{m-1} u_{n}\right)\right\rangle \geq 0,
$$

and hence

$$
\begin{aligned}
& \left\langle v-\Lambda_{n}^{m} u_{n}, g\right\rangle \\
& \quad \geq\left\langle v-\Lambda_{n}^{m} u_{n}, B_{m} v+\frac{1}{\lambda_{m, n}}\left(\Lambda_{n}^{m-1} u_{n}-\Lambda_{n}^{m} u_{n}-\lambda_{m, n} B_{m} \Lambda_{n}^{m-1} u_{n}\right)\right\rangle
\end{aligned}
$$




$$
\begin{aligned}
& =\left\langle v-\Lambda_{n}^{m} u_{n}, B_{m} v-B_{m} \Lambda_{n}^{m} u_{n}+B_{m} \Lambda_{n}^{m} u_{n}-B_{m} \Lambda_{n}^{m-1} u_{n}+\frac{1}{\lambda_{m, n}}\left(\Lambda_{n}^{m-1} u_{n}-\Lambda_{n}^{m} u_{n}\right)\right\rangle \\
& \geq\left\langle v-\Lambda_{n}^{m} u_{n}, B_{m} \Lambda_{n}^{m} u_{n}-B_{m} \Lambda_{n}^{m-1} u_{n}\right\rangle+\left\langle v-\Lambda_{n}^{m} u_{n}, \frac{1}{\lambda_{m, n}}\left(\Lambda_{n}^{m-1} u_{n}-\Lambda_{n}^{m} u_{n}\right)\right\rangle .
\end{aligned}
$$

In particular,

$$
\begin{aligned}
\left\langle v-\Lambda_{n_{i}}^{m} u_{n_{i}}, g\right\rangle \geq & \left\langle v-\Lambda_{n_{i}}^{m} u_{n_{i}}, B_{m} \Lambda_{n_{i}}^{m} u_{n_{i}}-B_{m} \Lambda_{n_{i}}^{m-1} u_{n_{i}}\right\rangle \\
& +\left\langle v-\Lambda_{n_{i}}^{m} u_{n_{i}}, \frac{1}{\lambda_{m, n_{i}}}\left(\Lambda_{n_{i}}^{m-1} u_{n_{i}}-\Lambda_{n_{i}}^{m} u_{n_{i}}\right)\right\rangle .
\end{aligned}
$$

Since $\left\|\Lambda_{n}^{m} u_{n}-\Lambda_{n}^{m-1} u_{n}\right\| \rightarrow 0$ (due to (3.22)) and $\left\|B_{m} \Lambda_{n}^{m} u_{n}-B_{m} \Lambda_{n}^{m-1} u_{n}\right\| \rightarrow 0$ (due to the Lipschitz continuity of $\left.B_{m}\right)$, we conclude from $\Lambda_{n_{i}}^{m} u_{n_{i}} \rightarrow w$ and $\left\{\lambda_{i, n}\right\} \subset\left[a_{i}, b_{i}\right] \subset\left(0,2 \eta_{i}\right)$ that

$$
\lim _{i \rightarrow \infty}\left\langle v-\Lambda_{n_{i}}^{m} u_{n_{i}}, g\right\rangle=\langle v-w, g\rangle \geq 0
$$

It follows from the maximal monotonicity of $B_{m}+R_{m}$ that $0 \in\left(R_{m}+B_{m}\right) w$, that is, $w \in$ $\mathrm{I}\left(B_{m}, R_{m}\right)$. Therefore, $w \in \bigcap_{m=1}^{N} \mathrm{I}\left(B_{m}, R_{m}\right)$. Next we prove that $w \in \bigcap_{k=1}^{M} \operatorname{GMEP}\left(\Theta_{k}, \varphi_{k}, A_{k}\right)$. Since $\Delta_{n}^{k} x_{n}=T_{r_{k, n}}^{\left(\Theta_{k}, \varphi_{k}\right)}\left(I-r_{k, n} A_{k}\right) \Delta_{n}^{k-1} x_{n}, n \geq 1, k \in\{1,2, \ldots, M\}$, we have

$$
\begin{aligned}
& \Theta_{k}\left(\Delta_{n}^{k} x_{n}, y\right)+\varphi_{k}(y)-\varphi_{k}\left(\Delta_{n}^{k} x_{n}\right)+\left\langle A_{k} \Delta_{n}^{k-1} x_{n}, y-\Delta_{n}^{k} x_{n}\right\rangle \\
& +\frac{1}{r_{k, n}}\left\langle y-\Delta_{n}^{k} x_{n}, \Delta_{n}^{k} x_{n}-\Delta_{n}^{k-1} x_{n}\right\rangle \geq 0 .
\end{aligned}
$$

By (A2), we have

$$
\varphi_{k}(y)-\varphi_{k}\left(\Delta_{n}^{k} x_{n}\right)+\left\langle A_{k} \Delta_{n}^{k-1} x_{n}, y-\Delta_{n}^{k} x_{n}\right\rangle+\frac{1}{r_{k, n}}\left\langle y-\Delta_{n}^{k} x_{n}, \Delta_{n}^{k} x_{n}-\Delta_{n}^{k-1} x_{n}\right\rangle \geq \Theta_{k}\left(y, \Delta_{n}^{k} x_{n}\right) .
$$

Let $z_{t}=t y+(1-t) w$ for all $t \in(0,1]$ and $y \in C$. This implies that $z_{t} \in C$. Then we have

$$
\begin{aligned}
& \left\langle z_{t}-\Delta_{n}^{k} x_{n}, A_{k} z_{t}\right\rangle \\
& \geq \varphi_{k}\left(\Delta_{n}^{k} x_{n}\right)-\varphi_{k}\left(z_{t}\right)+\left\langle z_{t}-\Delta_{n}^{k} x_{n}, A_{k} z_{t}\right\rangle-\left\langle z_{t}-\Delta_{n}^{k} x_{n}, A_{k} \Delta_{n}^{k-1} x_{n}\right\rangle \\
& \quad-\left\langle z_{t}-\Delta_{n}^{k} x_{n}, \frac{\Delta_{n}^{k} x_{n}-\Delta_{n}^{k-1} x_{n}}{r_{k, n}}\right\rangle+\Theta_{k}\left(z_{t}, \Delta_{n}^{k} x_{n}\right) \\
& =\varphi_{k}\left(\Delta_{n}^{k} x_{n}\right)-\varphi_{k}\left(z_{t}\right)+\left\langle z_{t}-\Delta_{n}^{k} x_{n}, A_{k} z_{t}-A_{k} \Delta_{n}^{k} x_{n}\right\rangle \\
& \quad+\left\langle z_{t}-\Delta_{n}^{k} x_{n}, A_{k} \Delta_{n}^{k} x_{n}-A_{k} \Delta_{n}^{k-1} x_{n}\right\rangle-\left\langle z_{t}-\Delta_{n}^{k} x_{n}, \frac{\Delta_{n}^{k} x_{n}-\Delta_{n}^{k-1} x_{n}}{r_{k, n}}\right\rangle \\
& \quad+\Theta_{k}\left(z_{t}, \Delta_{n}^{k} x_{n}\right) .
\end{aligned}
$$

By (3.23), we have $\left\|A_{k} \Delta_{n}^{k} x_{n}-A_{k} \Delta_{n}^{k-1} x_{n}\right\| \rightarrow 0$ as $n \rightarrow \infty$. Furthermore, by the monotonicity of $A_{k}$, we obtain $\left\langle z_{t}-\Delta_{n}^{k} x_{n}, A_{k} z_{t}-A_{k} \Delta_{n}^{k} x_{n}\right\rangle \geq 0$. Then, by (A4), we obtain

$$
\left\langle z_{t}-w, A_{k} z_{t}\right\rangle \geq \varphi_{k}(w)-\varphi_{k}\left(z_{t}\right)+\Theta_{k}\left(z_{t}, w\right)
$$


Utilizing (A1), (A4), and (3.37), we have

$$
\begin{aligned}
0 & =\Theta_{k}\left(z_{t}, z_{t}\right)+\varphi_{k}\left(z_{t}\right)-\varphi_{k}\left(z_{t}\right) \\
& \leq t \Theta_{k}\left(z_{t}, y\right)+(1-t) \Theta_{k}\left(z_{t}, w\right)+t \varphi_{k}(y)+(1-t) \varphi_{k}(w)-\varphi_{k}\left(z_{t}\right) \\
& \leq t\left[\Theta_{k}\left(z_{t}, y\right)+\varphi_{k}(y)-\varphi_{k}\left(z_{t}\right)\right]+(1-t)\left\langle z_{t}-w, A_{k} z_{t}\right\rangle \\
& =t\left[\Theta_{k}\left(z_{t}, y\right)+\varphi_{k}(y)-\varphi_{k}\left(z_{t}\right)\right]+(1-t) t\left\langle y-w, A_{k} z_{t}\right\rangle
\end{aligned}
$$

and hence

$$
0 \leq \Theta_{k}\left(z_{t}, y\right)+\varphi_{k}(y)-\varphi_{k}\left(z_{t}\right)+(1-t)\left\langle y-w, A_{k} z_{t}\right\rangle
$$

Letting $t \rightarrow 0$, we have, for each $y \in C$,

$$
0 \leq \Theta_{k}(w, y)+\varphi_{k}(y)-\varphi_{k}(w)+\left\langle y-w, A_{k} w\right\rangle
$$

This implies that $w \in \operatorname{GMEP}\left(\Theta_{k}, \varphi_{k}, A_{k}\right)$, and hence, $w \in \bigcap_{k=1}^{M} \operatorname{GMEP}\left(\Theta_{k}, \varphi_{k}, A_{k}\right)$. Thus, $w \in \Omega=\bigcap_{n=1}^{\infty} \operatorname{Fix}\left(T_{n}\right) \cap \bigcap_{k=1}^{M} \operatorname{GMEP}\left(\Theta_{k}, \varphi_{k}, A_{k}\right) \cap \bigcap_{m=1}^{N} \mathrm{I}\left(B_{m}, R_{m}\right)$. Consequently, $w \in$ $\bigcap_{k=1}^{M} \operatorname{GMEP}\left(\Theta_{k}, \varphi_{k}, A_{k}\right) \cap \bigcap_{m=1}^{N} \mathrm{I}\left(B_{m}, R_{m}\right) \cap \operatorname{GSVI}(G) \cap \operatorname{Fix}(T)=: \Omega$. This shows that $\omega_{w}\left(x_{n}\right) \subset \Omega$.

Step 5. We prove that $\omega_{w}\left(x_{n}\right) \subset \Xi$.

Indeed, take an arbitrary $w \in \omega_{w}\left(x_{n}\right)$. Then there exists a subsequence $\left\{x_{n_{i}}\right\}$ of $\left\{x_{n}\right\}$ such that $x_{n_{i}} \rightarrow w$. Utilizing (3.15), we obtain for all $p \in \Omega$,

$$
\begin{aligned}
& \left\|x_{n+1}-p\right\|^{2} \\
& \leq\left(1-\lambda_{n} \frac{\tau^{2}-\gamma^{2}}{\tau}\right)\left\|x_{n}-p\right\|^{2}-\frac{\beta_{n}\left(1-\lambda_{n} \tau\right)}{1-\beta_{n}}\left\|y_{n}-x_{n}\right\|^{2} \\
& \quad+2 \lambda_{n} \alpha_{n}\left\langle(\gamma V p-\mu F p), x_{n+1}-p\right\rangle+2 \lambda_{n}\left(1-\alpha_{n}\right)\left\langle(\gamma S p-\mu F p), x_{n+1}-p\right\rangle \\
& \left.\left.\leq\left\|x_{n}-p\right\|^{2}+2 \lambda_{n} \alpha_{n}\right\}(\gamma V-\mu F) p, x_{n+1}-p\right\rangle+2 \lambda_{n}\left(1-\alpha_{n}\right)\left\langle(\gamma S p-\mu F p), x_{n+1}-p\right\rangle,
\end{aligned}
$$

which implies that

$$
\begin{aligned}
&\left\langle(\mu F-\gamma S) p, x_{n}-p\right\rangle \\
& \leq\left\langle(\mu F-\gamma S) p, x_{n}-x_{n+1}\right\rangle+\left\langle(\mu F-\gamma S) p, x_{n+1}-p\right\rangle \\
& \leq\|(\mu F-\gamma S) p\|\left\|x_{n}-x_{n+1}\right\|+\frac{\left\|x_{n}-p\right\|^{2}-\left\|x_{n+1}-p\right\|^{2}}{2 \lambda_{n}\left(1-\alpha_{n}\right)} \\
& \quad+\frac{\alpha_{n}}{1-\alpha_{n}}\left\langle(\gamma V-\mu F) p, x_{n+1}-p\right\rangle \\
& \leq\|(\mu F-\gamma S) p\|\left\|x_{n}-x_{n+1}\right\|+\frac{\left\|x_{n}-x_{n+1}\right\|\left(\left\|x_{n}-p\right\|+\left\|x_{n+1}-p\right\|\right)}{2 \lambda_{n}\left(1-\alpha_{n}\right)} \\
&+\frac{\alpha_{n}}{1-\alpha_{n}}\|(\gamma V-\mu F) p\|\left\|x_{n+1}-p\right\| .
\end{aligned}
$$

Since $\alpha_{n} \rightarrow 0,\left\|x_{n}-x_{n+1}\right\| \rightarrow 0$, and

$$
\lim _{n \rightarrow \infty} \frac{\left\|x_{n}-x_{n+1}\right\|}{\lambda_{n}}=\lim _{n \rightarrow \infty} \frac{\left\|x_{n}-x_{n+1}\right\|}{\alpha_{n}} \cdot \frac{\alpha_{n}}{\lambda_{n}}=0,
$$


from (3.38), we conclude that

$$
\begin{aligned}
\langle(\mu F-\gamma S) p, w-p\rangle & =\lim _{i \rightarrow \infty}\left\langle(\mu F-\gamma S) p, x_{n_{i}}-p\right\rangle \\
& \leq \limsup _{n \rightarrow \infty}\left\langle(\mu F-\gamma S) p, x_{n}-p\right\rangle \\
& \leq 0, \quad \forall p \in \Omega,
\end{aligned}
$$

that is,

$$
\langle(\mu F-\gamma S) p, w-p\rangle \leq 0, \quad \forall p \in \Omega
$$

In addition, observe that

$$
\begin{aligned}
\mu \eta \geq \tau & \Leftrightarrow \mu \eta \geq 1-\sqrt{1-\mu\left(2 \eta-\mu \kappa^{2}\right)} \\
& \Leftrightarrow \sqrt{1-\mu\left(2 \eta-\mu \kappa^{2}\right)} \geq 1-\mu \eta \\
& \Leftrightarrow 1-2 \mu \eta+\mu^{2} \kappa^{2} \geq 1-2 \mu \eta+\mu^{2} \eta^{2} \\
& \Leftrightarrow \kappa^{2} \geq \eta^{2} \\
& \Leftrightarrow \kappa \geq \eta
\end{aligned}
$$

and

$$
\begin{aligned}
\langle(\mu F-\gamma S) x-(\mu F-\gamma S) y, x-y\rangle & =\mu\langle F x-F y, x-y\rangle-\gamma\langle S x-S y, x-y\rangle \\
& \geq \mu \eta\|x-y\|^{2}-\gamma\|x-y\|^{2} \\
& =(\mu \eta-\gamma)\|x-y\|^{2}, \quad \forall x, y \in H .
\end{aligned}
$$

Since $0<\gamma \leq \tau$ and $\kappa \geq \eta$, we know that $\mu \eta \geq \tau \geq \gamma$ and hence the mapping $\mu F-\gamma S$ is monotone. Moreover, it is clear that the mapping $\mu F-\gamma S$ is $(\mu \kappa+\gamma)$-Lipschitzian. So, by Minty?s lemma B8], we know that (3.40) is equivalent to the VIP

$$
\langle(\mu F-\gamma S) w, p-w\rangle \geq 0, \quad \forall p \in \Omega
$$

This shows that $w \in \operatorname{VI}(\Omega, \mu F-\gamma S)=: \Xi$. Thus, we derive $\omega_{w}\left(x_{n}\right) \subset \Xi$ according to the arbitrariness of $w$.

Step 6. We prove that $\lim _{n \rightarrow \infty}\left\|x_{n}-x^{*}\right\|=0$ provided $\left\|y_{n}-T x_{n}\right\|=o\left(\alpha_{n}\right)$, where $\left\{x^{*}\right\}=$ $\mathrm{VI}(\Xi, \mu F-\gamma V)$.

Indeed, it is clear that $\mu F-\gamma V$ is $(\mu \eta-\gamma \rho)$-strongly monotone and $(\mu \kappa+\gamma \rho)$ Lipschitzian. Then it is well known that $\operatorname{VI}(\Xi, \mu F-\gamma V)$ is a singleton and hence we write $\operatorname{VI}(\Xi, \mu F-\gamma V)=\left\{x^{*}\right\}$, that is, $\operatorname{VI}(\operatorname{VI}(\Omega, \mu F-\gamma S), \mu F-\gamma V)=\left\{x^{*}\right\}$.

Utilizing (3.6) and (3.15) with $p=x^{*}$, we get

$$
\begin{aligned}
& \left\|x_{n+1}-x^{*}\right\|^{2} \\
& \quad \leq\left[\lambda_{n}\left(\alpha_{n} \gamma \rho\left\|x_{n}-x^{*}\right\|+\left(1-\alpha_{n}\right) \gamma\left\|x_{n}-x^{*}\right\|\right)+\left(1-\lambda_{n} \tau\right)\left\|y_{n}-x^{*}\right\|\right]^{2}
\end{aligned}
$$




$$
\begin{aligned}
& +2 \lambda_{n} \alpha_{n}\left|\left(\gamma V x^{*}-\mu F x^{*}\right), x_{n+1}-x^{*}\right\rangle+2\left(1-\alpha_{n}\right) \lambda_{n}\left\langle\left(\gamma S x^{*}-\mu F x^{*}\right), x_{n+1}-x^{*}\right\rangle \\
\leq & {\left[\lambda_{n} \gamma\left(1-\alpha_{n}(1-\rho)\right)\left\|x_{n}-x^{*}\right\|+\left(1-\lambda_{n} \tau\right)\left\|x_{n}-x^{*}\right\|\right]^{2} } \\
& +2 \lambda_{n} \alpha_{n}\left\langle\left(\gamma V x^{*}-\mu F x^{*}\right), x_{n+1}-x^{*}\right\rangle+2\left(1-\alpha_{n}\right) \lambda_{n}\left\langle\left(\gamma S x^{*}-\mu F x^{*}\right), x_{n+1}-x^{*}\right\rangle \\
= & {\left[\left(1-\lambda_{n}(\tau-\gamma)-\lambda_{n} \alpha_{n} \gamma(1-\rho)\right)\left\|x_{n}-x^{*}\right\|\right]^{2}+2 \lambda_{n} \alpha_{n}\left\langle(\gamma V-\mu F) x^{*}, x_{n+1}-x^{*}\right\rangle } \\
& +2\left(1-\alpha_{n}\right) \lambda_{n}\left((\gamma S-\mu F) x^{*}, x_{n+1}-x^{*}\right\rangle \\
\leq & \left(1-\lambda_{n}(\tau-\gamma)-\lambda_{n} \alpha_{n} \gamma(1-\rho)\right)\left\|x_{n}-x^{*}\right\|^{2}+2 \lambda_{n} \alpha_{n}\left|(\gamma V-\mu F) x^{*}, x_{n+1}-x^{*}\right\rangle \\
& +2\left(1-\alpha_{n}\right) \lambda_{n}\left(\left(\gamma S x^{*}-\mu F x^{*}\right), x_{n+1}-x^{*}\right\rangle \\
\leq & \left(1-\lambda_{n} \alpha_{n} \gamma(1-\rho)\right)\left\|x_{n}-x^{*}\right\|^{2}+2 \lambda_{n} \alpha_{n}\left|(\gamma V-\mu F) x^{*}, x_{n+1}-x^{*}\right\rangle \\
& +2\left(1-\alpha_{n}\right) \lambda_{n}\left((\gamma S-\mu F) x^{*}, x_{n+1}-x^{*}\right\rangle .
\end{aligned}
$$

Now let us show that

$$
\limsup _{n \rightarrow \infty}\left\langle(\gamma V-\mu F) x^{*}, x_{n}-x^{*}\right) \leq 0
$$

In fact, we may assume, without loss of generality, that there exists a subsequence $\left\{x_{n_{j}}\right\}$ of $\left\{x_{n}\right\}$ such that $x_{n_{j}} \rightarrow \hat{x}$ and

$$
\begin{aligned}
& \limsup _{n \rightarrow \infty}\left\langle(\gamma V-\mu F) x^{*}, x_{n}-x^{*}\right\rangle \\
& \quad=\lim _{j \rightarrow \infty}\left\langle(\gamma V-\mu F) x^{*}, x_{n_{j}}-x^{*}\right\rangle=\left\langle(\gamma V-\mu F) x^{*}, \hat{x}-x^{*}\right\rangle .
\end{aligned}
$$

In terms of the fact that $\omega_{w}\left(x_{n}\right) \subset \Xi$, we get $\hat{x} \in \Xi$. Since $\operatorname{VI}(\Xi, \mu F-\gamma V)=\left\{x^{*}\right\}$, it is easy to see from (3.45) that

$$
\limsup _{n \rightarrow \infty}\left\langle(\gamma V-\mu F) x^{*}, x_{n}-x^{*}\right\rangle=\left\langle(\gamma V-\mu F) x^{*}, \hat{x}-x^{*}\right\rangle \leq 0,
$$

that is, (3.44) holds.

In addition, from $x^{*} \in \Xi$ and condition (vi) we obtain

$$
\begin{aligned}
& \left\langle(\gamma S-\mu F) x^{*}, x_{n+1}-x^{*}\right\rangle \\
& \quad=\left\langle(\gamma S-\mu F) x^{*}, x_{n+1}-P_{\Omega} x_{n+1}\right\rangle+\left\langle(\gamma S-\mu F) x^{*}, P_{\Omega} x_{n+1}-x^{*}\right\rangle \\
& \quad \leq\left\langle(\gamma S-\mu F) x^{*}, x_{n+1}-P_{\Omega} x_{n+1}\right\rangle \\
& \leq\left\|(\gamma S-\mu F) x^{*}\right\| d\left(x_{n+1}, \Omega\right) \\
& \quad \leq\left\|(\gamma S-\mu F) x^{*}\right\|\left(\frac{1}{\bar{k}}\left\|x_{n+1}-T x_{n+1}\right\|\right)^{1 / \theta} .
\end{aligned}
$$

Utilizing Lemma 2.3(i) we have

$$
\begin{aligned}
& \left\|x_{n+1}-T x_{n+1}\right\| \\
& \quad \leq\left\|x_{n+1}-T x_{n}\right\|+\left\|T x_{n}-T x_{n+1}\right\|
\end{aligned}
$$




$$
\begin{aligned}
& \leq \frac{1+\xi}{1-\xi}\left\|x_{n}-x_{n+1}\right\|+\left\|x_{n+1}-T x_{n}\right\| \\
& =\frac{1+\xi}{1-\xi}\left\|x_{n}-x_{n+1}\right\|+\left\|\lambda_{n} \gamma\left(\alpha_{n} V x_{n}+\left(1-\alpha_{n}\right) S x_{n}\right)+\left(I-\lambda_{n} \mu F\right) y_{n}-T x_{n}\right\| \\
& \leq \frac{1+\xi}{1-\xi}\left\|x_{n}-x_{n+1}\right\|+\left\|y_{n}-T x_{n}\right\|+\lambda_{n}\left\|\gamma\left(\alpha_{n} V x_{n}+\left(1-\alpha_{n}\right) S x_{n}\right)-\mu F y_{n}\right\| \\
& \leq \frac{1+\xi}{1-\xi}\left\|x_{n}-x_{n+1}\right\|+\left\|y_{n}-T x_{n}\right\|+\lambda_{n} \tilde{M}_{5},
\end{aligned}
$$

where $\sup _{n \geq 0}\left\|\gamma \alpha_{n}\left(V x_{n}-S x_{n}\right)+\gamma S x_{n}-\mu F y_{n}\right\| \leq \widetilde{M}_{5}$ for some $\widetilde{M}_{5}>0$. Hence, for a big enough constant $\bar{k}_{1}>0$, from (3.46), we have

$$
\begin{aligned}
& \left\langle(\gamma S-\mu F) x^{*}, x_{n+1}-x^{*}\right\rangle \\
& \leq\left\|(\gamma S-\mu F) x^{*}\right\|\left(\frac{1}{\bar{k}}\left\|x_{n+1}-T x_{n+1}\right\|\right)^{1 / \theta} \\
& \leq\left\|(\gamma S-\mu F) x^{*}\right\|\left[\frac{1}{\bar{k}}\left(\frac{1+\xi}{1-\xi}\left\|x_{n}-x_{n+1}\right\|+\left\|y_{n}-T x_{n}\right\|+\lambda_{n} \widetilde{M}_{5}\right)\right]^{1 / \theta} \\
& \leq \bar{k}_{1}\left(\lambda_{n}+\left\|x_{n}-x_{n+1}\right\|+\left\|y_{n}-T x_{n}\right\|\right)^{1 / \theta} \\
& \quad \bar{k}_{1} \lambda_{n}^{1 / \theta}\left(1+\frac{\left\|x_{n}-x_{n+1}\right\|+\left\|y_{n}-T x_{n}\right\|}{\lambda_{n}}\right)^{1 / \theta} .
\end{aligned}
$$

Combining (3.43) and (3.48), we get

$$
\begin{aligned}
\| x_{n+1} & -x^{*} \|^{2} \\
\leq & \left(1-\lambda_{n} \alpha_{n} \gamma(1-\rho)\right)\left\|x_{n}-x^{*}\right\|^{2}+2 \lambda_{n} \alpha_{n}\left\langle(\gamma V-\mu F) x^{*}, x_{n+1}-x^{*}\right\rangle \\
& +2\left(1-\alpha_{n}\right) \lambda_{n}\left\langle(\gamma S-\mu F) x^{*}, x_{n+1}-x^{*}\right\rangle \\
= & \left(1-\lambda_{n} \alpha_{n} \gamma(1-\rho)\right)\left\|x_{n}-x^{*}\right\|^{2}+\lambda_{n} \alpha_{n} \gamma(1-\rho) \cdot \frac{2}{\gamma(1-\rho)}\left[\left\langle(\gamma V-\mu F) x^{*}, x_{n+1}-x^{*}\right\rangle\right. \\
& \left.+\frac{\left(1-\alpha_{n}\right)\left\langle(\gamma S-\mu F) x^{*}, x_{n+1}-x^{*}\right\rangle}{\alpha_{n}}\right] \\
\leq & \left(1-\lambda_{n} \alpha_{n} \gamma(1-\rho)\right)\left\|x_{n}-x^{*}\right\|^{2}+\lambda_{n} \alpha_{n} \gamma(1-\rho) \cdot \frac{2}{\gamma(1-\rho)}\left[\left\langle(\gamma V-\mu F) x^{*}, x_{n+1}-x^{*}\right\rangle\right. \\
& \left.+\bar{k}_{1} \frac{\lambda_{n}^{1 / \theta}}{\alpha_{n}}\left(1+\frac{\left\|x_{n}-x_{n+1}\right\|+\left\|y_{n}-T x_{n}\right\|}{\lambda_{n}}\right)^{1 / \theta}\right] .
\end{aligned}
$$

Since $\sum_{n=0}^{\infty} \lambda_{n} \alpha_{n}=\infty, \limsup _{n \rightarrow \infty} \frac{\alpha_{n}}{\lambda_{n}}<\infty,\left\|x_{n}-x_{n+1}\right\|=o\left(\alpha_{n}\right)$, and $\frac{\lambda_{n}^{1 / \theta}}{\alpha_{n}} \rightarrow 0$, we conclude from (3.44) and the assumption $\left\|y_{n}-T x_{n}\right\|=o\left(\alpha_{n}\right)$ that $\sum_{n=0}^{\infty} \lambda_{n} \alpha_{n} \gamma(1-\rho)=\infty$,

$$
\lim _{n \rightarrow \infty} \frac{\left\|x_{n}-x_{n+1}\right\|+\left\|y_{n}-T x_{n}\right\|}{\lambda_{n}}=\lim _{n \rightarrow \infty}\left(\frac{\left\|x_{n}-x_{n+1}\right\|}{\alpha_{n}}+\frac{\left\|y_{n}-T x_{n}\right\|}{\alpha_{n}}\right) \frac{\alpha_{n}}{\lambda_{n}}=0
$$

and

$$
\lim _{n \rightarrow \infty} \frac{2}{\gamma(1-\rho)}\left[\left\langle(\gamma V-\mu F) x^{*}, x_{n+1}-x^{*}\right\rangle+\bar{k}_{1} \frac{\lambda_{n}^{1 / \theta}}{\alpha_{n}}\left(1+\frac{\left\|x_{n}-x_{n+1}\right\|+\left\|y_{n}-T x_{n}\right\|}{\lambda_{n}}\right)^{1 / \theta}\right] \leq 0 .
$$


Therefore, applying Lemma 2.8 to (3.49) we infer that $\lim _{n \rightarrow \infty}\left\|x_{n}-x^{*}\right\|=0$. The proof of part (a) is complete.

It is easy to see that part (b) now becomes a straightforward consequence of part (a) since, if $V \equiv 0$, THVIP (1.10) reduces to the VIP in part (b). This completes the proof.

Remark 3.1 It is clear that the iterative scheme (3.1) is different from the one considered in $[7,32]$. We extend the three-step iterative scheme in [7, Algorithm I] to the four-step iterative scheme for THVIP (1.10) by combining Korpelevich?s extragradient method, the viscosity approximation method, the hybrid steepest-descent method [41] and Mann?s iteration method. It is worth pointing out that under the lack of assumptions similar to those in [32, Theorem 3.2], for example, $\left\{x_{n}\right\}$ is bounded and Fix $(T) \cap \operatorname{int} C \neq \emptyset$, the sequence $\left\{x_{n}\right\}$ generated by (3.1) converges strongly to a point $x^{*} \in \bigcap_{k=1}^{M} \operatorname{GMEP}\left(\Theta_{k}, \varphi_{k}, A_{k}\right) \cap$ $\bigcap_{i=1}^{N} \mathrm{I}\left(B_{i}, R_{i}\right) \cap \operatorname{GSVI}(G) \cap \operatorname{Fix}(T)=: \Omega$, which is a unique solution $x^{*} \in \Xi$ of THVIP (1.10) (over the fixed point set of a strictly pseudocontractive mapping $T$ ), that is, $\langle(\mu F-$ $\left.\gamma V) x^{*}, p-x^{*}\right\rangle \geq 0, \forall p \in \Xi$. We note that the nonexpansive mapping $T$ in [32] is extended to a strictly pseudocontractive mapping $T$ in (3.1).

Remark 3.2 Theorem 3.1 improves and extends Theorems 3.1 and 3.2 in [32] and Theorem 14 in [7] in the following aspects:

(a) THVIP (1.10) with the unique solution $x^{*} \in \Omega$ satisfying

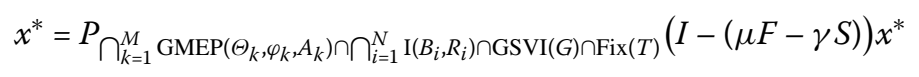

is more general than the problem of finding a point $\tilde{x} \in C$ satisfying $\tilde{x}=P_{\operatorname{Fix}(T)} S \tilde{x}$ in [32] and than the problem of finding a point $x^{*} \in \operatorname{Fix}(T) \cap \operatorname{VI}(C, A)$ satisfying $x^{*}=P_{\mathrm{Fix}(T) \cap \mathrm{VI}(C, A)}(I-(\mu F-\gamma S)) x^{*}$ in [7, Theorem 14]. It is worth to point out that $S$ is nonexpansive if and only if the complement $I-S$ is $\frac{1}{2}$-inverse-strongly monotone; see [15].

(b) The four-step iterative scheme (3.1) for THVIP (1.10) is more flexible and subtle than the three-step iterative scheme considered in [7, Algorithm I] and than the two-step iterative scheme studied in [32] because it can be used to solve several kinds of problems, for example, THVIP, HVIP, and the problem of finding a common point of four sets: $\bigcap_{k=1}^{M} \operatorname{GMEP}\left(\Theta_{k}, \varphi_{k}, A_{k}\right), \bigcap_{i=1}^{N} \mathrm{I}\left(B_{i}, R_{i}\right), \operatorname{GSVI}(G)$, and $\operatorname{Fix}(T)$. In addition, Theorem 3.1 drops the crucial requirements in [32, Theorem 3.2] that $\lim _{n \rightarrow \infty} \frac{\alpha_{n}}{\beta_{n}}=0, \lim _{n \rightarrow \infty} \frac{\beta_{n}^{2}}{\alpha_{n}}=0, \operatorname{Fix}(T) \cap \operatorname{int} C \neq \emptyset$, and $\left\{x_{n}\right\}$ is bounded, and also removes the crucial ones in [7, Theorem 14] that $\sum_{n=0}^{\infty} \alpha_{n}<\infty, \lim _{n \rightarrow \infty}\left(\alpha_{n} / \lambda_{n}^{2}\right)=0$, and $\left\|x_{n+1}-x_{n}\right\|+\left\|x_{n}-z_{n}\right\|=o\left(\lambda_{n}^{2}\right)$. In the meantime, Problem 1.1 (that is, Problem II in [7]) is extended and generalized to the setting of GSVI (1.4), finitely many GMEPs, and finitely many variational inclusions in Problem 1.2.

(c) The technique used in Theorem 3.1 is different from the one used in [32, Theorems 3.1 and 3.2] and in [7, Theorem 14] because we used the properties of strictly pseudocontractive mappings (see Lemmas 2.3 and 2.4), the properties of resolvent operators and maximal monotone mappings (see Proposition 2.2, Remark 2.2 and Lemmas 2.9-2.13), the fixed point equation $x^{*}=P_{C}\left(I-v_{1} F_{1}\right) P_{C}\left(I-v_{2} F_{2}\right) x^{*}$ equivalent to GSVI (1.4) (see Proposition 2.3), and contractive coefficient estimates for contractions associated with nonexpansive mappings (see Lemma 2.7). 
(d) Compared with the restrictions on the parameter sequences in [32, Theorem 3.2] and [7, Theorem 14], respectively, the hypotheses (iii)-(iv) in Theorem 3.1 are additionally added because Theorem 3.1 involves a quite complex problem, that is, THVIP (1.10) (over the fixed point set Fix $(T)$ of a strictly pseudocontractive mapping $T$ ) with constraints of several problems: GSVI (1.4), finitely many GMEPs, and finitely many variational inclusions.

We prove the strong convergence of the proposed algorithm to a unique solution of THVI Problem 1.2.

Theorem 3.2 In addition to Assumption 3.1, suppose that

(i) $\lim _{n \rightarrow \infty} \lambda_{n}=0, \lim _{n \rightarrow \infty} \alpha_{n}=0$ and $\sum_{n=0}^{\infty} \lambda_{n} \alpha_{n}=\infty$;

(ii) $\beta_{n}+\gamma_{n}+\delta_{n}=1$ and $\left(\gamma_{n}+\delta_{n}\right) \xi \leq \gamma_{n}$ for all $n \geq 0$;

(iii) $\liminf _{n \rightarrow \infty} \delta_{n}>0$ and $\left\{\beta_{n}\right\} \subset[a, b] \subset(0,1)$;

(iv) $\lim _{n \rightarrow \infty}\left(\lambda_{n} / \alpha_{n}^{2}\right)=0$ and $\|x-T x\| \geq \bar{k} \cdot d(x, \Omega), \forall x \in C$, for some $\bar{k}$.

If $\left\{S x_{n}\right\}$ is bounded, then

(a) $\omega_{w}\left(x_{n}\right) \subset \Omega$ provided $\left\|x_{n}-x_{n+1}\right\| \rightarrow 0(n \rightarrow \infty)$;

(b) $\omega_{w}\left(x_{n}\right) \subset \Xi$ provided $\left\|x_{n}-x_{n+1}\right\|=o\left(\lambda_{n}\right)$;

(c) $\left\{x_{n}\right\}$ converges strongly to a unique solution of Problem 1.2 provided $\left\|x_{n}-x_{n+1}\right\|+\left\|x_{n}-T x_{n}\right\|=o\left(\lambda_{n} \alpha_{n}^{2}\right)$

Proof Since the solution set $\Xi$ of the HVIP (1.11) is nonempty, it is well known that $\Omega \neq \emptyset$. As in the proof of Theorem 3.1, put

$$
\Delta_{n}^{k}=T_{r_{k, n}}^{\left(\Theta_{k}, \varphi_{k}\right)}\left(I-r_{k, n} A_{k}\right) T_{r_{k-1, n}}^{\left(\Theta_{k-1}, \varphi_{k-1}\right)}\left(I-r_{k-1, n} A_{k-1}\right) \cdots T_{r_{1, n}}^{\left(\Theta_{1}, \varphi_{1}\right)}\left(I-r_{1, n} A_{1}\right) x_{n}
$$

for all $k \in\{1,2, \ldots, M\}$ and $n \geq 1$,

$$
\Lambda_{n}^{i}=J_{R_{i}, \lambda_{i, n}}\left(I-\lambda_{i, n} B_{i}\right) J_{R_{i-1}, \lambda_{i-1, n}}\left(I-\lambda_{i-1, n} B_{i-1}\right) \cdots J_{R_{1}, \lambda_{1, n}}\left(I-\lambda_{1, n} B_{1}\right)
$$

for all $i \in\{1,2, \ldots, N\}, \Delta_{n}^{0}=I$, and $\Lambda_{n}^{0}=I$, where $I$ is the identity mapping on $H$. Then we have $u_{n}=\Delta_{n}^{M} x_{n}$ and $v_{n}=\Lambda_{n}^{N} u_{n}$.

The rest of the proof is divided into several steps.

Step 1. As in the proof (Step 1) of Theorem 3.1, $\left\{x_{n}\right\}$ is bounded.

Step 2. We prove that $\omega_{w}\left(x_{n}\right) \subset \Omega$ provided $\left\|x_{n}-x_{n+1}\right\| \rightarrow 0(n \rightarrow \infty)$.

Indeed, we first show that $\lim _{n \rightarrow \infty}\left\|x_{n}-u_{n}\right\|=0, \lim _{n \rightarrow \infty}\left\|x_{n}-v_{n}\right\|=0, \lim _{n \rightarrow \infty} \| v_{n}-$ $G v_{n} \|=0$, and $\lim _{n \rightarrow \infty}\left\|v_{n}-T v_{n}\right\|=0$.

As a matter of fact, utilizing Lemmas 2.1 and 2.2(b), from (3.1), (3.4), (3.5), and $0<\gamma \leq \tau$, we deduce

$$
\begin{aligned}
& \left\|y_{n}-p\right\|^{2} \\
& =\left\|\beta_{n} x_{n}+\gamma_{n} G v_{n}+\delta_{n} T G v_{n}-p\right\|^{2} \\
& =\left\|\beta_{n}\left(x_{n}-p\right)+\left(1-\beta_{n}\right)\left(\frac{\gamma_{n} G v_{n}+\delta_{n} T G v_{n}}{1-\beta_{n}}-p\right)\right\|^{2} \\
& =\beta_{n}\left\|x_{n}-p\right\|^{2}+\left(1-\beta_{n}\right)\left\|\frac{\gamma_{n} G v_{n}+\delta_{n} T G v_{n}}{1-\beta_{n}}-p\right\|^{2}
\end{aligned}
$$




$$
\begin{aligned}
& -\beta_{n}\left(1-\beta_{n}\right)\left\|\frac{\gamma_{n} G v_{n}+\delta_{n} T G v_{n}}{1-\beta_{n}}-x_{n}\right\|^{2} \\
= & \beta_{n}\left\|x_{n}-p\right\|^{2}+\left(1-\beta_{n}\right)\left\|\frac{\gamma_{n}\left(G v_{n}-p\right)+\delta_{n}\left(T G v_{n}-p\right)}{1-\beta_{n}}\right\|^{2}-\beta_{n}\left(1-\beta_{n}\right)\left\|\frac{y_{n}-x_{n}}{1-\beta_{n}}\right\|^{2} \\
\leq & \beta_{n}\left\|x_{n}-p\right\|^{2}+\left(1-\beta_{n}\right) \frac{\left(\gamma_{n}+\delta_{n}\right)^{2}\left\|G v_{n}-p\right\|^{2}}{\left(1-\beta_{n}\right)^{2}}-\frac{\beta_{n}}{1-\beta_{n}}\left\|y_{n}-x_{n}\right\|^{2} \\
= & \beta_{n}\left\|x_{n}-p\right\|^{2}+\left(1-\beta_{n}\right)\left\|G v_{n}-p\right\|^{2}-\frac{\beta_{n}}{1-\beta_{n}}\left\|y_{n}-x_{n}\right\|^{2} \\
\leq & \beta_{n}\left\|x_{n}-p\right\|^{2}+\left(1-\beta_{n}\right)\left\|v_{n}-p\right\|^{2}-\frac{\beta_{n}}{1-\beta_{n}}\left\|y_{n}-x_{n}\right\|^{2} \\
\leq & \beta_{n}\left\|x_{n}-p\right\|^{2}+\left(1-\beta_{n}\right)\left\|x_{n}-p\right\|^{2}-\frac{\beta_{n}}{1-\beta_{n}}\left\|y_{n}-x_{n}\right\|^{2} \\
= & \left\|x_{n}-p\right\|^{2}-\frac{\beta_{n}}{1-\beta_{n}}\left\|y_{n}-x_{n}\right\|^{2},
\end{aligned}
$$

and hence

$$
\begin{aligned}
\left\|x_{n+1}-p\right\|^{2} & \left\|\lambda_{n} \gamma\left(\alpha_{n} V x_{n}+\left(1-\alpha_{n}\right) S x_{n}\right)+\left(I-\lambda_{n} \mu F\right) y_{n}-p\right\|^{2} \\
= & \left\|\lambda_{n} \gamma\left(\alpha_{n} V x_{n}+\left(1-\alpha_{n}\right) S x_{n}\right)-\lambda_{n} \mu F p+\left(I-\lambda_{n} \mu F\right) y_{n}-\left(I-\lambda_{n} \mu F\right) p\right\|^{2} \\
= & \left\|\lambda_{n}\left[\alpha_{n}\left(\gamma V x_{n}-\mu F p\right)+\left(1-\alpha_{n}\right)\left(\gamma S x_{n}-\mu F p\right)\right]+\left(I-\lambda_{n} \mu F\right) y_{n}-\left(I-\lambda_{n} \mu F\right) p\right\|^{2} \\
= & \| \lambda_{n}\left[\alpha_{n}\left(\gamma V x_{n}-\gamma V p\right)+\left(1-\alpha_{n}\right)\left(\gamma S x_{n}-\gamma S p\right)\right]+\left(I-\lambda_{n} \mu F\right) y_{n}-\left(I-\lambda_{n} \mu F\right) p \\
& +\lambda_{n}\left[\alpha_{n}(\gamma V p-\mu F p)+\left(1-\alpha_{n}\right)(\gamma S p-\mu F p)\right] \|^{2} \\
\leq & \left\|\lambda_{n}\left[\alpha_{n}\left(\gamma V x_{n}-\gamma V p\right)+\left(1-\alpha_{n}\right)\left(\gamma S x_{n}-\gamma S p\right)\right]+\left(I-\lambda_{n} \mu F\right) y_{n}-\left(I-\lambda_{n} \mu F\right) p\right\|^{2} \\
& +2 \lambda_{n} \alpha_{n}\left|(\gamma V p-\mu F p), x_{n+1}-p\right\rangle+2 \lambda_{n}\left(1-\alpha_{n}\right)\left|(\gamma S p-\mu F p), x_{n+1}-p\right\rangle \\
\leq & {\left[\lambda_{n}\left\|\alpha_{n}\left(\gamma V x_{n}-\gamma V p\right)+\left(1-\alpha_{n}\right)\left(\gamma S x_{n}-\gamma S p\right)\right\|+\left\|\left(I-\lambda_{n} \mu F\right) y_{n}-\left(I-\lambda_{n} \mu F\right) p\right\|\right]^{2} } \\
& +2 \lambda_{n} \alpha_{n}\left|(\gamma V p-\mu F p), x_{n+1}-p\right\rangle+2 \lambda_{n}\left(1-\alpha_{n}\right)\left\langle(\gamma S p-\mu F p), x_{n+1}-p\right\rangle \\
\leq & {\left[\lambda_{n}\left(\alpha_{n} \gamma \rho\left\|x_{n}-p\right\|+\left(1-\alpha_{n}\right) \gamma\left\|x_{n}-p\right\|\right)+\left(1-\lambda_{n} \tau\right)\left\|y_{n}-p\right\|\right]^{2} } \\
& +2 \lambda_{n} \alpha_{n}\left|(\gamma V p-\mu F p), x_{n+1}-p\right\rangle+2\left(1-\alpha_{n}\right) \lambda_{n}\left|(\gamma S p-\mu F p), x_{n+1}-p\right\rangle \\
= & {\left[\lambda_{n}\left(1-\alpha_{n}(1-\rho)\right) \gamma\left\|x_{n}-p\right\|+\left(1-\lambda_{n} \tau\right)\left\|y_{n}-p\right\|\right]^{2} } \\
& +2 \lambda_{n} \alpha_{n}\left|(\gamma V p-\mu F p), x_{n+1}-p\right\rangle+2 \lambda_{n}\left(1-\alpha_{n}\right)\left|(\gamma S p-\mu F p), x_{n+1}-p\right\rangle \\
\leq & {\left[\lambda_{n} \gamma\left\|x_{n}-p\right\|+\left(1-\lambda_{n} \tau\right)\left\|y_{n}-p\right\|\right]^{2} } \\
& +2 \lambda_{n} \alpha_{n}\left|(\gamma V p-\mu F p), x_{n+1}-p\right\rangle+2 \lambda_{n}\left(1-\alpha_{n}\right)\left((\gamma S p-\mu F p), x_{n+1}-p\right\rangle \\
= & {\left[\lambda_{n} \tau \cdot \frac{\gamma}{\tau}\left\|x_{n}-p\right\|+\left(1-\lambda_{n} \tau\right)\left\|y_{n}-p\right\|\right]^{2} } \\
& +2 \lambda_{n} \alpha_{n}\left|(\gamma V p-\mu F p), x_{n+1}-p\right\rangle+2 \lambda_{n}\left(1-\alpha_{n}\right)\left\langle(\gamma S p-\mu F p), x_{n+1}-p\right\rangle \\
\leq & \lambda_{n} \frac{\gamma^{2}}{\tau}\left\|x_{n}-p\right\|^{2}+\left(1-\lambda_{n} \tau\right)\left\|y_{n}-p\right\|^{2} \\
& +2 \lambda_{n} \alpha_{n}\left|(\gamma V p-\mu F p), x_{n+1}-p\right\rangle+2 \lambda_{n}\left(1-\alpha_{n}\right)\left|(\gamma S p-\mu F p), x_{n+1}-p\right\rangle \\
= &
\end{aligned}
$$




$$
\begin{aligned}
\leq & \lambda_{n} \frac{\gamma^{2}}{\tau}\left\|x_{n}-p\right\|^{2}+\left(1-\lambda_{n} \tau\right)\left[\left\|x_{n}-p\right\|^{2}-\frac{\beta_{n}}{1-\beta_{n}}\left\|y_{n}-x_{n}\right\|^{2}\right] \\
& +2 \lambda_{n} \alpha_{n}\left\langle(\gamma V p-\mu F p), x_{n+1}-p\right\rangle+2 \lambda_{n}\left(1-\alpha_{n}\right)\left\langle(\gamma S p-\mu F p), x_{n+1}-p\right\rangle \\
= & \left(1-\lambda_{n} \frac{\tau^{2}-\gamma^{2}}{\tau}\right)\left\|x_{n}-p\right\|^{2}-\frac{\beta_{n}\left(1-\lambda_{n} \tau\right)}{1-\beta_{n}}\left\|y_{n}-x_{n}\right\|^{2} \\
& +2 \lambda_{n} \alpha_{n}\left\langle(\gamma V p-\mu F p), x_{n+1}-p\right\rangle+2 \lambda_{n}\left(1-\alpha_{n}\right)\left\langle(\gamma S p-\mu F p), x_{n+1}-p\right\rangle \\
\leq & \left\|x_{n}-p\right\|^{2}-\frac{\beta_{n}\left(1-\lambda_{n} \tau\right)}{1-\beta_{n}}\left\|y_{n}-x_{n}\right\|^{2} \\
& +2 \lambda_{n} \alpha_{n}\|\gamma V p-\mu F p\|\left\|x_{n+1}-p\right\|+2 \lambda_{n}\|\gamma S p-\mu F p\|\left\|x_{n+1}-p\right\|,
\end{aligned}
$$

which together with $\left\{\beta_{n}\right\} \subset[a, b] \subset(0,1)$ yields

$$
\begin{aligned}
& \frac{a\left(1-\lambda_{n} \tau\right)}{1-a}\left\|y_{n}-x_{n}\right\|^{2} \\
& \leq \frac{\beta_{n}\left(1-\lambda_{n} \tau\right)}{1-\beta_{n}}\left\|y_{n}-x_{n}\right\|^{2} \\
& \leq\left\|x_{n}-p\right\|^{2}-\left\|x_{n+1}-p\right\|^{2}+2 \lambda_{n} \alpha_{n}\|\gamma V p-\mu F p\|\left\|x_{n+1}-p\right\| \\
& \quad+2 \lambda_{n}\|\gamma S p-\mu F p\|\left\|x_{n+1}-p\right\| \\
& \leq\left\|x_{n}-x_{n+1}\right\|\left(\left\|x_{n}-p\right\|+\left\|x_{n+1}-p\right\|\right)+2 \lambda_{n} \alpha_{n}\|\gamma V p-\mu F p\|\left\|x_{n+1}-p\right\| \\
& \quad+2 \lambda_{n}\|\gamma S p-\mu F p\|\left\|x_{n+1}-p\right\| .
\end{aligned}
$$

Since $\alpha_{n} \rightarrow 0, \lambda_{n} \rightarrow 0,\left\|x_{n+1}-x_{n}\right\| \rightarrow 0$, and $\left\{x_{n}\right\}$ is bounded, we have

$$
\lim _{n \rightarrow \infty}\left\|y_{n}-x_{n}\right\|=0
$$

Observe that

$$
\begin{aligned}
\left\|\Delta_{n}^{k} x_{n}-p\right\|^{2} & =\left\|T_{r_{k, n}}^{\left(\Theta_{k}, \varphi_{k}\right)}\left(I-r_{k, n} A_{k}\right) \Delta_{n}^{k-1} x_{n}-T_{r_{k, n}}^{\left(\Theta_{k}, \varphi_{k}\right)}\left(I-r_{k, n} A_{k}\right) p\right\|^{2} \\
& \leq\left\|\left(I-r_{k, n} A_{k}\right) \Delta_{n}^{k-1} x_{n}-\left(I-r_{k, n} A_{k}\right) p\right\|^{2} \\
& \leq\left\|\Delta_{n}^{k-1} x_{n}-p\right\|^{2}+r_{k, n}\left(r_{k, n}-2 \mu_{k}\right)\left\|A_{k} \Delta_{n}^{k-1} x_{n}-A_{k} p\right\|^{2} \\
& \leq\left\|x_{n}-p\right\|^{2}+r_{k, n}\left(r_{k, n}-2 \mu_{k}\right)\left\|A_{k} \Delta_{n}^{k-1} x_{n}-A_{k} p\right\|^{2}
\end{aligned}
$$

and

$$
\begin{aligned}
\left\|\Lambda_{n}^{i} u_{n}-p\right\|^{2} & =\left\|J_{R_{i}, \lambda_{i, n}}\left(I-\lambda_{i, n} B_{i}\right) \Lambda_{n}^{i-1} u_{n}-J_{R_{i}, \lambda_{i, n}}\left(I-\lambda_{i, n} B_{i}\right) p\right\|^{2} \\
& \leq\left\|\left(I-\lambda_{i, n} B_{i}\right) \Lambda_{n}^{i-1} u_{n}-\left(I-\lambda_{i, n} B_{i}\right) p\right\|^{2} \\
& \leq\left\|\Lambda_{n}^{i-1} u_{n}-p\right\|^{2}+\lambda_{i, n}\left(\lambda_{i, n}-2 \eta_{i}\right)\left\|B_{i} \Lambda_{n}^{i-1} u_{n}-B_{i} p\right\|^{2} \\
& \leq\left\|u_{n}-p\right\|^{2}+\lambda_{i, n}\left(\lambda_{i, n}-2 \eta_{i}\right)\left\|B_{i} \Lambda_{n}^{i-1} u_{n}-B_{i} p\right\|^{2} \\
& \leq\left\|x_{n}-p\right\|^{2}+\lambda_{i, n}\left(\lambda_{i, n}-2 \eta_{i}\right)\left\|B_{i} \Lambda_{n}^{i-1} u_{n}-B_{i} p\right\|^{2}
\end{aligned}
$$


for $i \in\{1,2, \ldots, N\}$ and $k \in\{1,2, \ldots, M\}$. Combining (3.50), (3.53), and (3.11), we get

$$
\begin{aligned}
\left\|y_{n}-p\right\|^{2} \leq & \beta_{n}\left\|x_{n}-p\right\|^{2}+\left(1-\beta_{n}\right)\left\|v_{n}-p\right\|^{2}-\frac{\beta_{n}}{1-\beta_{n}}\left\|y_{n}-x_{n}\right\|^{2} \\
\leq & \beta_{n}\left\|x_{n}-p\right\|^{2}+\left(1-\beta_{n}\right)\left\|v_{n}-p\right\|^{2} \\
\leq & \beta_{n}\left\|x_{n}-p\right\|^{2}+\left(1-\beta_{n}\right)\left\|\Lambda_{n}^{i} u_{n}-p\right\|^{2} \\
\leq & \beta_{n}\left\|x_{n}-p\right\|^{2}+\left(1-\beta_{n}\right)\left[\left\|u_{n}-p\right\|^{2}+\lambda_{i, n}\left(\lambda_{i, n}-2 \eta_{i}\right)\left\|B_{i} \Lambda_{n}^{i-1} u_{n}-B_{i} p\right\|^{2}\right] \\
\leq & \beta_{n}\left\|x_{n}-p\right\|^{2}+\left(1-\beta_{n}\right)\left[\left\|\Delta_{n}^{k} x_{n}-p\right\|^{2}+\lambda_{i, n}\left(\lambda_{i, n}-2 \eta_{i}\right)\left\|B_{i} \Lambda_{n}^{i-1} u_{n}-B_{i} p\right\|^{2}\right] \\
\leq & \beta_{n}\left\|x_{n}-p\right\|^{2}+\left(1-\beta_{n}\right)\left[\left\|x_{n}-p\right\|^{2}+r_{k, n}\left(r_{k, n}-2 \mu_{k}\right)\left\|A_{k} \Delta_{n}^{k-1} x_{n}-A_{k} p\right\|^{2}\right. \\
& \left.+\lambda_{i, n}\left(\lambda_{i, n}-2 \eta_{i}\right)\left\|B_{i} \Lambda_{n}^{i-1} u_{n}-B_{i} p\right\|^{2}\right] \\
= & \left\|x_{n}-p\right\|^{2}+\left(1-\beta_{n}\right)\left[r_{k, n}\left(r_{k, n}-2 \mu_{k}\right)\left\|A_{k} \Delta_{n}^{k-1} x_{n}-A_{k} p\right\|^{2}\right. \\
& \left.+\lambda_{i, n}\left(\lambda_{i, n}-2 \eta_{i}\right)\left\|B_{i} \Lambda_{n}^{i-1} u_{n}-B_{i} p\right\|^{2}\right],
\end{aligned}
$$

which immediately leads to

$$
\begin{aligned}
& \left(1-\beta_{n}\right)\left[r_{k, n}\left(2 \mu_{k}-r_{k, n}\right)\left\|A_{k} \Delta_{n}^{k-1} x_{n}-A_{k} p\right\|^{2}+\lambda_{i, n}\left(2 \eta_{i}-\lambda_{i, n}\right)\left\|B_{i} \Lambda_{n}^{i-1} u_{n}-B_{i} p\right\|^{2}\right] \\
& \quad \leq\left\|x_{n}-p\right\|^{2}-\left\|y_{n}-p\right\|^{2} \\
& \quad \leq\left\|x_{n}-y_{n}\right\|\left(\left\|x_{n}-p\right\|+\left\|y_{n}-p\right\|\right) .
\end{aligned}
$$

Since $\left\|x_{n}-y_{n}\right\| \rightarrow 0,\left\{\beta_{n}\right\} \subset[a, b] \subset(0,1),\left\{\lambda_{i, n}\right\} \subset\left[a_{i}, b_{i}\right] \subset\left(0,2 \eta_{i}\right),\left\{r_{k, n}\right\} \subset\left[c_{k}, d_{k}\right] \subset$ $\left(0,2 \mu_{k}\right), i \in\{1,2, \ldots, N\}, k \in\{1,2, \ldots, M\}$, and $\left\{x_{n}\right\},\left\{y_{n}\right\}$ are bounded sequences, we have

$$
\lim _{n \rightarrow \infty}\left\|A_{k} \Delta_{n}^{k-1} x_{n}-A_{k} p\right\|=0 \quad \text { and } \quad \lim _{n \rightarrow \infty}\left\|B_{i} \Lambda_{n}^{i-1} u_{n}-B_{i} p\right\|=0
$$

for all $k \in\{1,2, \ldots, M\}$ and $i \in\{1,2, \ldots, N\}$.

Furthermore, by Proposition 2.2(ii) and Lemma 2.2(a), we have

$$
\begin{aligned}
&\left\|\Delta_{n}^{k} x_{n}-p\right\|^{2} \\
&=\left\|T_{r_{k, n}}^{\left(\Theta_{k}, \varphi_{k}\right)}\left(I-r_{k, n} A_{k}\right) \Delta_{n}^{k-1} x_{n}-T_{r_{k, n}}^{\left(\Theta_{k}, \varphi_{k}\right)}\left(I-r_{k, n} A_{k}\right) p\right\|^{2} \\
& \leq\left\langle\left(I-r_{k, n} A_{k}\right) \Delta_{n}^{k-1} x_{n}-\left(I-r_{k, n} A_{k}\right) p, \Delta_{n}^{k} x_{n}-p\right\rangle \\
&= \frac{1}{2}\left(\left\|\left(I-r_{k, n} A_{k}\right) \Delta_{n}^{k-1} x_{n}-\left(I-r_{k, n} A_{k}\right) p\right\|^{2}+\left\|\Delta_{n}^{k} x_{n}-p\right\|^{2}\right. \\
&\left.\quad-\left\|\left(I-r_{k, n} A_{k}\right) \Delta_{n}^{k-1} x_{n}-\left(I-r_{k, n} A_{k}\right) p-\left(\Delta_{n}^{k} x_{n}-p\right)\right\|^{2}\right) \\
& \leq \frac{1}{2}\left(\left\|\Delta_{n}^{k-1} x_{n}-p\right\|^{2}+\left\|\Delta_{n}^{k} x_{n}-p\right\|^{2}-\left\|\Delta_{n}^{k-1} x_{n}-\Delta_{n}^{k} x_{n}-r_{k, n}\left(A_{k} \Delta_{n}^{k-1} x_{n}-A_{k} p\right)\right\|^{2}\right),
\end{aligned}
$$

which implies that

$$
\begin{aligned}
& \left\|\Delta_{n}^{k} x_{n}-p\right\|^{2} \\
& \quad \leq\left\|\Delta_{n}^{k-1} x_{n}-p\right\|^{2}-\left\|\Delta_{n}^{k-1} x_{n}-\Delta_{n}^{k} x_{n}-r_{k, n}\left(A_{k} \Delta_{n}^{k-1} x_{n}-A_{k} p\right)\right\|^{2}
\end{aligned}
$$




$$
\begin{aligned}
= & \left\|\Delta_{n}^{k-1} x_{n}-p\right\|^{2}-\left\|\Delta_{n}^{k-1} x_{n}-\Delta_{n}^{k} x_{n}\right\|^{2}-r_{k, n}^{2}\left\|A_{k} \Delta_{n}^{k-1} x_{n}-A_{k} p\right\|^{2} \\
& +2 r_{k, n}\left|\Delta_{n}^{k-1} x_{n}-\Delta_{n}^{k} x_{n}, A_{k} \Delta_{n}^{k-1} x_{n}-A_{k} p\right\rangle \\
\leq & \left\|\Delta_{n}^{k-1} x_{n}-p\right\|^{2}-\left\|\Delta_{n}^{k-1} x_{n}-\Delta_{n}^{k} x_{n}\right\|^{2} \\
& +2 r_{k, n}\left\|\Delta_{n}^{k-1} x_{n}-\Delta_{n}^{k} x_{n}\right\|\left\|A_{k} \Delta_{n}^{k-1} x_{n}-A_{k} p\right\| \\
\leq & \left\|x_{n}-p\right\|^{2}-\left\|\Delta_{n}^{k-1} x_{n}-\Delta_{n}^{k} x_{n}\right\|^{2} \\
& +2 r_{k, n}\left\|\Delta_{n}^{k-1} x_{n}-\Delta_{n}^{k} x_{n}\right\|\left\|A_{k} \Delta_{n}^{k-1} x_{n}-A_{k} p\right\| .
\end{aligned}
$$

By Lemma 2.2(a) and Lemma 2.10, we obtain

$$
\begin{aligned}
&\left\|\Lambda_{n}^{i} u_{n}-p\right\|^{2} \\
&=\left\|J_{R_{i}, \lambda_{i, n}}\left(I-\lambda_{i, n} B_{i}\right) \Lambda_{n}^{i-1} u_{n}-J_{R_{i}, \lambda_{i, n}}\left(I-\lambda_{i, n} B_{i}\right) p\right\|^{2} \\
& \leq\left\langle\left(I-\lambda_{i, n} B_{i}\right) \Lambda_{n}^{i-1} u_{n}-\left(I-\lambda_{i, n} B_{i}\right) p, \Lambda_{n}^{i} u_{n}-p\right\rangle \\
&= \frac{1}{2}\left(\left\|\left(I-\lambda_{i, n} B_{i}\right) \Lambda_{n}^{i-1} u_{n}-\left(I-\lambda_{i, n} B_{i}\right) p\right\|^{2}+\left\|\Lambda_{n}^{i} u_{n}-p\right\|^{2}\right. \\
&\left.-\left\|\left(I-\lambda_{i, n} B_{i}\right) \Lambda_{n}^{i-1} u_{n}-\left(I-\lambda_{i, n} B_{i}\right) p-\left(\Lambda_{n}^{i} u_{n}-p\right)\right\|^{2}\right) \\
& \leq \frac{1}{2}\left(\left\|\Lambda_{n}^{i-1} u_{n}-p\right\|^{2}+\left\|\Lambda_{n}^{i} u_{n}-p\right\|^{2}-\left\|\Lambda_{n}^{i-1} u_{n}-\Lambda_{n}^{i} u_{n}-\lambda_{i, n}\left(B_{i} \Lambda_{n}^{i-1} u_{n}-B_{i} p\right)\right\|^{2}\right) \\
& \leq \frac{1}{2}\left(\left\|u_{n}-p\right\|^{2}+\left\|\Lambda_{n}^{i} u_{n}-p\right\|^{2}-\left\|\Lambda_{n}^{i-1} u_{n}-\Lambda_{n}^{i} u_{n}-\lambda_{i, n}\left(B_{i} \Lambda_{n}^{i-1} u_{n}-B_{i} p\right)\right\|^{2}\right) \\
& \leq \frac{1}{2}\left(\left\|x_{n}-p\right\|^{2}+\left\|\Lambda_{n}^{i} u_{n}-p\right\|^{2}-\left\|\Lambda_{n}^{i-1} u_{n}-\Lambda_{n}^{i} u_{n}-\lambda_{i, n}\left(B_{i} \Lambda_{n}^{i-1} u_{n}-B_{i} p\right)\right\|^{2}\right),
\end{aligned}
$$

which immediately leads to

$$
\begin{aligned}
&\left\|\Lambda_{n}^{i} u_{n}-p\right\|^{2} \\
& \leq\left\|x_{n}-p\right\|^{2}-\left\|\Lambda_{n}^{i-1} u_{n}-\Lambda_{n}^{i} u_{n}-\lambda_{i, n}\left(B_{i} \Lambda_{n}^{i-1} u_{n}-B_{i} p\right)\right\|^{2} \\
&=\left\|x_{n}-p\right\|^{2}-\left\|\Lambda_{n}^{i-1} u_{n}-\Lambda_{n}^{k} u_{n}\right\|^{2}-\lambda_{i, n}^{2}\left\|B_{i} \Lambda_{n}^{i-1} u_{n}-B_{i} p\right\|^{2} \\
& \quad+2 \lambda_{i, n}\left\langle\Lambda_{n}^{i-1} u_{n}-\Lambda_{n}^{i} u_{n}, B_{i} \Lambda_{n}^{i-1} u_{n}-B_{i} p\right\rangle \\
& \leq\left\|x_{n}-p\right\|^{2}-\left\|\Lambda_{n}^{i-1} u_{n}-\Lambda_{n}^{i} u_{n}\right\|^{2} \\
&+2 \lambda_{i, n}\left\|\Lambda_{n}^{i-1} u_{n}-\Lambda_{n}^{i} u_{n}\right\|\left\|B_{i} \Lambda_{n}^{i-1} u_{n}-B_{i} p\right\| .
\end{aligned}
$$

Combining (3.50) and (3.57), we have

$$
\begin{aligned}
\left\|y_{n}-p\right\|^{2} \leq & \beta_{n}\left\|x_{n}-p\right\|^{2}+\left(1-\beta_{n}\right)\left\|v_{n}-p\right\|^{2}-\frac{\beta_{n}}{1-\beta_{n}}\left\|y_{n}-x_{n}\right\|^{2} \\
\leq & \beta_{n}\left\|x_{n}-p\right\|^{2}+\left(1-\beta_{n}\right)\left\|v_{n}-p\right\|^{2} \\
\leq & \beta_{n}\left\|x_{n}-p\right\|^{2}+\left(1-\beta_{n}\right)\left\|\Lambda_{n}^{i} u_{n}-p\right\|^{2} \\
\leq & \beta_{n}\left\|x_{n}-p\right\|^{2}+\left(1-\beta_{n}\right)\left[\left\|x_{n}-p\right\|^{2}-\left\|\Lambda_{n}^{i-1} u_{n}-\Lambda_{n}^{i} u_{n}\right\|^{2}\right. \\
& \left.+2 \lambda_{i, n}\left\|\Lambda_{n}^{i-1} u_{n}-\Lambda_{n}^{i} u_{n}\right\|\left\|B_{i} \Lambda_{n}^{i-1} u_{n}-B_{i} p\right\|\right]
\end{aligned}
$$




$$
\begin{aligned}
\leq & \left\|x_{n}-p\right\|^{2}-\left(1-\beta_{n}\right)\left\|\Lambda_{n}^{i-1} u_{n}-\Lambda_{n}^{i} u_{n}\right\|^{2} \\
& +2 \lambda_{i, n}\left\|\Lambda_{n}^{i-1} u_{n}-\Lambda_{n}^{i} u_{n}\right\|\left\|B_{i} \Lambda_{n}^{i-1} u_{n}-B_{i} p\right\|,
\end{aligned}
$$

which yields

$$
\begin{aligned}
& \left(1-\beta_{n}\right)\left\|\Lambda_{n}^{i-1} u_{n}-\Lambda_{n}^{i} u_{n}\right\|^{2} \\
& \quad \leq\left\|x_{n}-p\right\|^{2}-\left\|y_{n}-p\right\|^{2}+2 \lambda_{i, n}\left\|\Lambda_{n}^{i-1} u_{n}-\Lambda_{n}^{i} u_{n}\right\|\left\|B_{i} \Lambda_{n}^{i-1} u_{n}-B_{i} p\right\| \\
& \quad \leq\left\|x_{n}-y_{n}\right\|\left(\left\|x_{n}-p\right\|+\left\|y_{n}-p\right\|\right)+2 \lambda_{i, n}\left\|\Lambda_{n}^{i-1} u_{n}-\Lambda_{n}^{i} u_{n}\right\|\left\|B_{i} \Lambda_{n}^{i-1} u_{n}-B_{i} p\right\| .
\end{aligned}
$$

Since $\left\{\beta_{n}\right\} \subset[a, b] \subset(0,1),\left\{\lambda_{i, n}\right\} \subset\left[a_{i}, b_{i}\right] \subset\left(0,2 \eta_{i}\right), i=1,2, \ldots, N$, and $\left\{u_{n}\right\},\left\{x_{n}\right\}$, and $\left\{y_{n}\right\}$ are bounded sequences, we deduce from (3.55) and $\left\|x_{n}-y_{n}\right\| \rightarrow 0$ that

$$
\lim _{n \rightarrow \infty}\left\|\Lambda_{n}^{i-1} u_{n}-\Lambda_{n}^{i} u_{n}\right\|=0, \quad \forall i \in\{1,2, \ldots, N\} .
$$

Also, combining (3.3), (3.57), and (3.20), we deduce that

$$
\begin{aligned}
\left\|y_{n}-p\right\|^{2} \leq & \beta_{n}\left\|x_{n}-p\right\|^{2}+\left(1-\beta_{n}\right)\left\|v_{n}-p\right\|^{2}-\frac{\beta_{n}}{1-\beta_{n}}\left\|y_{n}-x_{n}\right\|^{2} \\
\leq & \beta_{n}\left\|x_{n}-p\right\|^{2}+\left(1-\beta_{n}\right)\left\|v_{n}-p\right\|^{2} \\
\leq & \beta_{n}\left\|x_{n}-p\right\|^{2}+\left(1-\beta_{n}\right)\left\|u_{n}-p\right\|^{2} \\
\leq & \beta_{n}\left\|x_{n}-p\right\|^{2}+\left(1-\beta_{n}\right)\left\|\Delta_{n}^{k} x_{n}-p\right\|^{2} \\
\leq & \beta_{n}\left\|x_{n}-p\right\|^{2}+\left(1-\beta_{n}\right)\left[\left\|x_{n}-p\right\|^{2}-\left\|\Delta_{n}^{k-1} x_{n}-\Delta_{n}^{k} x_{n}\right\|^{2}\right. \\
& \left.+2 r_{k, n}\left\|\Delta_{n}^{k-1} x_{n}-\Delta_{n}^{k} x_{n}\right\|\left\|A_{k} \Delta_{n}^{k-1} x_{n}-A_{k} p\right\|\right] \\
\leq & \left\|x_{n}-p\right\|^{2}-\left(1-\beta_{n}\right)\left\|\Delta_{n}^{k-1} x_{n}-\Delta_{n}^{k} x_{n}\right\|^{2} \\
& +2 r_{k, n}\left\|\Delta_{n}^{k-1} x_{n}-\Delta_{n}^{k} x_{n}\right\|\left\|A_{k} \Delta_{n}^{k-1} x_{n}-A_{k} p\right\|,
\end{aligned}
$$

which yields

$$
\begin{aligned}
& \left(1-\beta_{n}\right)\left\|\Delta_{n}^{k-1} x_{n}-\Delta_{n}^{k} x_{n}\right\|^{2} \\
& \quad \leq\left\|x_{n}-p\right\|^{2}-\left\|y_{n}-p\right\|^{2}+2 r_{k, n}\left\|\Delta_{n}^{k-1} x_{n}-\Delta_{n}^{k} x_{n}\right\|\left\|A_{k} \Delta_{n}^{k-1} x_{n}-A_{k} p\right\| \\
& \quad \leq\left\|x_{n}-y_{n}\right\|\left(\left\|x_{n}-p\right\|+\left\|y_{n}-p\right\|\right)+2 r_{k, n}\left\|\Delta_{n}^{k-1} x_{n}-\Delta_{n}^{k} x_{n}\right\|\left\|A_{k} \Delta_{n}^{k-1} x_{n}-A_{k} p\right\| .
\end{aligned}
$$

Since $\left\{\beta_{n}\right\} \subset[a, b] \subset(0,1),\left\{r_{k, n}\right\} \subset\left[c_{k}, d_{k}\right] \subset\left(0,2 \mu_{k}\right)$ for $k=1,2, \ldots, M$, and $\left\{x_{n}\right\},\left\{y_{n}\right\}$ are bounded sequences, we deduce from (3.55) and $\left\|x_{n}-y_{n}\right\| \rightarrow 0$ that

$$
\lim _{n \rightarrow \infty}\left\|\Delta_{n}^{k-1} x_{n}-\Delta_{n}^{k} x_{n}\right\|=0, \quad \forall k \in\{1,2, \ldots, M\} .
$$

Hence, from (3.58) and (3.16), we get

$$
\begin{aligned}
\left\|x_{n}-u_{n}\right\| & =\left\|\Delta_{n}^{0} x_{n}-\Delta_{n}^{M} x_{n}\right\| \\
& \leq\left\|\Delta_{n}^{0} x_{n}-\Delta_{n}^{1} x_{n}\right\|+\left\|\Delta_{n}^{1} x_{n}-\Delta_{n}^{2} x_{n}\right\|+\cdots+\left\|\Delta_{n}^{M-1} x_{n}-\Delta_{n}^{M} x_{n}\right\| \\
& \rightarrow 0 \quad \text { as } n \rightarrow \infty
\end{aligned}
$$


and

$$
\begin{aligned}
\left\|u_{n}-v_{n}\right\| & =\left\|\Lambda_{n}^{0} u_{n}-\Lambda_{n}^{N} u_{n}\right\| \\
& \leq\left\|\Lambda_{n}^{0} u_{n}-\Lambda_{n}^{1} u_{n}\right\|+\left\|\Lambda_{n}^{1} u_{n}-\Lambda_{n}^{2} u_{n}\right\|+\cdots+\left\|\Lambda_{n}^{N-1} u_{n}-\Lambda_{n}^{N} u_{n}\right\| \\
& \rightarrow 0 \quad \text { as } n \rightarrow \infty
\end{aligned}
$$

respectively. Thus, from (3.60) and (3.18), we obtain

$$
\begin{aligned}
\left\|x_{n}-v_{n}\right\| & \leq\left\|x_{n}-u_{n}\right\|+\left\|u_{n}-v_{n}\right\| \\
& \rightarrow 0 \quad \text { as } n \rightarrow \infty
\end{aligned}
$$

On the other hand, for simplicity, we write $\tilde{p}=P_{C}\left(I-v_{2} F_{2}\right) p, \tilde{v}_{n}=P_{C}\left(I-v_{2} F_{2}\right) v_{n}$, and $k_{n}=G v_{n}=P_{C}\left(I-v_{1} F_{1}\right) \tilde{v}_{n}$ for all $n \geq 1$. Then

$$
p=G p=P_{C}\left(I-v_{1} F_{1}\right) \tilde{p}=P_{C}\left(I-v_{1} F_{1}\right) P_{C}\left(I-v_{2} F_{2}\right) p
$$

We now show that $\lim _{n \rightarrow \infty}\left\|G v_{n}-v_{n}\right\|=0$, that is, $\lim _{n \rightarrow \infty}\left\|k_{n}-v_{n}\right\|=0$. As a matter of fact, for $p \in \Omega$, it follows from (3.4), (3.5), and (3.50) that

$$
\begin{aligned}
\left\|y_{n}-p\right\|^{2} \leq & \beta_{n}\left\|x_{n}-p\right\|^{2}+\left(1-\beta_{n}\right)\left\|G v_{n}-p\right\|^{2}-\frac{\beta_{n}}{1-\beta_{n}}\left\|y_{n}-x_{n}\right\|^{2} \\
\leq & \beta_{n}\left\|x_{n}-p\right\|^{2}+\left(1-\beta_{n}\right)\left\|G v_{n}-p\right\|^{2} \\
= & \beta_{n}\left\|x_{n}-p\right\|^{2}+\left(1-\beta_{n}\right)\left\|k_{n}-p\right\|^{2} \\
\leq & \beta_{n}\left\|x_{n}-p\right\|^{2}+\left(1-\beta_{n}\right)\left[\left\|\tilde{v}_{n}-\tilde{p}\right\|^{2}+v_{1}\left(v_{1}-2 \zeta_{1}\right)\left\|F_{1} \tilde{v}_{n}-F_{1} \tilde{p}\right\|^{2}\right] \\
\leq & \beta_{n}\left\|x_{n}-p\right\|^{2}+\left(1-\beta_{n}\right)\left[\left\|v_{n}-p\right\|^{2}+v_{2}\left(v_{2}-2 \zeta_{2}\right)\left\|F_{2} v_{n}-F_{2} p\right\|^{2}\right. \\
& \left.+v_{1}\left(v_{1}-2 \zeta_{1}\right)\left\|F_{1} \tilde{v}_{n}-F_{1} \tilde{p}\right\|^{2}\right] \\
\leq & \beta_{n}\left\|x_{n}-p\right\|^{2}+\left(1-\beta_{n}\right)\left[\left\|x_{n}-p\right\|^{2}+v_{2}\left(v_{2}-2 \zeta_{2}\right)\left\|F_{2} v_{n}-F_{2} p\right\|^{2}\right. \\
& \left.+v_{1}\left(v_{1}-2 \zeta_{1}\right)\left\|F_{1} \tilde{v}_{n}-F_{1} \tilde{p}\right\|^{2}\right] \\
= & \left\|x_{n}-p\right\|^{2}+\left(1-\beta_{n}\right)\left[v_{2}\left(v_{2}-2 \zeta_{2}\right)\left\|F_{2} v_{n}-F_{2} p\right\|^{2}\right. \\
& \left.+v_{1}\left(v_{1}-2 \zeta_{1}\right)\left\|F_{1} \tilde{v}_{n}-F_{1} \tilde{p}\right\|^{2}\right]
\end{aligned}
$$

which immediately yields

$$
\begin{aligned}
& \left(1-\beta_{n}\right)\left[v_{2}\left(2 \zeta_{2}-v_{2}\right)\left\|F_{2} v_{n}-F_{2} p\right\|^{2}+v_{1}\left(2 \zeta_{1}-v_{1}\right)\left\|F_{1} \tilde{v}_{n}-F_{1} \tilde{p}\right\|^{2}\right] \\
& \quad \leq\left\|x_{n}-p\right\|^{2}-\left\|y_{n}-p\right\|^{2} \\
& \quad \leq\left\|x_{n}-y_{n}\right\|\left(\left\|x_{n}-p\right\|+\left\|y_{n}-p\right\|\right) .
\end{aligned}
$$

Since $\left\|x_{n}-y_{n}\right\| \rightarrow 0,\left\{\beta_{n}\right\} \subset[a, b] \subset(0,1), v_{j} \in\left(0,2 \zeta_{j}\right), j=1,2$, and $\left\{x_{n}\right\},\left\{y_{n}\right\}$ are bounded sequences, we have

$$
\lim _{n \rightarrow \infty}\left\|F_{2} v_{n}-F_{2} p\right\|=0 \text { and } \quad \lim _{n \rightarrow \infty}\left\|F_{1} \tilde{v}_{n}-F_{1} \tilde{p}\right\|=0
$$


Also, in terms of the firm nonexpansivity of $P_{C}$ and the $\zeta_{j}$-inverse-strong monotonicity of $F_{j}$ for $j=1,2$, we obtain from $v_{j} \in\left(0,2 \zeta_{j}\right), j=1,2$, and (3.5)

$$
\begin{aligned}
\left\|\tilde{v}_{n}-\tilde{p}\right\|^{2}= & \left\|P_{C}\left(I-v_{2} F_{2}\right) v_{n}-P_{C}\left(I-v_{2} F_{2}\right) p\right\|^{2} \\
\leq & \left\langle\left(I-v_{2} F_{2}\right) v_{n}-\left(I-v_{2} F_{2}\right) p, \tilde{v}_{n}-\tilde{p}\right\rangle \\
= & \frac{1}{2}\left[\left\|\left(I-v_{2} F_{2}\right) v_{n}-\left(I-v_{2} F_{2}\right) p\right\|^{2}+\left\|\tilde{v}_{n}-\tilde{p}\right\|^{2}\right. \\
& \left.-\left\|\left(I-v_{2} F_{2}\right) v_{n}-\left(I-v_{2} F_{2}\right) p-\left(\tilde{v}_{n}-\tilde{p}\right)\right\|^{2}\right] \\
\leq & \frac{1}{2}\left[\left\|v_{n}-p\right\|^{2}+\left\|\tilde{v}_{n}-\tilde{p}\right\|^{2}-\left\|\left(v_{n}-\tilde{v}_{n}\right)-v_{2}\left(F_{2} v_{n}-F_{2} p\right)-(p-\tilde{p})\right\|^{2}\right] \\
= & \frac{1}{2}\left[\left\|v_{n}-p\right\|^{2}+\left\|\tilde{v}_{n}-\tilde{p}\right\|^{2}-\left\|\left(v_{n}-\tilde{v}_{n}\right)-(p-\tilde{p})\right\|^{2}\right. \\
& \left.+2 v_{2}\left(\left(v_{n}-\tilde{v}_{n}\right)-(p-\tilde{p}), F_{2} v_{n}-F_{2} p\right\rangle-v_{2}^{2}\left\|F_{2} v_{n}-F_{2} p\right\|^{2}\right]
\end{aligned}
$$

and

$$
\begin{aligned}
\left\|k_{n}-p\right\|^{2}= & \left\|P_{C}\left(I-v_{1} F_{1}\right) \tilde{v}_{n}-P_{C}\left(I-v_{1} F_{1}\right) \tilde{p}\right\|^{2} \\
\leq & \left\langle\left(I-v_{1} F_{1}\right) \tilde{v}_{n}-\left(I-v_{1} F_{1}\right) \tilde{p}, k_{n}-p\right\rangle \\
= & \frac{1}{2}\left[\left\|\left(I-v_{1} F_{1}\right) \tilde{v}_{n}-\left(I-v_{1} F_{1}\right) \tilde{p}\right\|^{2}+\left\|k_{n}-p\right\|^{2}\right. \\
& \left.-\left\|\left(I-v_{1} F_{1}\right) \tilde{v}_{n}-\left(I-v_{1} F_{1}\right) \tilde{p}-\left(k_{n}-p\right)\right\|^{2}\right] \\
\leq & \frac{1}{2}\left[\left\|\tilde{v}_{n}-\tilde{p}\right\|^{2}+\left\|k_{n}-p\right\|^{2}-\left\|\left(\tilde{v}_{n}-k_{n}\right)+(p-\tilde{p})\right\|^{2}\right. \\
& \left.+2 v_{1}\left\langle F_{1} \tilde{v}_{n}-F_{1} \tilde{p},\left(\tilde{v}_{n}-k_{n}\right)+(p-\tilde{p})\right\rangle-v_{1}^{2}\left\|F_{1} \tilde{v}_{n}-F_{1} \tilde{p}\right\|^{2}\right] \\
\leq & \frac{1}{2}\left[\left\|v_{n}-p\right\|^{2}+\left\|w_{n}-p\right\|^{2}-\left\|\left(\tilde{v}_{n}-k_{n}\right)+(p-\tilde{p})\right\|^{2}\right. \\
& \left.+2 v_{1}\left\langle F_{1} \tilde{v}_{n}-F_{1} \tilde{p},\left(\tilde{v}_{n}-k_{n}\right)+(p-\tilde{p})\right\rangle\right] .
\end{aligned}
$$

Thus, we have

$$
\begin{aligned}
\left\|\tilde{v}_{n}-\tilde{p}\right\|^{2} \leq & \left\|v_{n}-p\right\|^{2}-\left\|\left(v_{n}-\tilde{v}_{n}\right)-(p-\tilde{p})\right\|^{2} \\
& +2 v_{2}\left(\left(v_{n}-\tilde{v}_{n}\right)-(p-\tilde{p}), F_{2} v_{n}-F_{2} p\right\rangle-v_{2}^{2}\left\|F_{2} v_{n}-F_{2} p\right\|^{2}
\end{aligned}
$$

and

$$
\begin{aligned}
\left\|k_{n}-p\right\|^{2} \leq & \left\|v_{n}-p\right\|^{2}-\left\|\left(\tilde{v}_{n}-k_{n}\right)+(p-\tilde{p})\right\|^{2} \\
& +2 v_{1}\left\|F_{1} \tilde{v}_{n}-F_{1} \tilde{p}\right\|\left\|\left(\tilde{v}_{n}-k_{n}\right)+(p-\tilde{p})\right\| .
\end{aligned}
$$

Consequently, from (3.4), (3.63), and (3.65) it follows that

$$
\begin{aligned}
\left\|y_{n}-p\right\|^{2} & \leq \beta_{n}\left\|x_{n}-p\right\|^{2}+\left(1-\beta_{n}\right)\left[\left\|\tilde{v}_{n}-\tilde{p}\right\|^{2}+v_{1}\left(v_{1}-2 \zeta_{1}\right)\left\|F_{1} \tilde{v}_{n}-F_{1} \tilde{p}\right\|^{2}\right] \\
& \leq \beta_{n}\left\|x_{n}-p\right\|^{2}+\left(1-\beta_{n}\right)\left\|\tilde{v}_{n}-\tilde{p}\right\|^{2}
\end{aligned}
$$




$$
\begin{aligned}
\leq & \beta_{n}\left\|x_{n}-p\right\|^{2}+\left(1-\beta_{n}\right)\left[\left\|v_{n}-p\right\|^{2}-\left\|\left(v_{n}-\tilde{v}_{n}\right)-(p-\tilde{p})\right\|^{2}\right. \\
& \left.+2 v_{2}\left\{\left(v_{n}-\tilde{v}_{n}\right)-(p-\tilde{p}), F_{2} v_{n}-F_{2} p\right\rangle-v_{2}^{2}\left\|F_{2} v_{n}-F_{2} p\right\|^{2}\right] \\
\leq & \beta_{n}\left\|x_{n}-p\right\|^{2}+\left(1-\beta_{n}\right)\left[\left\|x_{n}-p\right\|^{2}-\left\|\left(v_{n}-\tilde{v}_{n}\right)-(p-\tilde{p})\right\|^{2}\right. \\
& \left.+2 v_{2}\left\|\left(v_{n}-\tilde{v}_{n}\right)-(p-\tilde{p})\right\|\left\|F_{2} v_{n}-F_{2} p\right\|\right] \\
\leq & \left\|x_{n}-p\right\|^{2}-\left(1-\beta_{n}\right)\left\|\left(v_{n}-\tilde{v}_{n}\right)-(p-\tilde{p})\right\|^{2} \\
& +2 v_{2}\left\|\left(v_{n}-\tilde{v}_{n}\right)-(p-\tilde{p})\right\|\left\|F_{2} v_{n}-F_{2} p\right\|,
\end{aligned}
$$

which hence leads to

$$
\begin{aligned}
& \left(1-\beta_{n}\right)\left\|\left(v_{n}-\tilde{v}_{n}\right)-(p-\tilde{p})\right\|^{2} \\
& \quad \leq\left\|x_{n}-p\right\|^{2}-\left\|y_{n}-p\right\|^{2}+2 v_{2}\left\|\left(v_{n}-\tilde{v}_{n}\right)-(p-\tilde{p})\right\|\left\|F_{2} v_{n}-F_{2} p\right\| \\
& \quad \leq\left\|x_{n}-y_{n}\right\|\left(\left\|x_{n}-p\right\|+\left\|y_{n}-p\right\|\right)+2 v_{2}\left\|\left(v_{n}-\tilde{v}_{n}\right)-(p-\tilde{p})\right\|\left\|F_{2} v_{n}-F_{2} p\right\| .
\end{aligned}
$$

Since $\left\|x_{n}-y_{n}\right\| \rightarrow 0,\left\{\beta_{n}\right\} \subset[a, b] \subset(0,1), v_{2} \in\left(0,2 \zeta_{2}\right)$, and $\left\{x_{n}\right\},\left\{y_{n}\right\},\left\{v_{n}\right\},\left\{\tilde{v}_{n}\right\}$ are bounded sequences, we obtain from (3.64)

$$
\lim _{n \rightarrow \infty}\left\|\left(v_{n}-\tilde{v}_{n}\right)-(p-\tilde{p})\right\|=0
$$

Furthermore, from (3.4), (3.63), and (3.66), it follows that

$$
\begin{aligned}
\left\|y_{n}-p\right\|^{2} \leq & \beta_{n}\left\|x_{n}-p\right\|^{2}+\left(1-\beta_{n}\right)\left\|k_{n}-p\right\|^{2} \\
\leq & \beta_{n}\left\|x_{n}-p\right\|^{2}+\left(1-\beta_{n}\right)\left[\left\|v_{n}-p\right\|^{2}-\left\|\left(\tilde{v}_{n}-k_{n}\right)+(p-\tilde{p})\right\|^{2}\right. \\
& \left.+2 v_{1}\left\|F_{1} \tilde{v}_{n}-F_{1} \tilde{p}\right\|\left\|\left(\tilde{v}_{n}-k_{n}\right)+(p-\tilde{p})\right\|\right] \\
\leq & \beta_{n}\left\|x_{n}-p\right\|^{2}+\left(1-\beta_{n}\right)\left[\left\|x_{n}-p\right\|^{2}-\left\|\left(\tilde{v}_{n}-k_{n}\right)+(p-\tilde{p})\right\|^{2}\right. \\
& \left.+2 v_{1}\left\|F_{1} \tilde{v}_{n}-F_{1} \tilde{p}\right\|\left\|\left(\tilde{v}_{n}-k_{n}\right)+(p-\tilde{p})\right\|\right] \\
= & \left\|x_{n}-p\right\|^{2}-\left(1-\beta_{n}\right)\left\|\left(\tilde{v}_{n}-k_{n}\right)+(p-\tilde{p})\right\|^{2} \\
& +2 v_{1}\left\|F_{1} \tilde{v}_{n}-F_{1} \tilde{p}\right\|\left\|\left(\tilde{v}_{n}-k_{n}\right)+(p-\tilde{p})\right\|,
\end{aligned}
$$

which hence yields

$$
\begin{aligned}
& \left(1-\beta_{n}\right)\left\|\left(\tilde{v}_{n}-k_{n}\right)+(p-\tilde{p})\right\|^{2} \\
& \quad \leq\left\|x_{n}-p\right\|^{2}-\left\|y_{n}-p\right\|^{2}+2 v_{1}\left\|F_{1} \tilde{v}_{n}-F_{1} \tilde{p}\right\|\left\|\left(\tilde{v}_{n}-k_{n}\right)+(p-\tilde{p})\right\| \\
& \quad \leq\left\|x_{n}-y_{n}\right\|\left(\left\|x_{n}-p\right\|+\left\|y_{n}-p\right\|\right)+2 v_{1}\left\|F_{1} \tilde{v}_{n}-F_{1} \tilde{p}\right\|\left\|\left(\tilde{v}_{n}-k_{n}\right)+(p-\tilde{p})\right\| .
\end{aligned}
$$

Since $\left\|x_{n}-y_{n}\right\| \rightarrow 0,\left\{\beta_{n}\right\} \subset[a, b] \subset(0,1), v_{1} \in\left(0,2 \zeta_{1}\right)$, and $\left\{x_{n}\right\},\left\{y_{n}\right\},\left\{k_{n}\right\},\left\{\tilde{v}_{n}\right\}$ are bounded sequences, we obtain from (3.64)

$$
\lim _{n \rightarrow \infty}\left\|\left(\tilde{v}_{n}-k_{n}\right)+(p-\tilde{p})\right\|=0 .
$$


Note that

$$
\left\|v_{n}-k_{n}\right\| \leq\left\|\left(v_{n}-\tilde{v}_{n}\right)-(p-\tilde{p})\right\|+\left\|\left(\tilde{v}_{n}-k_{n}\right)+(p-\tilde{p})\right\| .
$$

Hence, from (3.67) and (3.68), we get

$$
\lim _{n \rightarrow \infty}\left\|v_{n}-G v_{n}\right\|=\lim _{n \rightarrow \infty}\left\|v_{n}-k_{n}\right\|=0 .
$$

Also, observe that

$$
y_{n}-x_{n}=\gamma_{n}\left(G v_{n}-x_{n}\right)+\delta_{n}\left(T G v_{n}-x_{n}\right), \quad \forall n \geq 0 .
$$

Hence, we find

$$
\begin{aligned}
\delta_{n}\left\|T G v_{n}-v_{n}\right\| & \leq \delta_{n}\left\|T G v_{n}-x_{n}\right\|+\delta_{n}\left\|x_{n}-v_{n}\right\| \\
& =\left\|y_{n}-x_{n}-\gamma_{n}\left(G v_{n}-x_{n}\right)\right\|+\delta_{n}\left\|x_{n}-v_{n}\right\| \\
& \leq\left\|y_{n}-x_{n}\right\|+\gamma_{n}\left\|G v_{n}-x_{n}\right\|+\delta_{n}\left\|x_{n}-v_{n}\right\| \\
& \leq\left\|y_{n}-x_{n}\right\|+\gamma_{n}\left\|G v_{n}-v_{n}\right\|+\gamma_{n}\left\|v_{n}-x_{n}\right\|+\delta_{n}\left\|x_{n}-v_{n}\right\| \\
& =\left\|y_{n}-x_{n}\right\|+\gamma_{n}\left\|G v_{n}-v_{n}\right\|+\left(\gamma_{n}+\delta_{n}\right)\left\|x_{n}-v_{n}\right\| \\
& \leq\left\|y_{n}-x_{n}\right\|+\left\|G v_{n}-v_{n}\right\|+\left\|x_{n}-v_{n}\right\| .
\end{aligned}
$$

So, from $\liminf _{n \rightarrow \infty} \delta_{n}>0$, (3.52), (3.62), and (3.69), it follows that

$$
\lim _{n \rightarrow \infty}\left\|T G v_{n}-v_{n}\right\|=0 .
$$

In addition, noticing that

$$
\begin{aligned}
\left\|T v_{n}-v_{n}\right\| & \leq\left\|T v_{n}-T G v_{n}\right\|+\left\|T G v_{n}-v_{n}\right\| \\
& \leq\left\|v_{n}-G v_{n}\right\|+\left\|T G v_{n}-v_{n}\right\|,
\end{aligned}
$$

we know from (3.69) and (3.70) that

$$
\lim _{n \rightarrow \infty}\left\|T v_{n}-v_{n}\right\|=0
$$

Secondly, we show that $\omega_{w}\left(x_{n}\right) \subset \Omega$.

In fact, since $H$ is reflexive and $\left\{x_{n}\right\}$ is bounded, there exists at least a weak convergence subsequence of $\left\{x_{n}\right\}$. Hence, it is well known that $\omega_{w}\left(x_{n}\right) \neq \emptyset$. Now, take an arbitrary $w \in$ $\omega_{w}\left(x_{n}\right)$. Then there exists a subsequence $\left\{x_{n_{i}}\right\}$ of $\left\{x_{n}\right\}$ such that $x_{n_{i}} \rightarrow w$. From (3.58)-(3.60) and (3.62), we have $u_{n_{i}} \rightarrow w, v_{n_{i}} \rightarrow w, \Lambda_{n_{i}}^{m} u_{n_{i}} \rightarrow w$, and $\Delta_{n_{i}}^{k} x_{n_{i}} \rightarrow w$, where $m \in\{1,2, \ldots, N\}$ and $k \in\{1,2, \ldots, M\}$. Utilizing Lemma 2.3(ii), we deduce from $v_{n_{i}} \rightarrow w$ and (3.71) that $w \in \operatorname{Fix}(T)$. In the meantime, utilizing Lemma 2.5, we obtain from $v_{n_{i}} \rightarrow w$ and (3.69) $w \in \operatorname{GSVI}(G)$.

Next, we prove that $w \in \bigcap_{m=1}^{N} \mathrm{I}\left(B_{m}, R_{m}\right)$. As a matter of fact, since $B_{m}$ is $\eta_{m}$-inversestrongly monotone, $B_{m}$ is a monotone and Lipschitz continuous mapping. It follows from 
Lemma 2.13 that $R_{m}+B_{m}$ is maximal monotone. Let $(v, g) \in G\left(R_{m}+B_{m}\right)$, that is, $g-B_{m} v \in$ $R_{m} v$. Again, since $\Lambda_{n}^{m} u_{n}=J_{R_{m}, \lambda_{m, n}}\left(I-\lambda_{m, n} B_{m}\right) \Lambda_{n}^{m-1} u_{n}, n \geq 1, m \in\{1,2, \ldots, N\}$, we have

$$
\Lambda_{n}^{m-1} u_{n}-\lambda_{m, n} B_{m} \Lambda_{n}^{m-1} u_{n} \in\left(I+\lambda_{m, n} R_{m}\right) \Lambda_{n}^{m} u_{n}
$$

that is,

$$
\frac{1}{\lambda_{m, n}}\left(\Lambda_{n}^{m-1} u_{n}-\Lambda_{n}^{m} u_{n}-\lambda_{m, n} B_{m} \Lambda_{n}^{m-1} u_{n}\right) \in R_{m} \Lambda_{n}^{m} u_{n} .
$$

In terms of the monotonicity of $R_{m}$, we get

$$
\left\langle v-\Lambda_{n}^{m} u_{n}, g-B_{m} v-\frac{1}{\lambda_{m, n}}\left(\Lambda_{n}^{m-1} u_{n}-\Lambda_{n}^{m} u_{n}-\lambda_{m, n} B_{m} \Lambda_{n}^{m-1} u_{n}\right)\right\rangle \geq 0,
$$

and hence,

$$
\begin{aligned}
\langle v & \left.-\Lambda_{n}^{m} u_{n}, g\right\rangle \\
& \geq\left\langle v-\Lambda_{n}^{m} u_{n}, B_{m} v+\frac{1}{\lambda_{m, n}}\left(\Lambda_{n}^{m-1} u_{n}-\Lambda_{n}^{m} u_{n}-\lambda_{m, n} B_{m} \Lambda_{n}^{m-1} u_{n}\right)\right\rangle \\
& =\left\langle v-\Lambda_{n}^{m} u_{n}, B_{m} v-B_{m} \Lambda_{n}^{m} u_{n}+B_{m} \Lambda_{n}^{m} u_{n}-B_{m} \Lambda_{n}^{m-1} u_{n}+\frac{1}{\lambda_{m, n}}\left(\Lambda_{n}^{m-1} u_{n}-\Lambda_{n}^{m} u_{n}\right)\right\rangle \\
& \geq\left\langle v-\Lambda_{n}^{m} u_{n}, B_{m} \Lambda_{n}^{m} u_{n}-B_{m} \Lambda_{n}^{m-1} u_{n}\right\rangle+\left\langle v-\Lambda_{n}^{m} u_{n}, \frac{1}{\lambda_{m, n}}\left(\Lambda_{n}^{m-1} u_{n}-\Lambda_{n}^{m} u_{n}\right)\right\rangle .
\end{aligned}
$$

In particular,

$$
\begin{aligned}
\left\langle v-\Lambda_{n_{i}}^{m} u_{n_{i}}, g\right\rangle \geq & \left\langle v-\Lambda_{n_{i}}^{m} u_{n_{i}}, B_{m} \Lambda_{n_{i}}^{m} u_{n_{i}}-B_{m} \Lambda_{n_{i}}^{m-1} u_{n_{i}}\right\rangle \\
& +\left\langle v-\Lambda_{n_{i}}^{m} u_{n_{i}}, \frac{1}{\lambda_{m, n_{i}}}\left(\Lambda_{n_{i}}^{m-1} u_{n_{i}}-\Lambda_{n_{i}}^{m} u_{n_{i}}\right)\right\rangle .
\end{aligned}
$$

Since $\left\|\Lambda_{n}^{m} u_{n}-\Lambda_{n}^{m-1} u_{n}\right\| \rightarrow 0$ (due to (3.58)) and $\left\|B_{m} \Lambda_{n}^{m} u_{n}-B_{m} \Lambda_{n}^{m-1} u_{n}\right\| \rightarrow 0$ (due to the Lipschitz continuity of $\left.B_{m}\right)$, we conclude from $\Lambda_{n_{i}}^{m} u_{n_{i}} \rightarrow w$ and $\left\{\lambda_{i, n}\right\} \subset\left[a_{i}, b_{i}\right] \subset\left(0,2 \eta_{i}\right)$ that

$$
\lim _{i \rightarrow \infty}\left\langle v-\Lambda_{n_{i}}^{m} u_{n_{i}}, g\right\rangle=\langle v-w, g\rangle \geq 0 .
$$

It follows from the maximal monotonicity of $B_{m}+R_{m}$ that $0 \in\left(R_{m}+B_{m}\right) w$, that is, $w \in$ $\mathrm{I}\left(B_{m}, R_{m}\right)$. Therefore, $w \in \bigcap_{m=1}^{N} \mathrm{I}\left(B_{m}, R_{m}\right)$. Next we prove that $w \in \bigcap_{k=1}^{M} \operatorname{GMEP}\left(\Theta_{k}, \varphi_{k}, A_{k}\right)$. Since $\Delta_{n}^{k} x_{n}=T_{r_{k, n}}^{\left(\Theta_{k}, \varphi_{k}\right)}\left(I-r_{k, n} A_{k}\right) \Delta_{n}^{k-1} x_{n}, n \geq 1, k \in\{1,2, \ldots, M\}$, we have

$$
\begin{aligned}
& \Theta_{k}\left(\Delta_{n}^{k} x_{n}, y\right)+\varphi_{k}(y)-\varphi_{k}\left(\Delta_{n}^{k} x_{n}\right)+\left\langle A_{k} \Delta_{n}^{k-1} x_{n}, y-\Delta_{n}^{k} x_{n}\right\rangle \\
& +\frac{1}{r_{k, n}}\left\langle y-\Delta_{n}^{k} x_{n}, \Delta_{n}^{k} x_{n}-\Delta_{n}^{k-1} x_{n}\right\rangle \geq 0 .
\end{aligned}
$$

By (A2), we have

$$
\varphi_{k}(y)-\varphi_{k}\left(\Delta_{n}^{k} x_{n}\right)+\left\langle A_{k} \Delta_{n}^{k-1} x_{n}, y-\Delta_{n}^{k} x_{n}\right\rangle+\frac{1}{r_{k, n}}\left\langle y-\Delta_{n}^{k} x_{n}, \Delta_{n}^{k} x_{n}-\Delta_{n}^{k-1} x_{n}\right\rangle \geq \Theta_{k}\left(y, \Delta_{n}^{k} x_{n}\right) .
$$


Let $z_{t}=t y+(1-t) w$ for all $t \in(0,1]$ and $y \in C$. This implies that $z_{t} \in C$. Then we have

$$
\begin{aligned}
& \left\langle z_{t}-\Delta_{n}^{k} x_{n}, A_{k} z_{t}\right\rangle \\
& \geq \varphi_{k}\left(\Delta_{n}^{k} x_{n}\right)-\varphi_{k}\left(z_{t}\right)+\left\langle z_{t}-\Delta_{n}^{k} x_{n}, A_{k} z_{t}\right\rangle-\left\langle z_{t}-\Delta_{n}^{k} x_{n}, A_{k} \Delta_{n}^{k-1} x_{n}\right\rangle \\
& \quad-\left\langle z_{t}-\Delta_{n}^{k} x_{n}, \frac{\Delta_{n}^{k} x_{n}-\Delta_{n}^{k-1} x_{n}}{r_{k, n}}\right\rangle+\Theta_{k}\left(z_{t}, \Delta_{n}^{k} x_{n}\right) \\
& =\varphi_{k}\left(\Delta_{n}^{k} x_{n}\right)-\varphi_{k}\left(z_{t}\right)+\left\langle z_{t}-\Delta_{n}^{k} x_{n}, A_{k} z_{t}-A_{k} \Delta_{n}^{k} x_{n}\right\rangle \\
& \quad+\left\langle z_{t}-\Delta_{n}^{k} x_{n}, A_{k} \Delta_{n}^{k} x_{n}-A_{k} \Delta_{n}^{k-1} x_{n}\right\rangle-\left\langle z_{t}-\Delta_{n}^{k} x_{n}, \frac{\Delta_{n}^{k} x_{n}-\Delta_{n}^{k-1} x_{n}}{r_{k, n}}\right\rangle \\
& \quad+\Theta_{k}\left(z_{t}, \Delta_{n}^{k} x_{n}\right) .
\end{aligned}
$$

By (3.59), we have $\left\|A_{k} \Delta_{n}^{k} x_{n}-A_{k} \Delta_{n}^{k-1} x_{n}\right\| \rightarrow 0$ as $n \rightarrow \infty$. Furthermore, by the monotonicity of $A_{k}$, we obtain $\left\langle z_{t}-\Delta_{n}^{k} x_{n}, A_{k} z_{t}-A_{k} \Delta_{n}^{k} x_{n}\right\rangle \geq 0$. Then, by (A4), we get

$$
\left\langle z_{t}-w, A_{k} z_{t}\right\rangle \geq \varphi_{k}(w)-\varphi_{k}\left(z_{t}\right)+\Theta_{k}\left(z_{t}, w\right)
$$

Utilizing (A1), (A4), and (3.73), we obtain

$$
\begin{aligned}
0 & =\Theta_{k}\left(z_{t}, z_{t}\right)+\varphi_{k}\left(z_{t}\right)-\varphi_{k}\left(z_{t}\right) \\
& \leq t \Theta_{k}\left(z_{t}, y\right)+(1-t) \Theta_{k}\left(z_{t}, w\right)+t \varphi_{k}(y)+(1-t) \varphi_{k}(w)-\varphi_{k}\left(z_{t}\right) \\
& \leq t\left[\Theta_{k}\left(z_{t}, y\right)+\varphi_{k}(y)-\varphi_{k}\left(z_{t}\right)\right]+(1-t)\left\langle z_{t}-w, A_{k} z_{t}\right\rangle \\
& =t\left[\Theta_{k}\left(z_{t}, y\right)+\varphi_{k}(y)-\varphi_{k}\left(z_{t}\right)\right]+(1-t) t\left\langle y-w, A_{k} z_{t}\right\rangle
\end{aligned}
$$

and hence

$$
0 \leq \Theta_{k}\left(z_{t}, y\right)+\varphi_{k}(y)-\varphi_{k}\left(z_{t}\right)+(1-t)\left\langle y-w, A_{k} z_{t}\right\rangle
$$

Letting $t \rightarrow 0$, we have, for each $y \in C$,

$$
0 \leq \Theta_{k}(w, y)+\varphi_{k}(y)-\varphi_{k}(w)+\left\langle y-w, A_{k} w\right\rangle
$$

This implies that $w \in \operatorname{GMEP}\left(\Theta_{k}, \varphi_{k}, A_{k}\right)$, and hence, $w \in \bigcap_{k=1}^{M} \operatorname{GMEP}\left(\Theta_{k}, \varphi_{k}, A_{k}\right)$. Thus, $w \in \Omega=\bigcap_{n=1}^{\infty} \operatorname{Fix}\left(T_{n}\right) \cap \bigcap_{k=1}^{M} \operatorname{GMEP}\left(\Theta_{k}, \varphi_{k}, A_{k}\right) \cap \bigcap_{m=1}^{N} \mathrm{I}\left(B_{m}, R_{m}\right)$. Consequently, $w \in$ $\bigcap_{k=1}^{M} \operatorname{GMEP}\left(\Theta_{k}, \varphi_{k}, A_{k}\right) \cap \bigcap_{m=1}^{N} \mathrm{I}\left(B_{m}, R_{m}\right) \cap \operatorname{GSVI}(G) \cap \operatorname{Fix}(T)=: \Omega$. This shows that $\omega_{w}\left(x_{n}\right) \subset \Omega$.

Step 3. We prove that $\omega_{w}\left(x_{n}\right) \subset \Xi$ provided $\left\|x_{n}-x_{n+1}\right\|=o\left(\lambda_{n}\right)$.

Indeed, we first note that $0<\gamma \leq \tau$ and $\mu \eta \geq \tau \Leftrightarrow \kappa \geq \eta$ by (3.41). It is clear that

$$
\langle(\mu F-\gamma S) x-(\mu F-\gamma S) y, x-y\rangle \geq(\mu \eta-\gamma)\|x-y\|^{2}, \quad \forall x, y \in C .
$$

Hence, it follows from $0<\gamma \leq \tau \leq \mu \eta$ that $\mu F-\gamma S$ is monotone. Moreover, it is also obvious that $\mu F-\gamma S$ is $(\mu \kappa+\gamma)$-Lipschitzian. 
Now, take an arbitrary $w \in \omega_{w}\left(x_{n}\right)$. Then there exists a subsequence $\left\{x_{n_{i}}\right\}$ of $\left\{x_{n}\right\}$ such that $x_{n_{i}} \rightarrow w$. Utilizing (3.51), we obtain, for each fixed $p \in \Omega$,

$$
\begin{aligned}
& \left\|x_{n+1}-p\right\|^{2} \\
& \leq\left(1-\lambda_{n} \frac{\tau^{2}-\gamma^{2}}{\tau}\right)\left\|x_{n}-p\right\|^{2}-\frac{\beta_{n}\left(1-\lambda_{n} \tau\right)}{1-\beta_{n}}\left\|y_{n}-x_{n}\right\|^{2} \\
& +2 \lambda_{n} \alpha_{n}\left|(\gamma V p-\mu F p), x_{n+1}-p\right\rangle+2 \lambda_{n}\left(1-\alpha_{n}\right)\left\langle(\gamma S p-\mu F p), x_{n+1}-p\right\rangle \\
& \leq\left\|x_{n}-p\right\|^{2}+2 \lambda_{n} \alpha_{n}\left|(\gamma V-\mu F) p, x_{n+1}-p\right\rangle \\
& +2 \lambda_{n}\left(1-\alpha_{n}\right)\left\langle(\gamma S p-\mu F p), x_{n+1}-p\right\rangle \text {, }
\end{aligned}
$$

which implies that

$$
\begin{aligned}
&\left\langle(\mu F-\gamma S) p, x_{n}-p\right\rangle \\
& \leq\left\langle(\mu F-\gamma S) p, x_{n}-x_{n+1}\right\rangle+\left\langle(\mu F-\gamma S) p, x_{n+1}-p\right\rangle \\
& \leq\|(\mu F-\gamma S) p\|\left\|x_{n}-x_{n+1}\right\|+\frac{\left\|x_{n}-p\right\|^{2}-\left\|x_{n+1}-p\right\|^{2}}{2 \lambda_{n}\left(1-\alpha_{n}\right)} \\
& \quad+\frac{\alpha_{n}}{1-\alpha_{n}}\left\langle(\gamma V-\mu F) p, x_{n+1}-p\right\rangle \\
& \leq\|(\mu F-\gamma S) p\|\left\|x_{n}-x_{n+1}\right\|+\frac{\left\|x_{n}-x_{n+1}\right\|\left(\left\|x_{n}-p\right\|+\left\|x_{n+1}-p\right\|\right)}{2 \lambda_{n}\left(1-\alpha_{n}\right)} \\
&+\frac{\alpha_{n}}{1-\alpha_{n}}\|(\gamma V-\mu F) p\|\left\|x_{n+1}-p\right\| .
\end{aligned}
$$

Since $\alpha_{n} \rightarrow 0$ and $\left\|x_{n}-x_{n+1}\right\|=o\left(\lambda_{n}\right)$, from (3.74), we conclude that

$$
\begin{aligned}
\langle(\mu F-\gamma S) p, w-p\rangle & =\lim _{i \rightarrow \infty}\left\langle(\mu F-\gamma S) p, x_{n_{i}}-p\right\rangle \\
& \leq \limsup _{n \rightarrow \infty}\left\langle(\mu F-\gamma S) p, x_{n}-p\right\rangle \\
& \leq 0, \quad \forall p \in \Omega,
\end{aligned}
$$

that is,

$$
\langle(\mu F-\gamma S) p, w-p\rangle \leq 0, \quad \forall p \in \Omega .
$$

Since $\mu F-\gamma S$ is monotone and $(\mu \kappa+\gamma)$-Lipschitzian, by Minty?s lemma B8], we know that (3.75) is equivalent to the VIP

$$
\langle(\mu F-\gamma S) w, p-w\rangle \geq 0, \quad \forall p \in \Omega
$$

This shows that $w \in \operatorname{VI}(\Omega, \mu F-\gamma S)=: \Xi$, and hence, $\Omega \subset \Xi$.

Step 4. We prove that $\left\{x_{n}\right\}$ converges strongly to a unique solution of Problem 1.2 provided $\left\|x_{n}-x_{n+1}\right\|+\left\|x_{n}-T x_{n}\right\|=o\left(\lambda_{n} \alpha_{n}^{2}\right)$. 
Indeed, it is easy from (3.51) to see that, for each fixed $p \in \Omega$,

$$
\begin{aligned}
\frac{a\left(1-\lambda_{n} \tau\right)}{1-a} \frac{\left\|y_{n}-x_{n}\right\|^{2}}{\alpha_{n}^{2}} \leq & \frac{\beta_{n}\left(1-\lambda_{n} \tau\right)}{1-\beta_{n}} \frac{\left\|y_{n}-x_{n}\right\|^{2}}{\alpha_{n}^{2}} \\
\leq & \frac{\left\|x_{n}-x_{n+1}\right\|}{\alpha_{n}^{2}}\left(\left\|x_{n}-p\right\|+\left\|x_{n+1}-p\right\|\right) \\
& +2 \frac{\lambda_{n}}{\alpha_{n}}\|\gamma V p-\mu F p\|\left\|x_{n+1}-p\right\| \\
& +2 \frac{\lambda_{n}}{\alpha_{n}^{2}}\|\gamma S p-\mu F p\|\left\|x_{n+1}-p\right\|,
\end{aligned}
$$

which, together with $\left\|x_{n}-x_{n+1}\right\|=o\left(\alpha_{n}^{2}\right)$ and $\lambda_{n}=o\left(\alpha_{n}^{2}\right)$, implies that

$$
\lim _{n \rightarrow \infty} \frac{\left\|y_{n}-x_{n}\right\|}{\alpha_{n}}=0
$$

In the meantime, it is clear that

$$
\langle(\mu F-\gamma V) x-(\mu F-\gamma V) y, x-y\rangle \geq(\mu \eta-\gamma \rho)\|x-y\|^{2}, \quad \forall x, y \in C .
$$

Hence, it follows from $0<\gamma \leq \tau \leq \mu \eta$ and $\rho<1$ that $\mu F-\gamma V$ is $(\mu \eta-\gamma \rho)$-strongly monotone. Moreover, it is also obvious that $\mu F-\gamma V$ is $(\mu \kappa+\gamma \rho)$-Lipschitzian. So, we can write $\operatorname{VI}(\mu F-\gamma V, \Xi)=\left\{x^{*}\right\}$. We now take a subsequence $\left\{x_{n_{i}}\right\}$ of $\left\{x_{n}\right\}$ satisfying

$$
\limsup _{n \rightarrow \infty}\left\langle(\gamma V-\mu F) x^{*}, x_{n}-x^{*}\right\rangle=\lim _{i \rightarrow \infty}\left\langle(\gamma V-\mu F) x^{*}, x_{n_{i}}-x^{*}\right\rangle .
$$

Without loss of generality, we may further assume that $x_{n_{i}}-\tilde{x}$; then $\tilde{x} \in \Xi$ as we just proved. Since $x^{*}$ is a solution of the THVIP (1.10), we get

$$
\limsup _{n \rightarrow \infty}\left\langle(\gamma V-\mu F) x^{*}, x_{n}-x^{*}\right\rangle=\left\langle(\gamma V-\mu F) x^{*}, \tilde{x}-x^{*}\right\rangle \leq 0 .
$$

From (3.1), (3.6), and (3.51) with $p=x^{*}$, it follows that

$$
\begin{aligned}
\left\|x_{n+1}-x^{*}\right\|^{2} & \\
\leq & {\left[\lambda_{n}\left(1-\alpha_{n}(1-\rho)\right) \gamma\left\|x_{n}-x^{*}\right\|+\left(1-\lambda_{n} \tau\right)\left\|y_{n}-x^{*}\right\|\right]^{2} } \\
& +2 \lambda_{n} \alpha_{n}\left\langle\left(\gamma V x^{*}-\mu F x^{*}\right), x_{n+1}-x^{*}\right\rangle+2 \lambda_{n}\left(1-\alpha_{n}\right)\left\langle\left(\gamma S x^{*}-\mu F x^{*}\right), x_{n+1}-x^{*}\right\rangle \\
\leq & {\left[\lambda_{n}\left(1-\alpha_{n}(1-\rho)\right) \gamma\left\|x_{n}-x^{*}\right\|+\left(1-\lambda_{n} \tau\right)\left\|x_{n}-x^{*}\right\|\right]^{2} } \\
& +2 \lambda_{n} \alpha_{n}\left\langle\left(\gamma V x^{*}-\mu F x^{*}\right), x_{n+1}-x^{*}\right\rangle+2 \lambda_{n}\left(1-\alpha_{n}\right)\left\langle\left(\gamma S x^{*}-\mu F x^{*}\right), x_{n+1}-x^{*}\right\rangle \\
= & \left.11-\lambda_{n}(\tau-\gamma)-\lambda_{n} \alpha_{n}(1-\rho) \gamma\left\|x_{n}-x^{*}\right\|\right]^{2}+2 \lambda_{n} \alpha_{n}\left\langle\left(\gamma V x^{*}-\mu F x^{*}\right), x_{n+1}-x^{*}\right\rangle \\
& +2 \lambda_{n}\left(1-\alpha_{n}\right)\left\langle\left(\gamma S x^{*}-\mu F x^{*}\right), x_{n+1}-x^{*}\right\rangle \\
\leq & {\left[1-\lambda_{n} \alpha_{n}(1-\rho) \gamma\right]\left\|x_{n}-x^{*}\right\|^{2}+2 \lambda_{n} \alpha_{n}\left\langle\left(\gamma V x^{*}-\mu F x^{*}\right), x_{n+1}-x^{*}\right\rangle } \\
& +2 \lambda_{n}\left(1-\alpha_{n}\right)\left\langle\left(\gamma S x^{*}-\mu F x^{*}\right), x_{n+1}-x^{*}\right\rangle
\end{aligned}
$$




$$
\begin{aligned}
= & {\left[1-\lambda_{n} \alpha_{n}(1-\rho) \gamma\right]\left\|x_{n}-x^{*}\right\|^{2} } \\
& +\lambda_{n} \alpha_{n}(1-\rho) \gamma \cdot \frac{2}{(1-\rho) \gamma}\left[\left\langle\left(\gamma V x^{*}-\mu F x^{*}\right), x_{n+1}-x^{*}\right\rangle\right. \\
& \left.+\frac{1-\alpha_{n}}{\alpha_{n}}\left\langle\left(\gamma S x^{*}-\mu F x^{*}\right), x_{n+1}-x^{*}\right\rangle\right] .
\end{aligned}
$$

So, it follows from $x^{*} \in \Xi$ and condition (iv) that

$$
\begin{aligned}
& \left\|x_{n+1}-x^{*}\right\|^{2} \\
& \leq\left[1-\lambda_{n} \alpha_{n}(1-\rho) \gamma\right]\left\|x_{n}-x^{*}\right\|^{2} \\
& \quad+\lambda_{n} \alpha_{n}(1-\rho) \gamma \cdot \frac{2}{(1-\rho) \gamma}\left[\left\langle\left(\gamma V x^{*}-\mu F x^{*}\right), x_{n+1}-x^{*}\right\rangle\right. \\
& \left.\quad+\frac{1-\alpha_{n}}{\alpha_{n}}\left\|(\gamma S-\mu F) x^{*}\right\|\left(\frac{1}{\bar{k}}\left\|x_{n+1}-T x_{n+1}\right\|\right)\right] .
\end{aligned}
$$

In addition, utilizing Lemma 2.3(i), we have

$$
\begin{aligned}
& \left\|T x_{n+1}-x_{n+1}\right\| \\
& \quad \leq\left\|x_{n+1}-T x_{n}\right\|+\left\|T x_{n}-T x_{n+1}\right\| \\
& \quad \leq \frac{1+\xi}{1-\xi}\left\|x_{n}-x_{n+1}\right\|+\left\|\lambda_{n} \gamma\left(\alpha_{n} V x_{n}+\left(1-\alpha_{n}\right) S x_{n}\right)+\left(I-\lambda_{n} \mu F\right) y_{n}-T x_{n}\right\| \\
& \quad \leq \frac{1+\xi}{1-\xi}\left\|x_{n}-x_{n+1}\right\|+\left\|y_{n}-T x_{n}\right\|+\lambda_{n}\left\|\gamma\left(\alpha_{n} V x_{n}+\left(1-\alpha_{n}\right) S x_{n}\right)-\mu F y_{n}\right\| \\
& \quad \leq \frac{1+\xi}{1-\xi}\left\|x_{n}-x_{n+1}\right\|+\left\|y_{n}-x_{n}\right\|+\left\|x_{n}-T x_{n}\right\|+\lambda_{n}\left\|\gamma \alpha_{n}\left(V x_{n}-S x_{n}\right)+\gamma S x_{n}-\mu F y_{n}\right\| \\
& \quad \leq \frac{1+\xi}{1-\xi}\left\|x_{n}-x_{n+1}\right\|+\left\|y_{n}-x_{n}\right\|+\left\|x_{n}-T x_{n}\right\|+\lambda_{n} \widetilde{M}_{0},
\end{aligned}
$$

where $\sup _{n \geq 0}\left\|\gamma \alpha_{n}\left(V x_{n}-S x_{n}\right)+\gamma S x_{n}-\mu F y_{n}\right\| \leq \widetilde{M}_{0}$ for some $\widetilde{M}_{0}>0$. Hence, for a big enough constant $\bar{k}_{1}>0$, from (3.79), we have

$$
\begin{aligned}
& \left\|x_{n+1}-x^{*}\right\|^{2} \\
& \leq\left[1-\lambda_{n} \alpha_{n}(1-\rho) \gamma\right]\left\|x_{n}-x^{*}\right\|^{2} \\
& \quad+\lambda_{n} \alpha_{n}(1-\rho) \gamma \cdot \frac{2}{(1-\rho) \gamma}\left[\left\langle\left(\gamma V x^{*}-\mu F x^{*}\right), x_{n+1}-x^{*}\right\rangle\right. \\
& \left.\quad+\frac{1-\alpha_{n}}{\bar{k} \alpha_{n}}\left\|(\gamma S-\mu F) x^{*}\right\|\left(\frac{1+\xi}{1-\xi}\left\|x_{n}-x_{n+1}\right\|+\left\|y_{n}-x_{n}\right\|+\left\|x_{n}-T x_{n}\right\|+\lambda_{n} \tilde{M}_{0}\right)\right] \\
& \leq\left[1-\lambda_{n} \alpha_{n}(1-\rho) \gamma\right]\left\|x_{n}-x^{*}\right\|^{2} \\
& \quad+\lambda_{n} \alpha_{n}(1-\rho) \gamma \cdot \frac{2}{(1-\rho) \gamma}\left[\left\langle\left(\gamma V x^{*}-\mu F x^{*}\right), x_{n+1}-x^{*}\right\rangle\right. \\
& \left.\quad+\frac{\bar{k}_{1}}{\alpha_{n}}\left(\lambda_{n}+\left\|x_{n}-x_{n+1}\right\|+\left\|y_{n}-x_{n}\right\|+\left\|x_{n}-T x_{n}\right\|\right)\right]
\end{aligned}
$$


Since $\sum_{n=0}^{\infty} \alpha_{n} \lambda_{n}=\infty, \lim _{n \rightarrow \infty} \frac{\lambda_{n}}{\alpha_{n}^{2}}=0,\left\|x_{n}-x_{n+1}\right\|+\left\|x_{n}-T x_{n}\right\|=o\left(\alpha_{n}\right)$, we deduce from (3.76) and (3.78) that $\sum_{n=0}^{\infty} \alpha_{n} \lambda_{n}(1-\rho) \gamma=\infty$ and

$$
\begin{aligned}
& \limsup _{n \rightarrow \infty} \frac{2}{(1-\rho) \gamma}\left[\left\langle\left(\gamma V x^{*}-\mu F x^{*}\right), x_{n+1}-x^{*}\right\rangle\right. \\
& \left.\quad+\frac{\bar{k}_{1}}{\alpha_{n}}\left(\lambda_{n}+\left\|x_{n}-x_{n+1}\right\|+\left\|y_{n}-x_{n}\right\|+\left\|x_{n}-T x_{n}\right\|\right)\right] \leq 0 .
\end{aligned}
$$

Applying Lemma 2.8 to (3.80), we conclude that $x_{n} \rightarrow x^{*}$ as $n \rightarrow \infty$. This completes the proof.

Remark 3.3 It is obvious that iterative scheme (3.1) is different from the iterative schemes considered in [7, 32]. We extended the three-step iterative scheme in [7, Algorithm I] to our four-step iterative scheme (3.1) for the THVIP (1.10) by combining Korpelevich?s extragradient method, the viscosity approximation method, the hybrid steepest-descent method [41], and Mann?s iteration method. It is worth pointing out that under the lack of assumptions similar to those in [32, Theorem 3.2], for example, $\left\{x_{n}\right\}$ is bounded and Fix $(T) \bigcap$ int $C \neq \emptyset$, the sequence $\left\{x_{n}\right\}$ generated by (3.1) converges strongly to a point $x^{*} \in$ $\bigcap_{k=1}^{M} \operatorname{GMEP}\left(\Theta_{k}, \varphi_{k}, A_{k}\right) \cap \bigcap_{i=1}^{N} \mathrm{I}\left(B_{i}, R_{i}\right) \cap \operatorname{GSVI}(G) \cap \operatorname{Fix}(T)=: \Omega$, which is a unique solution $x^{*} \in \Xi$ of THVIP (1.10) (over the fixed point set of a strictly pseudocontractive mapping $T)$, that is, $\left\langle(\mu F-\gamma V) x^{*}, p-x^{*}\right\rangle \geq 0, \forall p \in \Xi$.

Remark 3.4 Theorem 3.2 improves and extends [32, Theorems 3.1 and 3.2] and [7, Theorem 14] in the following aspects:

(a) THVIP (1.10), with the unique solution $x^{*} \in \Omega$ satisfying

$$
x^{*}=P \bigcap_{k=1}^{M} \operatorname{GMEP}\left(\Theta_{k}, \varphi_{k}, A_{k}\right) \cap \bigcap_{i=1}^{N} \mathrm{I}\left(B_{i}, R_{i}\right) \cap \operatorname{GSVI}(G) \cap \operatorname{Fix}(T)(I-(\mu F-\gamma S)) x^{*},
$$

is more general than the problem of finding a point $\tilde{x} \in C$ satisfying $\tilde{x}=P_{\operatorname{Fix}(T)} S \tilde{x}$ in [32] and than the problem of finding a point $x^{*} \in \operatorname{Fix}(T) \cap \operatorname{VI}(C, A)$ satisfying $x^{*}=P_{\mathrm{Fix}(T) \cap \mathrm{VI}(C, A)}(I-(\mu F-\gamma S)) x^{*}$ in [7, Theorem 14]. It is worth pointing out that $S$ is nonexpansive if and only if the complement $I-S$ is $\frac{1}{2}$-inverse-strongly monotone.

(b) Four-step iterative scheme (3.1) for THVIP (1.10) is flexible, and subtle than those considered in [7, Algorithm I] and [32] because it can be used to solve several kinds of problems, for example, the THVIP, the HVIP and the problem of finding a common point of four sets: $\bigcap_{k=1}^{M} \operatorname{GMEP}\left(\Theta_{k}, \varphi_{k}, A_{k}\right), \bigcap_{i=1}^{N} \mathrm{I}\left(B_{i}, R_{i}\right), \operatorname{GSVI}(G)$, and $\operatorname{Fix}(T)$. In addition, Theorem 3.2 drops the crucial requirements in [32, Theorem 3.2] that $\lim _{n \rightarrow \infty} \frac{\alpha_{n}}{\beta_{n}}=0, \lim _{n \rightarrow \infty} \frac{\beta_{n}^{2}}{\alpha_{n}}=0, \operatorname{Fix}(T) \cap \operatorname{int} C \neq \emptyset$, and $\left\{x_{n}\right\}$ is bounded, and also it removes the crucial ones in [7, Theorem 14] that $\sum_{n=0}^{\infty} \alpha_{n}<\infty$, $\lim _{n \rightarrow \infty}\left(\alpha_{n} / \lambda_{n}^{2}\right)=0$. In the meantime, Problem 1.1 (that is, [7, Problem II]) is extended and generalized to the setting of the GSVI (1.4), finitely many GMEPs, and finitely many variational inclusions in our Problem 1.2.

(c) The argument and technique in Theorem 3.2 are different from [32, Theorems 3.1 and 3.2] and [7, Theorem 14] because we make use of the properties of strictly pseudocontractive mappings (see Lemmas 2.3 and 2.4), the properties of resolvent operators and maximal monotone mappings (see Proposition 2.2, Remark 2.2, and Lemmas 2.9-2.13), the fixed point equation $x^{*}=P_{C}\left(I-v_{1} F_{1}\right) P_{C}\left(I-v_{2} F_{2}\right) x^{*}$ 
equivalent to the GSVI (1.4) (see Proposition 2.3) and the contractive coefficient estimates for the contractions associated with nonexpansive mappings

(see Lemma 2.7).

(d) Compared with the requirement $\left\|x_{n}-x_{n+1}\right\|+\left\|x_{n}-z_{n}\right\|=o\left(\lambda_{n}^{2}\right)$ in [7, Theorem 14], the one $\left\|x_{n}-x_{n+1}\right\|+\left\|x_{n}-T x_{n}\right\|=o\left(\lambda_{n} \alpha_{n}^{2}\right)$ in conclusion (c) of Theorem 3.2 is additionally added because Theorem 3.2 involves a quite complex problem, that is, the THVIP (1.10) (over the fixed point set Fix $(T)$ of a strictly pseudocontractive mapping $T$ ) with constraints of several problems: GSVI (1.4), finitely many GMEPs, and finitely many variational inclusions.

\section{Composite Mann-type viscosity approximation method and convergence results}

In this section, we introduce and analyze a multi-step composite Mann-type viscosity iterative algorithm for finding a solution of the THVIP (1.10) (over the fixed point set of a strictly pseudocontractive mapping) with constraints of several problems: finitely many GMEPs, finitely many variational inclusions and GSVI (1.4) in a real Hilbert space. This algorithm is based on Mann?s iteration method, Korpelevich?s extragradient method, the viscosity approximation method, the hybrid steepest-descent method, and the projection method. We prove the strong convergence of the proposed algorithm to a unique solution of THVIP (1.10) under suitable conditions. In addition, we also consider the application of the proposed algorithm to solving a hierarchical VIP with the same constraints.

Algorithm 4.1 Let $C$ be a nonempty, closed, and convex subset of a real Hilbert space $H$. For each $k \in\{1,2, \ldots, M\}$, let $\Theta_{k}: C \times C \rightarrow \mathbb{R}$ be a bifunction satisfying (A1)-(A4) and $\varphi_{k}: C \rightarrow \mathbb{R} \cup\{+\infty\}$ be a proper lower semicontinuous convex function with restriction (B1) or (B2). For each $k \in\{1,2, \ldots, M\}, i \in\{1,2, \ldots, N\}$, let $R_{i}: C \rightarrow 2^{H}$ be a maximal monotone mapping, and $A_{k}: H \rightarrow H$ and $B_{i}: C \rightarrow H$ be $\mu_{k}$-inverse-strongly monotone and $\eta_{i}$-inverse-strongly monotone, respectively. Let $T: C \rightarrow C$ be a $\xi$-strictly pseudocontractive mapping, $S: C \rightarrow C$ be a nonexpansive mapping and $V: C \rightarrow H$ be a $\rho$-contraction with coefficient $\rho \in[0,1)$. Let $F_{j}: C \rightarrow H$ be $\zeta_{j}$-inverse-strongly monotone for $j=1,2$, and $F: C \rightarrow H$ be $\kappa$-Lipschitzian and $\eta$-strongly monotone with positive constants $\kappa, \eta>0$ such that $0<\mu<\frac{2 \eta}{\kappa^{2}}$ and $0<\gamma \leq \tau$ where $\tau=1-\sqrt{1-\mu\left(2 \eta-\mu \kappa^{2}\right)}$. Assume that the solution set $\Xi$ of HVIP (1.11) is nonempty, where $\Omega:=\bigcap_{k=1}^{M} \operatorname{GMEP}\left(\Theta_{k}, \varphi_{k}, A_{k}\right) \cap \bigcap_{i=1}^{N} \mathrm{I}\left(B_{i}, R_{i}\right) \cap$ $\operatorname{GSVI}(G) \cap \operatorname{Fix}(T)$. Let $\left\{\lambda_{n}\right\},\left\{\sigma_{n}\right\},\left\{\alpha_{n}\right\} \subset(0,1],\left\{\beta_{n}\right\},\left\{\gamma_{n}\right\},\left\{\delta_{n}\right\} \subset[0,1],\left\{\lambda_{i, n}\right\} \subset\left[a_{i}, b_{i}\right] \subset$ $\left(0,2 \eta_{i}\right)$, and $\left\{r_{k, n}\right\} \subset\left[c_{k}, d_{k}\right] \subset\left(0,2 \mu_{k}\right)$, where $i \in\{1,2, \ldots, N\}$ and $k \in\{1,2, \ldots, M\}$. For given arbitrarily $x_{0} \in H$, let $\left\{x_{n}\right\}$ be the sequence generated by

$$
\left\{\begin{array}{l}
u_{n}=T_{r_{M, n}}^{\left(\Theta_{M}, \varphi_{M}\right)}\left(I-r_{M, n} A_{M}\right) T_{r_{M-1, n}}^{\left(\Theta_{M-1}, \varphi_{M-1}\right)}\left(I-r_{M-1, n} A_{M-1}\right) \cdots T_{\left.r_{1, n}, \varphi_{1}\right)}^{\left(\Theta_{1}\right.}\left(I-r_{1, n} A_{1}\right) x_{n}, \\
v_{n}=J_{R_{N}, \lambda_{N, n}}\left(I-\lambda_{N, n} B_{N}\right) J_{R_{N-1}, \lambda_{N-1, n}}\left(I-\lambda_{N-1, n} B_{N-1}\right) \cdots J_{R_{1}, \lambda_{1, n}}\left(I-\lambda_{1, n} B_{1}\right) u_{n}, \\
z_{n}=\left(1-\sigma_{n}\right) x_{n}+\sigma_{n} G v_{n}, \\
y_{n}=\beta_{n} x_{n}+\gamma_{n} z_{n}+\delta_{n} T z_{n}, \\
x_{n+1}=P_{C}\left[\lambda_{n} \gamma\left(\alpha_{n} V x_{n}+\left(1-\alpha_{n}\right) S x_{n}\right)+\left(I-\lambda_{n} \mu F\right) y_{n}\right], \quad \forall n \geq 0,
\end{array}\right.
$$

where $G:=P_{C}\left(I-v_{1} F_{1}\right) P_{C}\left(I-v_{2} F_{2}\right)$ with $v_{j} \in\left(0,2 \zeta_{j}\right)$ for $j=1,2$.

Theorem 4.1 In addition to assumptions of Algorithm 4.1, suppose that

(i) $\lim _{n \rightarrow \infty} \lambda_{n}=0, \lim _{n \rightarrow \infty} \alpha_{n}=0$ and $\sum_{n=0}^{\infty} \lambda_{n} \alpha_{n}=\infty$; 
(ii) $\beta_{n}+\gamma_{n}+\delta_{n}=1$ and $\left(\gamma_{n}+\delta_{n}\right) \xi \leq \gamma_{n}$ for all $n \geq 0$;

(iii) $\liminf _{n \rightarrow \infty} \delta_{n}>0, \liminf _{n \rightarrow \infty} \sigma_{n}>0$ and $\left\{\beta_{n}\right\} \subset[a, b] \subset(0,1)$;

(iv) $\lim _{n \rightarrow \infty}\left(\lambda_{n}^{1 / \theta} / \alpha_{n}\right)=0$ and $\|x-T x\| \geq \bar{k}[d(x, \Omega)]^{\theta}, \forall x \in C$, for some $\bar{k}, \theta>0$.

If $\left\{S x_{n}\right\}$ is bounded, then

(a) $\omega_{w}\left(x_{n}\right) \subset \Omega$ provided $\left\|x_{n}-x_{n+1}\right\| \rightarrow 0(n \rightarrow \infty)$;

(b) $\omega_{w}\left(x_{n}\right) \subset \Xi$ provided $\left\|x_{n}-x_{n+1}\right\|+\left\|x_{n}-y_{n}\right\|=o\left(\lambda_{n}\right)$;

(c) $\left\{x_{n}\right\}$ converges strongly to a unique solution of Problem 1.2 provided $\left\|x_{n}-x_{n+1}\right\|+\left\|x_{n}-y_{n}\right\|+\left\|x_{n}-z_{n}\right\|=o\left(\lambda_{n}\right)$.

Proof Since the solution set $\Xi$ of the HVIP (1.11) is nonempty, as is well known, $\Omega \neq \emptyset$. As in the proof of Theorem 3.1, we put

$$
\Delta_{n}^{k}=T_{r_{k, n}}^{\left(\Theta_{k}, \varphi_{k}\right)}\left(I-r_{k, n} A_{k}\right) T_{r_{k-1, n}}^{\left(\Theta_{k-1}, \varphi_{k-1}\right)}\left(I-r_{k-1, n} A_{k-1}\right) \cdots T_{r_{1, n}}^{\left(\Theta_{1}, \varphi_{1}\right)}\left(I-r_{1, n} A_{1}\right) x_{n}
$$

for all $k \in\{1,2, \ldots, M\}$ and $n \geq 1$,

$$
\Lambda_{n}^{i}=J_{R_{i}, \lambda_{i, n}}\left(I-\lambda_{i, n} B_{i}\right) J_{R_{i-1}, \lambda_{i-1, n}}\left(I-\lambda_{i-1, n} B_{i-1}\right) \cdots J_{R_{1}, \lambda_{1, n}}\left(I-\lambda_{1, n} B_{1}\right)
$$

for all $i \in\{1,2, \ldots, N\}, \Delta_{n}^{0}=I$, and $\Lambda_{n}^{0}=I$, where $I$ is the identity mapping on $H$. Then we have $u_{n}=\Delta_{n}^{M} x_{n}$ and $v_{n}=\Lambda_{n}^{N} u_{n}$.

We divide the rest of the proof into several steps.

Step 1. As in the proof of Theorem 3.1, $\left\{x_{n}\right\}$ is bounded. So are the sequences $\left\{u_{n}\right\},\left\{v_{n}\right\}$, $\left\{y_{n}\right\}$, and $\left\{z_{n}\right\}$.

Step 2. We prove that $\omega_{w}\left(x_{n}\right) \subset \Omega$ provided $\left\|x_{n}-x_{n+1}\right\| \rightarrow 0(n \rightarrow \infty)$.

As in the proof of Step 2 in Theorem 3.2, we have

$$
\begin{aligned}
& \left(1-\beta_{n}\right) \sigma_{n}\left[r_{k, n}\left(2 \mu_{k}-r_{k, n}\right)\left\|A_{k} \Delta_{n}^{k-1} x_{n}-A_{k} p\right\|^{2}+\lambda_{i, n}\left(2 \eta_{i}-\lambda_{i, n}\right)\left\|B_{i} \Lambda_{n}^{i-1} u_{n}-B_{i} p\right\|^{2}\right] \\
& \quad \leq\left\|x_{n}-p\right\|^{2}-\left\|y_{n}-p\right\|^{2} \\
& \quad \leq\left\|x_{n}-y_{n}\right\|\left(\left\|x_{n}-p\right\|+\left\|y_{n}-p\right\|\right) .
\end{aligned}
$$

Since $\liminf _{n \rightarrow \infty} \sigma_{n}>0,\left\|x_{n}-y_{n}\right\| \rightarrow 0,\left\{\beta_{n}\right\} \subset[a, b] \subset(0,1),\left\{\lambda_{i, n}\right\} \subset\left[a_{i}, b_{i}\right] \subset\left(0,2 \eta_{i}\right)$, $\left\{r_{k, n}\right\} \subset\left[c_{k}, d_{k}\right] \subset\left(0,2 \mu_{k}\right), i \in\{1,2, \ldots, N\}, k \in\{1,2, \ldots, M\}$, and $\left\{x_{n}\right\},\left\{y_{n}\right\}$ are bounded sequences, we have

$$
\lim _{n \rightarrow \infty}\left\|A_{k} \Delta_{n}^{k-1} x_{n}-A_{k} p\right\|=0 \quad \text { and } \quad \lim _{n \rightarrow \infty}\left\|B_{i} \Lambda_{n}^{i-1} u_{n}-B_{i} p\right\|=0
$$

for all $k \in\{1,2, \ldots, M\}$ and $i \in\{1,2, \ldots, N\}$.

As in the proof of Step 2 in Theorem 3.2, we have

$$
\begin{aligned}
& \left(1-\beta_{n}\right) \sigma_{n}\left\|\left(\tilde{v}_{n}-k_{n}\right)+(p-\tilde{p})\right\|^{2} \\
& \quad \leq\left\|x_{n}-p\right\|^{2}-\left\|y_{n}-p\right\|^{2}+2 v_{1}\left\|F_{1} \tilde{v}_{n}-F_{1} \tilde{p}\right\|\left\|\left(\tilde{v}_{n}-k_{n}\right)+(p-\tilde{p})\right\| \\
& \quad \leq\left\|x_{n}-y_{n}\right\|\left(\left\|x_{n}-p\right\|+\left\|y_{n}-p\right\|\right)+2 v_{1}\left\|F_{1} \tilde{v}_{n}-F_{1} \tilde{p}\right\|\left\|\left(\tilde{v}_{n}-k_{n}\right)+(p-\tilde{p})\right\| .
\end{aligned}
$$


Since $\liminf _{n \rightarrow \infty} \sigma_{n}>0,\left\|x_{n}-y_{n}\right\| \rightarrow 0,\left\{\beta_{n}\right\} \subset[a, b] \subset(0,1), v_{1} \in\left(0,2 \zeta_{1}\right)$, and $\left\{x_{n}\right\},\left\{y_{n}\right\}$, $\left\{k_{n}\right\},\left\{\tilde{v}_{n}\right\}$ are bounded sequences, we obtain from (3.22)

$$
\lim _{n \rightarrow \infty}\left\|\left(\tilde{v}_{n}-k_{n}\right)+(p-\tilde{p})\right\|=0
$$

Note that

$$
\left\|v_{n}-k_{n}\right\| \leq\left\|\left(v_{n}-\tilde{v}_{n}\right)-(p-\tilde{p})\right\|+\left\|\left(\tilde{v}_{n}-k_{n}\right)+(p-\tilde{p})\right\| .
$$

Hence from (3.67) and (4.3), we get

$$
\lim _{n \rightarrow \infty}\left\|v_{n}-G v_{n}\right\|=\lim _{n \rightarrow \infty}\left\|v_{n}-k_{n}\right\|=0
$$

Also, observe that $z_{n}-x_{n}=\sigma_{n}\left(G v_{n}-x_{n}\right)$ and

$$
y_{n}-x_{n}=\gamma_{n}\left(z_{n}-x_{n}\right)+\delta_{n}\left(T z_{n}-x_{n}\right), \quad \forall n \geq 0 .
$$

Hence we find that

$$
\left\|z_{n}-x_{n}\right\| \leq\left\|G v_{n}-x_{n}\right\| \leq\left\|G v_{n}-v_{n}\right\|+\left\|v_{n}-x_{n}\right\|
$$

and

$$
\begin{aligned}
\delta_{n}\left\|T z_{n}-z_{n}\right\| & \leq \delta_{n}\left\|T z_{n}-x_{n}\right\|+\delta_{n}\left\|x_{n}-z_{n}\right\| \\
& =\left\|y_{n}-x_{n}-\gamma_{n}\left(z_{n}-x_{n}\right)\right\|+\delta_{n}\left\|x_{n}-z_{n}\right\| \\
& \leq\left\|y_{n}-x_{n}\right\|+\gamma_{n}\left\|z_{n}-x_{n}\right\|+\delta_{n}\left\|x_{n}-z_{n}\right\| \\
& =\left\|y_{n}-x_{n}\right\|+\left(\gamma_{n}+\delta_{n}\right)\left\|x_{n}-z_{n}\right\| \\
& =\left\|y_{n}-x_{n}\right\|+\left\|x_{n}-z_{n}\right\| .
\end{aligned}
$$

So, from $\liminf _{n \rightarrow \infty} \delta_{n}>0$, (3.52), (3.62), and (4.4) it follows that

$$
\lim _{n \rightarrow \infty}\left\|x_{n}-z_{n}\right\|=0 \quad \text { and } \quad \lim _{n \rightarrow \infty}\left\|T z_{n}-z_{n}\right\|=0 .
$$

Secondly, let us show that $\omega_{w}\left(x_{n}\right) \subset \Omega$.

In fact, since $H$ is reflexive and $\left\{x_{n}\right\}$ is bounded, there exists at least a weak convergence subsequence of $\left\{x_{n}\right\}$. Hence it is well known that $\omega_{w}\left(x_{n}\right) \neq \emptyset$. Now, take an arbitrary $w \in \omega_{w}\left(x_{n}\right)$. Then there exists a subsequence $\left\{x_{n_{i}}\right\}$ of $\left\{x_{n}\right\}$ such that $x_{n_{i}} \rightarrow w$. From (3.58)-(3.60), (3.62), and (4.5), we have that $u_{n_{i}} \rightarrow w, v_{n_{i}} \rightarrow w, z_{n_{i}} \rightarrow w, \Lambda_{n_{i}}^{m} u_{n_{i}} \rightarrow w$ and $\Delta_{n_{i}}^{k} x_{n_{i}} \rightarrow w$, where $m \in\{1,2, \ldots, N\}$ and $k \in\{1,2, \ldots, M\}$. Utilizing Lemma 2.3(ii), we deduce from $z_{n_{i}} \rightarrow w$ and (4.5) that $w \in \operatorname{Fix}(T)$. In the meantime, utilizing Lemma 2.5, we obtain from $v_{n_{i}} \rightarrow w$ and (4.4) $w \in \operatorname{GSVI}(G)$.

As in the proof of Step 2 in Theorem 3.2, $w \in \bigcap_{m=1}^{N} \mathrm{I}\left(B_{m}, R_{m}\right)$.

Step 3. We prove that $\omega_{w}\left(x_{n}\right) \subset \Xi$ provided $\left\|x_{n}-x_{n+1}\right\|+\left\|x_{n}-y_{n}\right\|=o\left(\lambda_{n}\right)$. 
Indeed, as in the proof of Step 3 in Theorem 3.2, we have $0<\gamma \leq \tau$ and $\mu \eta \geq \tau \Leftrightarrow \kappa \geq \eta$. It is clear that

$$
\langle(\mu F-\gamma S) x-(\mu F-\gamma S) y, x-y\rangle \geq(\mu \eta-\gamma)\|x-y\|^{2}, \quad \forall x, y \in C .
$$

Hence, it follows from $0<\gamma \leq \tau \leq \mu \eta$ that $\mu F-\gamma S$ is monotone. Moreover, it is also obvious that $\mu F-\gamma S$ is $(\mu \kappa+\gamma)$-Lipschitzian. Noticing

$$
x_{n+1}=P_{C} w_{n}-w_{n}+\lambda_{n} \gamma\left(\alpha_{n} V x_{n}+\left(1-\alpha_{n}\right) S x_{n}\right)+\left(I-\lambda_{n} \mu F\right) y_{n},
$$

we obtain

$$
\begin{aligned}
x_{n}-x_{n+1}= & w_{n}-P_{C} w_{n}+\alpha_{n} \lambda_{n}(\mu F-\gamma V) x_{n}+\lambda_{n}\left(1-\alpha_{n}\right)(\mu F-\gamma S) x_{n} \\
& +\left(I-\lambda_{n} \mu F\right) x_{n}-\left(I-\lambda_{n} \mu F\right) y_{n} .
\end{aligned}
$$

Set

$$
e_{n}=\frac{x_{n}-x_{n+1}}{\lambda_{n}\left(1-\alpha_{n}\right)}, \quad \forall n \geq 0
$$

It can easily be seen from (3.31) that

$$
\begin{aligned}
e_{n}= & \frac{w_{n}-P_{C} w_{n}}{\lambda_{n}\left(1-\alpha_{n}\right)}+(\mu F-\gamma S) x_{n}+\frac{\alpha_{n}}{1-\alpha_{n}}(\mu F-\gamma V) x_{n} \\
& +\frac{\left(I-\lambda_{n} \mu F\right) x_{n}-\left(I-\lambda_{n} \mu F\right) y_{n}}{\lambda_{n}\left(1-\alpha_{n}\right)} .
\end{aligned}
$$

This yields, for all $p \in \Omega$ (noticing $\left.x_{n}=P_{C} w_{n-1}\right)$,

$$
\begin{aligned}
\left\langle e_{n}, x_{n}-p\right\rangle & \\
= & \frac{1}{\lambda_{n}\left(1-\alpha_{n}\right)}\left\langle w_{n}-P_{C} w_{n}, P_{C} w_{n-1}-p\right\rangle+\left\langle(\mu F-\gamma S) x_{n}, x_{n}-p\right\rangle \\
& +\frac{\alpha_{n}}{1-\alpha_{n}}\left\langle(\mu F-\gamma V) x_{n}, x_{n}-p\right\rangle \\
& +\frac{1}{\lambda_{n}\left(1-\alpha_{n}\right)}\left\langle\left(I-\lambda_{n} \mu F\right) x_{n}-\left(I-\lambda_{n} \mu F\right) y_{n}, x_{n}-p\right\rangle \\
= & \frac{1}{\lambda_{n}\left(1-\alpha_{n}\right)}\left\langle w_{n}-P_{C} w_{n}, P_{C} w_{n}-p\right\rangle+\frac{1}{\lambda_{n}\left(1-\alpha_{n}\right)}\left\langle w_{n}-P_{C} w_{n}, P_{C} w_{n-1}-P_{C} w_{n}\right\rangle \\
& +\left\langle(\mu F-\gamma S) p, x_{n}-p\right\rangle+\left\langle(\mu F-\gamma S) x_{n}-(\mu F-\gamma S) p, x_{n}-p\right\rangle \\
& +\frac{\alpha_{n}}{1-\alpha_{n}}\left\langle(\mu F-\gamma V) x_{n}, x_{n}-p\right\rangle \\
& +\frac{1}{\lambda_{n}\left(1-\alpha_{n}\right)}\left\langle\left(I-\lambda_{n} \mu F\right) x_{n}-\left(I-\lambda_{n} \mu F\right) y_{n}, x_{n}-p\right\rangle .
\end{aligned}
$$

In (4.9), the first term is nonnegative due to Proposition 2.1(i), and the fourth term is also nonnegative due to the monotonicity of $\mu F-\gamma S$. We, therefore, deduce from (4.9) that 
(noticing again $x_{n+1}=P_{C} w_{n}$ )

$$
\begin{aligned}
\left\langle e_{n}, x_{n}-p\right\rangle & \\
\geq & \frac{1}{\lambda_{n}\left(1-\alpha_{n}\right)}\left\langle w_{n}-P_{C} w_{n}, P_{C} w_{n-1}-P_{C} w_{n}\right\rangle+\left\langle(\mu F-\gamma S) p, x_{n}-p\right\rangle \\
& \quad+\frac{\alpha_{n}}{1-\alpha_{n}}\left\langle(\mu F-\gamma V) x_{n}, x_{n}-p\right\rangle+\frac{1}{\lambda_{n}\left(1-\alpha_{n}\right)}\left\langle\left(I-\lambda_{n} \mu F\right) x_{n}-\left(I-\lambda_{n} \mu F\right) y_{n}, x_{n}-p\right\rangle \\
= & \left\langle w_{n}-P_{C} w_{n}, e_{n}\right\rangle+\left\langle(\mu F-\gamma S) p, x_{n}-p\right\rangle+\frac{\alpha_{n}}{1-\alpha_{n}}\left\langle(\mu F-\gamma V) x_{n}, x_{n}-p\right\rangle \\
& +\frac{1}{\lambda_{n}\left(1-\alpha_{n}\right)}\left\langle\left(I-\lambda_{n} \mu F\right) x_{n}-\left(I-\lambda_{n} \mu F\right) y_{n}, x_{n}-p\right\rangle .
\end{aligned}
$$

Note that

$$
\left\|\left(I-\lambda_{n} \mu F\right) x_{n}-\left(I-\lambda_{n} \mu F\right) y_{n}\right\| \leq\left(1-\lambda_{n} \tau\right)\left\|x_{n}-y_{n}\right\| .
$$

Hence, it follows from $\left\|x_{n}-y_{n}\right\|=o\left(\lambda_{n}\right)$ that

$$
\lim _{n \rightarrow \infty} \frac{\left\|\left(I-\lambda_{n} \mu F\right) x_{n}-\left(I-\lambda_{n} \mu F\right) y_{n}\right\|}{\lambda_{n}}=0 .
$$

Also, since $e_{n} \rightarrow 0$ (due to $\left\|x_{n+1}-x_{n}\right\|=o\left(\lambda_{n}\right)$ ), $\alpha_{n} \rightarrow 0$ and $\left\{x_{n}\right\}$ is bounded by Step 1 which implies that $\left\{w_{n}\right\}$ is bounded, we obtain from (4.10)

$$
\limsup _{n \rightarrow \infty}\left\langle(\mu F-\gamma S) p, x_{n}-p\right\rangle \leq 0, \quad \forall p \in \Omega
$$

This suffices to guarantee that $\omega_{w}\left(x_{n}\right) \subset \Xi$; namely, every weak limit point of $\left\{x_{n}\right\}$ solves the HVIP (1.11). As a matter of fact, if $x_{n_{i}} \rightarrow \tilde{x} \in \omega_{w}\left(x_{n}\right)$ for some subsequence $\left\{x_{n_{i}}\right\}$ of $\left\{x_{n}\right\}$, then we deduce from (3.60) that, for all $p \in \Omega$,

$$
\langle(\mu F-\gamma S) p, \tilde{x}-p\rangle=\lim _{i \rightarrow \infty}\left\langle(\mu F-\gamma S) p, x_{n_{i}}-p\right\rangle \leq \limsup _{n \rightarrow \infty}\left\langle(\mu F-\gamma S) p, x_{n}-p\right\rangle \leq 0,
$$

that is,

$$
\langle(\mu F-\gamma S) p, p-\tilde{x}\rangle \geq 0, \quad \forall p \in \Omega
$$

In addition, note that $\omega_{w}\left(x_{n}\right) \subset \Omega$ by Step 2 . Since $\mu F-\gamma S$ is monotone and Lipschitz continuous and $\Omega$ is nonempty, closed, and convex, by Minty?s lemma $\beta 8$ ] the last inequality is equivalent to (1.11). Consequently, we get $\tilde{x} \in \Xi$.

Step 4. We prove that $\left\{x_{n}\right\}$ converges strongly to a unique solution of Problem 1.2 provided $\left\|x_{n}-x_{n+1}\right\|+\left\|x_{n}-y_{n}\right\|+\left\|x_{n}-z_{n}\right\|=o\left(\lambda_{n}\right)$.

Indeed, it is clear that

$$
\langle(\mu F-\gamma V) x-(\mu F-\gamma V) y, x-y\rangle \geq(\mu \eta-\gamma \rho)\|x-y\|^{2}, \quad \forall x, y \in C .
$$

Hence, it follows from $0<\gamma \leq \tau \leq \mu \eta$ and $\rho<1$ that $\mu F-\gamma V$ is $(\mu \eta-\gamma \rho)$-strongly monotone. Moreover, it is also obvious that $\mu F-\gamma V$ is $(\mu \kappa+\gamma \rho)$-Lipschitzian. So, we 
can write $\operatorname{VI}(\mu F-\gamma V, \Xi)=\left\{x^{*}\right\}$. We now take a subsequence $\left\{x_{n_{i}}\right\}$ of $\left\{x_{n}\right\}$ satisfying

$$
\limsup _{n \rightarrow \infty}\left\langle(\gamma V-\mu F) x^{*}, x_{n}-x^{*}\right\rangle=\lim _{i \rightarrow \infty}\left\langle(\gamma V-\mu F) x^{*}, x_{n_{i}}-x^{*}\right\rangle .
$$

Without loss of generality, we may further assume that $x_{n_{i}} \rightarrow \tilde{x}$; then $\tilde{x} \in \Xi$ as we just proved. Since $x^{*}$ is a solution of the THVIP (1.10), we get

$$
\limsup _{n \rightarrow \infty}\left\langle(\gamma V-\mu F) x^{*}, x_{n}-x^{*}\right\rangle=\left\langle(\gamma V-\mu F) x^{*}, \tilde{x}-x^{*}\right\rangle \leq 0 .
$$

From (4.1) and (3.50), it follows that (noticing that $x_{n+1}=P_{C} w_{n}$ and $0<\gamma \leq \tau$ )

$$
\begin{aligned}
\left\|x_{n+1}-x^{*}\right\|^{2} & \\
= & \left\langle w_{n}-x^{*}, x_{n+1}-x^{*}\right\rangle+\left\langle P_{C} w_{n}-w_{n}, P_{C} w_{n}-x^{*}\right\rangle \\
\leq & \left\langle w_{n}-x^{*}, x_{n+1}-x^{*}\right\rangle \\
= & \left\langle\left(I-\lambda_{n} \mu F\right) y_{n}-\left(I-\lambda_{n} \mu F\right) x^{*}, x_{n+1}-x^{*}\right\rangle+\alpha_{n} \lambda_{n} \gamma\left\langle V x_{n}-V x^{*}, x_{n+1}-x^{*}\right\rangle \\
& +\lambda_{n}\left(1-\alpha_{n}\right) \gamma\left\langle S x_{n}-S x^{*}, x_{n+1}-x^{*}\right\rangle+\alpha_{n} \lambda_{n}\left\langle(\gamma V-\mu F) x^{*}, x_{n+1}-x^{*}\right\rangle \\
& +\lambda_{n}\left(1-\alpha_{n}\right)\left\langle(\gamma S-\mu F) x^{*}, x_{n+1}-x^{*}\right\rangle \\
\leq & \left(1-\lambda_{n} \tau\right)\left\|y_{n}-x^{*}\right\|\left\|x_{n+1}-x^{*}\right\|+\left(\alpha_{n} \lambda_{n} \gamma \rho+\lambda_{n}\left(1-\alpha_{n}\right) \gamma\right)\left\|x_{n}-x^{*}\right\|\left\|x_{n+1}-x^{*}\right\| \\
& +\alpha_{n} \lambda_{n}\left\langle(\gamma V-\mu F) x^{*}, x_{n+1}-x^{*}\right\rangle+\lambda_{n}\left(1-\alpha_{n}\right)\left\langle(\gamma S-\mu F) x^{*}, x_{n+1}-x^{*}\right\rangle \\
\leq & \left(1-\lambda_{n} \tau\right)\left\|x_{n}-x^{*}\right\|\left\|x_{n+1}-x^{*}\right\|+\lambda_{n}\left(1-\alpha_{n}(1-\rho)\right) \gamma\left\|x_{n}-x^{*}\right\|\left\|x_{n+1}-x^{*}\right\| \\
& +\alpha_{n} \lambda_{n}\left\langle(\gamma V-\mu F) x^{*}, x_{n+1}-x^{*}\right\rangle+\lambda_{n}\left(1-\alpha_{n}\right)\left\langle(\gamma S-\mu F) x^{*}, x_{n+1}-x^{*}\right\rangle \\
= & \left(1-\lambda_{n}(\tau-\gamma)-\lambda_{n} \alpha_{n}(1-\rho) \gamma\right)\left\|x_{n}-x^{*}\right\|\left\|x_{n+1}-x^{*}\right\| \\
& +\alpha_{n} \lambda_{n}\left\langle(\gamma V-\mu F) x^{*}, x_{n+1}-x^{*}\right\rangle+\lambda_{n}\left(1-\alpha_{n}\right)\left\langle(\gamma S-\mu F) x^{*}, x_{n+1}-x^{*}\right\rangle \\
\leq & \left(1-\lambda_{n} \alpha_{n}(1-\rho) \gamma\right) \frac{1}{2}\left(\left\|x_{n}-x^{*}\right\|^{2}+\left\|x_{n+1}-x^{*}\right\|^{2}\right) \\
& +\alpha_{n} \lambda_{n}\left\langle(\gamma V-\mu F) x^{*}, x_{n+1}-x^{*}\right\rangle+\lambda_{n}\left(1-\alpha_{n}\right)\left\langle(\gamma S-\mu F) x^{*}, x_{n+1}-x^{*}\right\rangle . \\
&
\end{aligned}
$$

It turns out that

$$
\begin{aligned}
\| x_{n+1} & -x^{*} \|^{2} \\
\leq & \frac{1-\alpha_{n} \lambda_{n} \gamma(1-\rho)}{1+\alpha_{n} \lambda_{n} \gamma(1-\rho)}\left\|x_{n}-x^{*}\right\|^{2} \\
& +\frac{2}{1+\alpha_{n} \lambda_{n} \gamma(1-\rho)}\left[\alpha_{n} \lambda_{n}\left\langle(\gamma V-\mu F) x^{*}, x_{n+1}-x^{*}\right\rangle\right. \\
& \left.+\lambda_{n}\left(1-\alpha_{n}\right)\left\langle(\gamma S-\mu F) x^{*}, x_{n+1}-x^{*}\right\rangle\right] \\
\leq & {\left[1-\alpha_{n} \lambda_{n} \gamma(1-\rho)\right]\left\|x_{n}-x^{*}\right\|^{2} } \\
& +\frac{2}{1+\alpha_{n} \lambda_{n} \gamma(1-\rho)}\left[\alpha_{n} \lambda_{n}\left\langle(\gamma V-\mu F) x^{*}, x_{n+1}-x^{*}\right\rangle\right. \\
& \left.+\lambda_{n}\left(1-\alpha_{n}\right)\left\langle(\gamma S-\mu F) x^{*}, x_{n+1}-x^{*}\right\rangle\right] .
\end{aligned}
$$


However, from $x^{*} \in \Xi$ and condition (iv) we obtain

$$
\begin{aligned}
& \left\langle(\gamma S-\mu F) x^{*}, x_{n+1}-x^{*}\right\rangle \\
& \quad=\left\langle(\gamma S-\mu F) x^{*}, x_{n+1}-P_{\Omega} x_{n+1}\right\rangle+\left\langle(\gamma S-\mu F) x^{*}, P_{\Omega} x_{n+1}-x^{*}\right\rangle \\
& \quad \leq\left\|(\gamma S-\mu F) x^{*}\right\|\left\|x_{n+1}-P_{\Omega} x_{n+1}\right\| \\
& \quad \leq\left\|(\gamma S-\mu F) x^{*}\right\| d\left(x_{n+1}, \Omega\right) \\
& \quad \leq\left\|(\gamma S-\mu F) x^{*}\right\|\left(\frac{1}{\bar{k}}\left\|x_{n+1}-T x_{n+1}\right\|\right)^{1 / \theta} .
\end{aligned}
$$

Since $\left(\gamma_{n}+\delta_{n}\right) \xi \leq \gamma_{n}$, utilizing Lemmas 2.3(i) and 2.4, we get

$$
\begin{aligned}
\frac{\left\|\delta_{n}\left(T x_{n}-x_{n}\right)\right\|}{\lambda_{n}} & =\frac{\left\|\delta_{n}\left(T x_{n}-T z_{n}+T z_{n}-x_{n}\right)\right\|}{\lambda_{n}} \\
& \leq \frac{\left\|T x_{n}-T z_{n}\right\|}{\lambda_{n}}+\frac{\left\|\delta_{n}\left(T z_{n}-x_{n}\right)\right\|}{\lambda_{n}} \\
& \leq \frac{1+\xi}{1-\xi} \frac{\left\|x_{n}-z_{n}\right\|}{\lambda_{n}}+\frac{\left\|\delta_{n}\left(T z_{n}-x_{n}\right)\right\|}{\lambda_{n}} \\
& =\frac{1+\xi}{1-\xi} \frac{\left\|x_{n}-z_{n}\right\|}{\lambda_{n}}+\frac{\left\|y_{n}-x_{n}-\gamma_{n}\left(z_{n}-x_{n}\right)\right\|}{\lambda_{n}} \\
& \leq \frac{1+\xi}{1-\xi} \frac{\left\|x_{n}-z_{n}\right\|}{\lambda_{n}}+\frac{\left\|y_{n}-x_{n}\right\|+\gamma_{n}\left\|z_{n}-x_{n}\right\|}{\lambda_{n}} \\
& \leq\left(1+\frac{1+\xi}{1-\xi}\right) \frac{\left\|x_{n}-z_{n}\right\|}{\lambda_{n}}+\frac{\left\|y_{n}-x_{n}\right\|}{\lambda_{n}} \rightarrow 0 \quad \text { as } n \rightarrow \infty .
\end{aligned}
$$

That is, $\left\|\delta_{n}\left(T x_{n}-x_{n}\right)\right\|=o\left(\lambda_{n}\right)$. Taking into account $\liminf _{n \rightarrow \infty} \delta_{n}>0$, we have $\left\|x_{n}-T x_{n}\right\|=$ $o\left(\lambda_{n}\right)$. Furthermore, utilizing Lemma 2.3(i), we have

$$
\begin{aligned}
\| & T x_{n+1}-x_{n+1} \| \\
& \leq\left\|x_{n+1}-T x_{n}\right\|+\left\|T x_{n}-T x_{n+1}\right\| \\
& \leq \frac{1+\xi}{1-\xi}\left\|x_{n}-x_{n+1}\right\|+\left\|\lambda_{n} \gamma\left(\alpha_{n} V x_{n}+\left(1-\alpha_{n}\right) S x_{n}\right)+\left(I-\lambda_{n} \mu F\right) y_{n}-T x_{n}\right\| \\
& \leq \frac{1+\xi}{1-\xi}\left\|x_{n}-x_{n+1}\right\|+\left\|y_{n}-T x_{n}\right\|+\lambda_{n}\left\|\gamma\left(\alpha_{n} V x_{n}+\left(1-\alpha_{n}\right) S x_{n}\right)-\mu F y_{n}\right\| \\
& \leq \frac{1+\xi}{1-\xi}\left\|x_{n}-x_{n+1}\right\|+\left\|y_{n}-x_{n}\right\|+\left\|x_{n}-T x_{n}\right\|+\lambda_{n}\left\|\gamma \alpha_{n}\left(V x_{n}-S x_{n}\right)+\gamma S x_{n}-\mu F y_{n}\right\| \\
& \leq \frac{1+\xi}{1-\xi}\left\|x_{n}-x_{n+1}\right\|+\left\|y_{n}-x_{n}\right\|+\left\|x_{n}-T x_{n}\right\|+\lambda_{n} \tilde{M}_{0},
\end{aligned}
$$

where $\sup _{n \geq 0}\left\|\gamma \alpha_{n}\left(V x_{n}-S x_{n}\right)+\gamma S x_{n}-\mu F y_{n}\right\| \leq \tilde{M}_{0}$ for some $\tilde{M}_{0}>0$. Hence, for a big enough constant $\bar{k}_{1}>0$, from (4.18), we have

$$
\begin{aligned}
& \left\langle(\gamma S-\mu F) x^{*}, x_{n+1}-x^{*}\right\rangle \\
& \quad \leq\left\|(\gamma S-\mu F) x^{*}\right\|\left(\frac{1}{\bar{k}}\left\|x_{n+1}-T x_{n+1}\right\|\right)^{1 / \theta}
\end{aligned}
$$




$$
\begin{aligned}
& \leq\left\|(\gamma S-\mu F) x^{*}\right\|\left[\frac{1}{\bar{k}}\left(\frac{1+\xi}{1-\xi}\left\|x_{n}-x_{n+1}\right\|+\left\|y_{n}-x_{n}\right\|+\left\|x_{n}-T x_{n}\right\|+\lambda_{n} \tilde{M}_{0}\right)\right]^{1 / \theta} \\
& \leq \bar{k}_{1}\left(\lambda_{n}+\left\|x_{n}-x_{n+1}\right\|+\left\|y_{n}-x_{n}\right\|+\left\|x_{n}-T x_{n}\right\|\right)^{1 / \theta} \\
& \leq \bar{k}_{1} \lambda_{n}^{1 / \theta}\left(1+\frac{\left\|x_{n}-x_{n+1}\right\|+\left\|y_{n}-x_{n}\right\|+\left\|x_{n}-T x_{n}\right\|}{\lambda_{n}}\right)^{1 / \theta}
\end{aligned}
$$

Combining (4.17)-(4.19), we get

$$
\begin{aligned}
\| x_{n+1}- & x^{*} \|^{2} \\
\leq & {\left[1-\alpha_{n} \lambda_{n} \gamma(1-\rho)\right]\left\|x_{n}-x^{*}\right\|^{2}+\frac{2}{1+\alpha_{n} \lambda_{n} \gamma(1-\rho)}\left[\alpha_{n} \lambda_{n}\left\langle(\gamma V-\mu F) x^{*}, x_{n+1}-x^{*}\right\rangle\right.} \\
& \left.+\lambda_{n}\left(1-\alpha_{n}\right)\left\langle(\gamma S-\mu F) x^{*}, x_{n+1}-x^{*}\right\rangle\right] \\
\leq & {\left[1-\alpha_{n} \lambda_{n} \gamma(1-\rho)\right]\left\|x_{n}-x^{*}\right\|^{2}+\frac{2 \alpha_{n} \lambda_{n}}{1+\alpha_{n} \lambda_{n} \gamma(1-\rho)}\left[\left\langle(\gamma V-\mu F) x^{*}, x_{n+1}-x^{*}\right\rangle\right.} \\
& \left.+\frac{\bar{k}_{1} \lambda_{n}^{1 / \theta}}{\alpha_{n}}\left(1+\frac{\left\|x_{n}-x_{n+1}\right\|+\left\|y_{n}-x_{n}\right\|+\left\|x_{n}-T x_{n}\right\|}{\lambda_{n}}\right)^{1 / \theta}\right] \\
= & {\left[1-\alpha_{n} \lambda_{n} \gamma(1-\rho)\right]\left\|x_{n}-x^{*}\right\|^{2}+\alpha_{n} \lambda_{n} \gamma(1-\rho) \frac{2}{\left(1+\alpha_{n} \lambda_{n} \gamma(1-\rho)\right) \gamma(1-\rho)} } \\
& \times\left[\left\langle(\gamma V-\mu F) x^{*}, x_{n+1}-x^{*}\right\rangle\right. \\
& \left.+\frac{\bar{k}_{1} \lambda_{n}^{1 / \theta}}{\alpha_{n}}\left(1+\frac{\left\|x_{n}-x_{n+1}\right\|+\left\|y_{n}-x_{n}\right\|+\left\|x_{n}-T x_{n}\right\|}{\lambda_{n}}\right)^{1 / \theta}\right] .
\end{aligned}
$$

Since $\sum_{n=0}^{\infty} \alpha_{n} \lambda_{n}=\infty, \lim _{n \rightarrow \infty} \frac{\lambda_{n}^{1 / \theta}}{\alpha_{n}}=0,\left\|x_{n}-x_{n+1}\right\|+\left\|y_{n}-x_{n}\right\|+\left\|x_{n}-T x_{n}\right\|=o\left(\lambda_{n}\right)$, we deduce from (4.16) that $\sum_{n=0}^{\infty} \alpha_{n} \lambda_{n} \gamma(1-\rho)=\infty$ and

$$
\begin{aligned}
& \limsup _{n \rightarrow \infty} \frac{2}{\left(1+\alpha_{n} \lambda_{n} \gamma(1-\rho)\right) \gamma(1-\rho)}\left[\left\langle(\gamma V-\mu F) x^{*}, x_{n+1}-x^{*}\right\rangle\right. \\
& \left.+\frac{\bar{k}_{1} \lambda_{n}^{1 / \theta}}{\alpha_{n}}\left(1+\frac{\left\|x_{n}-x_{n+1}\right\|+\left\|y_{n}-x_{n}\right\|+\left\|x_{n}-T x_{n}\right\|}{\lambda_{n}}\right)^{1 / \theta}\right] \leq 0 .
\end{aligned}
$$

Applying Lemma 2.8 to (4.19), we conclude that $x_{n} \rightarrow x^{*}$ as $n \rightarrow \infty$. This completes the proof.

Remark 4.1 It is obvious that iterative scheme (4.1) is different from the iterative schemes in $[7,32]$ The three-step iterative scheme in [7, Algorithm I] is extended to the five-step iterative scheme (4.1) for the THVIP (1.10) by combining Mann?s iteration method, Korpelevich?s extragradient method, the viscosity approximation method, the hybrid steepestdescent method [41], and the projection method. It is worth pointing out that under the lack of the assumptions similar to those in [32, Theorem 3.2], for example, $\left\{x_{n}\right\}$ is bounded and $\operatorname{Fix}(T) \cap \operatorname{int} C \neq \emptyset$, the sequence $\left\{x_{n}\right\}$ generated by (3.1) converges strongly to a point $x^{*} \in \bigcap_{k=1}^{M} \operatorname{GMEP}\left(\Theta_{k}, \varphi_{k}, A_{k}\right) \cap \bigcap_{i=1}^{N} \mathrm{I}\left(B_{i}, R_{i}\right) \cap \operatorname{GSVI}(G) \cap \operatorname{Fix}(T)=: \Omega$, which is a unique solution $x^{*} \in \Xi$ of the THVIP (1.10) (over the fixed point set of a strictly pseudocontractive mapping $T)$, that is, $\left\langle(\mu F-\gamma V) x^{*}, p-x^{*}\right\rangle \geq 0, \forall p \in \Xi$. 
Remark 4.2 Theorem 4.1 improves and extends [32, Theorems 3.1 and 3.2] and [7, Theorem 14] in the following aspects:

(a) THVIP (1.10) with the unique solution $x^{*} \in \Omega$ satisfying

$$
x^{*}=P_{\bigcap_{k=1}^{M} \operatorname{GMEP}\left(\Theta_{k}, \varphi_{k}, A_{k}\right) \cap \bigcap_{i=1}^{N} \mathrm{I}\left(B_{i}, R_{i}\right) \cap \mathrm{GSVI}(G) \cap \mathrm{Fix}(T)}(I-(\mu F-\gamma S)) x^{*}
$$

is more general than the problem of finding a point $\tilde{x} \in C$ satisfying $\tilde{x}=P_{\operatorname{Fix}(T)} S \tilde{x}$ in [32] and the problem of finding a point $x^{*} \in \operatorname{Fix}(T) \cap \operatorname{VI}(C, A)$ satisfying $x^{*}=P_{\mathrm{Fix}(T) \cap \mathrm{VI}(C, A)}(I-(\mu F-\gamma S)) x^{*}$ in [7, Theorem 14].

(b) The five-step iterative scheme (4.1) for THVIP (1.10) is flexible and more advantageous than the three-step iterative scheme in [7, Algorithm I] and the two-step iterative scheme in [32, Theorems 3.1 and 3.2] because it can be used to solve several kinds of problems, for example, the THVIP, the HVIP, and the problem of finding a common point of four sets: $\bigcap_{k=1}^{M} \operatorname{GMEP}\left(\Theta_{k}, \varphi_{k}, A_{k}\right), \bigcap_{i=1}^{N} \mathrm{I}\left(B_{i}, R_{i}\right)$, $\operatorname{GSVI}(G)$, and $\operatorname{Fix}(T)$. In addition, Theorem 4.1 drops the crucial requirements in [32, Theorem 3.2] that $\lim _{n \rightarrow \infty} \frac{\alpha_{n}}{\beta_{n}}=0, \lim _{n \rightarrow \infty} \frac{\beta_{n}^{2}}{\alpha_{n}}=0, \operatorname{Fix}(T) \cap \operatorname{int} C \neq \emptyset$, and $\left\{x_{n}\right\}$ is bounded, and also it removes the crucial ones [7, Theorem 14] that $\sum_{n=0}^{\infty} \alpha_{n}<\infty$, $\lim _{n \rightarrow \infty}\left(\alpha_{n} / \lambda_{n}^{2}\right)=0$, and $\left\|x_{n+1}-x_{n}\right\|+\left\|x_{n}-z_{n}\right\|=o\left(\lambda_{n}^{2}\right)$.

(c) The argument and techniques in Theorem 4.1 are different from the ones in [32, Theorems 3.1 and 3.2] and in [7, Theorem 14] because we make use of the properties of strictly pseudocontractive mappings (see Lemmas 2.3 and 2.4), the properties of resolvent operators and maximal monotone mappings (see Proposition 2.2, Remark 2.2, and Lemmas 2.9-2.13), the fixed point equation $x^{*}=P_{C}\left(I-v_{1} F_{1}\right) P_{C}\left(I-v_{2} F_{2}\right) x^{*}$ equivalent to the GSVI (1.4) (see Proposition 2.3), and the contractive coefficient estimates for the contractions associated with nonexpansive mappings (see Lemma 2.7).

(d) Compared with the requirement $\left\|x_{n}-x_{n+1}\right\|+\left\|x_{n}-z_{n}\right\|=o\left(\lambda_{n}^{2}\right)$ in [7, Theorem 14], the one $\left\|x_{n}-x_{n+1}\right\|+\left\|x_{n}-y_{n}\right\|+\left\|x_{n}-z_{n}\right\|=o\left(\lambda_{n}\right)$ in conclusion (c) of Theorem 4.1 is additionally added because Theorem 4.1 involves a quite complex problem, that is, the THVIP 1.10 (over the fixed point set Fix $(T)$ of a strictly pseudocontractive mapping $T$ ) with constraints of several problems: GSVI (1.4), finitely many GMEPs and finitely many variational inclusions.

Competing interests

The authors declare that they have no competing interests.

Authors? contributions

All authors contributed equally to the writing of this paper. All authors read and approved the final manuscript.

\section{Author details}

'Department of Mathematics, Shanghai Normal University, Shanghai, 200234, China. ${ }^{2}$ Department of Occupational Safety and Health, College of Public Health, China Medical University, Taichung, 40421, Taiwan. ${ }^{3}$ Center for Fundamental Science, Kaohsiung Medical University, Kaohsiung, 807, Taiwan.

\section{Acknowledgements}

In this research, first was partially supported by the National Science Foundation of China (11071169), Innovation Program of Shanghai Municipal Education Commission (09ZZ133) and PhD Program Foundation of Ministry of Education of China (20123127110002). In this research, second and third author were partially supported by a grant from National Science Council of Taiwan. 


\section{References}

1. Glowinski, R: Numerical Methods for Nonlinear Variational Problems. Springer, New York (1984)

2. Takahashi, W: Nonlinear Functional Analysis. Yokohama Publishers, Yokohama (2000)

3. Oden, JT: Quantitative Methods on Nonlinear Mechanics. Prentice Hall, Englewood Cliffs (1986)

4. Lions, JL: Quelques Méthodes de Résolution des Problèmes aux Limites Non Linéaires. Dunod, Paris (1969)

5. Zeidler, E: Nonlinear Functional Analysis and Its Applications. Springer, New York (1985)

6. Korpelevich, GM: The extragradient method for finding saddle points and other problems. Matecon 12, 747-756 (1976)

7. Kong, Z-R, Ceng, L-C, Ansari, QH, Pang, C-T: Multistep hybrid extragradient method for triple hierarchical variational inequalities. Abstr. Appl. Anal. 2013, Article ID 718624 (2013)

8. Zeng, L-C, Yao, J-C: Strong convergence theorem by an extragradient method for fixed point problems and variational inequality problems. Taiwan. J. Math. 10, 1293-1303 (2006)

9. Peng, J-W, Yao, J-C: A new hybrid-extragradient method for generalized mixed equilibrium problems, fixed point problems and variational inequality problems. Taiwan. J. Math. 12, 1401-1432 (2008)

10. Ceng, L-C, Ansari, QH, Schaible, S: Hybrid extragradient-like methods for generalized mixed equilibrium problems, system of generalized equilibrium problems and optimization problems. J. Glob. Optim. 53, $69-96$ (2012)

11. Yao, Y, Liou, Y-C, Kang, SM: Approach to common elements of variational inequality problems and fixed point problems via a relaxed extragradient method. Comput. Math. Appl. 59, 3472-3480 (2010)

12. Ceng, L-C, Ansari, QH, Wong, M-M, Yao, J-C: Mann type hybrid extragradient method for variational inequalities, variational inclusions and fixed point problems. Fixed Point Theory 13(2), 403-422 (2012)

13. Nadezhkina, N, Takahashi, W: Weak convergence theorem by an extragradient method for nonexpansive mappings and monotone mappings. J. Optim. Theory Appl. 128, 191-201 (2006)

14. Ceng, L-C, Yao, J-C: A relaxed extragradient-like method for a generalized mixed equilibrium problem, a general system of generalized equilibria and a fixed point problem. Nonlinear Anal. 72, 1922-1937 (2010)

15. Ceng, L-C, Wong, M-M: Relaxed extragradient method for finding a common element of systems of variational inequalities and fixed point problems. Taiwan. J. Math. 17(2), 701-724 (2013)

16. Ceng, L-C, Wang, C-Y, Yao, J-C: Strong convergence theorems by a relaxed extragradient method for a general system of variational inequalities. Math. Methods Oper. Res. 67(3), 375-390 (2008)

17. Ceng, L-C, Ansari, QH, Yao, J-C: Relaxed extragradient iterative methods for variational inequalities. Appl. Math. Comput. 218, 1112-1123 (2011)

18. Ceng, L-C, Petrusel, A: Relaxed extragradient-like method for general system of generalized mixed equilibria and fixed point problem. Taiwan. J. Math. 16(2), 445-478 (2012)

19. Ceng, L-C, Guu, S-M, Yao, J-C: Hybrid iterative method for finding common solutions of generalized mixed equilibrium and fixed point problems. Fixed Point Theory Appl. 2012, 92 (2012)

20. Ceng, L-C, Hu, H-Y, Wong, M-M: Strong and weak convergence theorems for generalized mixed equilibrium problem with perturbation and fixed point problem of infinitely many nonexpansive mappings. Taiwan. J. Math. 15, 1341-1367 (2011)

21. Ansari, QH, Wong, N-C, Yao, J-C: The existence of nonlinear inequalities. Appl. Math. Lett. 12(5), 89-92 (1999)

22. Gwinner, J: Stability of monotone variational inequalities with various applications. In: Giannessi, F, Maugeri, A (eds.) Variational Inequalities and Network Equilibrium Problems, pp. 123-142. Plenum, New York (1995)

23. Takahashi, S, Takahashi, W: Strong convergence theorem for a generalized equilibrium problem and a nonexpansive mapping in a Hilbert space. Nonlinear Anal. 69, 1025-1033 (2008)

24. Ceng, L-C, Yao, J-C: A hybrid iterative scheme for mixed equilibrium problems and fixed point problems. J. Comput. Appl. Math. 214, 186-201 (2008)

25. Rockafellar, RT: Monotone operators and the proximal point algorithms. SIAM J. Control Optim. 14, 877-898 (1976)

26. Huang, N-J: A new completely general class of variational inclusions with noncompact valued mappings. Comput. Math. Appl. 35(10), 9-14 (1998)

27. Zeng, L-C, Guu, S-M, Yao, J-C: Characterization of $\mathrm{H}$-monotone operators with applications to variational inclusions. Comput. Math. Appl. 50, 329-337 (2005)

28. Ceng, L-C, Yao, J-C: On the triple hierarchical variational inequalities with constraints of mixed equilibria, variational inclusions and systems of generalized equilibria. Tamkang J. Math. 45(3), 297-334 (2014). doi:10.5556/j.tkjm.45.2014.1658

29. Zhang, S-S, Lee, HWJ, Chan, CK: Algorithms of common solutions for quasi-variational inclusions and fixed point problems. Appl. Math. Mech. 29, 571-581 (2008)

30. Ceng, L-C, Guu, S-M, Yao, J-C: Hybrid viscosity CQ method for finding a common solution of a variational inequality, a general system of variational inequalities, and a fixed point problem. Fixed Point Theory Appl. 2013, 313 (2013)

31. Verma, RU: On a new system of nonlinear variational inequalities and associated iterative algorithms. Math. Sci. Res. Hot-Line 3(8), 65-68 (1999)

32. Yao, Y, Liou, Y-C, Marino, G: Two-step iterative algorithms for hierarchical fixed point problems and variational inequality problems. J. Appl. Math. Comput. 31, 433-445 (2009)

33. Ceng, L-C, Ansari, QH, Yao, J-C: Iterative methods for triple hierarchical variational inequalities in Hilbert spaces. J. Optim. Theory Appl. 151(3), 489-512 (2011)

34. Ansari, QH, Ceng, L-C, Gupta, H: Triple hierarchical variational inequalities. In: Ansari, QH (ed.) Nonlinear Analysis: Approximation Theory, Optimization and Applications, pp. 231-280. Springer, New York (2014)

35. Ceng, L-C, Ansari, QH, Wen, C-F: Hybrid steepest-descent viscosity method for triple hierarchical variational inequalities. Abstr. Appl. Anal. 2012, Article ID 907105 (2012)

36. Ceng, L-C, Ansari, QH, Yao, J-C: Relaxed hybrid steepest-descent methods with variable parameters for triple-hierarchical variational inequalities. Appl. Anal. 91(10), 1793-1810 (2012)

37. Marino, G, Xu, H-K: Weak and strong convergence theorems for strict pseudo-contractions in Hilbert spaces. J. Math. Anal. Appl. 329, 336-346 (2007)

38. Goebel, K, Kirk, WA: Topics on Metric Fixed-Point Theory. Cambridge University Press, Cambridge (1990)

39. $\mathrm{Xu}, \mathrm{H}-\mathrm{K}, \mathrm{Kim}, \mathrm{T}-\mathrm{H}$ : Convergence of hybrid steepest-descent methods for variational inequalities. J. Optim. Theory Appl. 119, 185-201 (2003) 
40. Barbu, V: Nonlinear Semigroups and Differential Equations in Banach Spaces. Noordhoff, Groningen (1976)

41. Yamada, I: The hybrid steepest-descent method for the variational inequality problems over the intersection of the fixed-point sets of nonexpansive mappings. In: Batnariu, D, Censor, Y, Reich, S (eds.) Inherently Parallel Algorithms in Feasibility and Optimization and Their Applications, pp. 473-504. North-Holland, Amsterdam (2001)

Submit your manuscript to a SpringerOpen ${ }^{\circ}$ journal and benefit from:

- Convenient online submission

Rigorous peer review

- Immediate publication on acceptance

- Open access: articles freely available online

- High visibility within the field

- Retaining the copyright to your article 